Math. Model. Nat. Phenom.

Vol. 5, No. 4, 2010, pp. 390-447

DOI: $10.1051 / \mathrm{mmnp} / 20105416$

\title{
Homogenization of a Periodic Parabolic Cauchy Problem in the Sobolev Space $H^{1}\left(\mathbb{R}^{d}\right)$
}

\author{
T. Suslina* ${ }^{\dagger}$ \\ Department of Physics, St. Petersburg State University, \\ Ul'yanovskaya 3, Petrodvorets, St. Petersburg, 198504, Russia
}

To the memory of my dear Teacher Mikhail Shlemovich Birman

\begin{abstract}
In $L_{2}\left(\mathbb{R}^{d} ; \mathbb{C}^{n}\right)$, we consider a wide class of matrix elliptic second order differential operators $\mathcal{A}_{\varepsilon}$ with rapidly oscillating coefficients (depending on $\mathrm{x} / \varepsilon$ ). For a fixed $\tau>0$ and small $\varepsilon>0$, we find approximation of the operator $\operatorname{exponential~} \exp \left(-\mathcal{A}_{\varepsilon} \tau\right)$ in the $\left(L_{2}\left(\mathbb{R}^{d} ; \mathbb{C}^{n}\right) \rightarrow H^{1}\left(\mathbb{R}^{d} ; \mathbb{C}^{n}\right)\right)$-operator norm with an error term of order $\varepsilon$. In this approximation, the corrector is taken into account. The results are applied to homogenization of a periodic parabolic Cauchy problem.
\end{abstract}

Key words: periodic differential operators, parabolic Cauchy problem, homogenization, effective operator, corrector

AMS subject classification: $35 \mathrm{~B} 27$

\section{Introduction}

The paper is devoted to some problems of homogenization theory for periodic differential operators (DO's). In homogenization theory, the behavior of the solutions of differential equations with rapidly oscillating coefficients in the small period limit is studied. It turns

\footnotetext{
*E-mail: suslina@list.ru

${ }^{\dagger}$ Supported by RFBR (grant no. 08-01-00209-a).
} 
out that approximately the solution of such an equation behaves like the solution of the "homogenized" equation with constant effective coefficients. The character of convergence and error estimates are studied. A broad literature is devoted to homogenization theory. First of all, we mention the books [BeLP], [Sa], [BaPa], [ZhKO].

In [BSu1-5], a new operator-theoretic approach to homogenization problems was suggested and developed. The main idea of this approach is that the homogenization procedure can be studied as a spectral threshold effect. By this approach, the results of a new type in homogenization theory (the so called "operator error estimates") have been obtained. We start with a survey of these results.

\subsection{The class of operators}

We consider matrix second order DO's acting in $L_{2}\left(\mathbb{R}^{d} ; \mathbb{C}^{n}\right)$ and admitting a factorization of the form

$$
\mathcal{A}=f(\mathbf{x})^{*} b(\mathbf{D})^{*} g(\mathbf{x}) b(\mathbf{D}) f(\mathbf{x}) .
$$

Here $f(\mathbf{x})$ is an $(n \times n)$-matrix-valued function and $g(\mathbf{x})$ is an $(m \times m)$-matrix-valued function such that $f, f^{-1} \in L_{\infty}, g, g^{-1} \in L_{\infty}$, and $g(\mathbf{x})$ is positive definite. The coefficients $f(\mathbf{x})$ and $g(\mathbf{x})$ are assumed to be periodic with respect to some lattice $\Gamma \subset \mathbb{R}^{d}$. By $\Omega$ we denote the cell of the lattice $\Gamma$. Next, $b(\mathbf{D})$ is an $(m \times n)$-matrix homogeneous first order DO. It is assumed that $m \geq n$, and that the symbol $b(\boldsymbol{\xi})$ is a matrix of rank $n$ for $0 \neq \boldsymbol{\xi} \in \mathbb{R}^{d}$. In the case $f=\mathbf{1}_{n}$, we use the notation

$$
\widehat{\mathcal{A}}=b(\mathbf{D})^{*} g(\mathbf{x}) b(\mathbf{D})
$$

Many operators of mathematical physics admit a factorization of the form (1.2) or (1.1). The simplest example is the acoustics operator $\widehat{\mathcal{A}}=-\operatorname{div} g(\mathbf{x}) \nabla=\mathbf{D}^{*} g(\mathbf{x}) \mathbf{D}$.

Let $\varepsilon>0$. We denote $\phi^{\varepsilon}(\mathbf{x})=\phi\left(\varepsilon^{-1} \mathbf{x}\right)$ for any $\Gamma$-periodic function $\phi$. The operators

$$
\widehat{\mathcal{A}}_{\varepsilon}=b(\mathbf{D})^{*} g^{\varepsilon}(\mathbf{x}) b(\mathbf{D}), \quad \mathcal{A}_{\varepsilon}=\left(f^{\varepsilon}(\mathbf{x})\right)^{*} b(\mathbf{D})^{*} g^{\varepsilon}(\mathbf{x}) b(\mathbf{D}) f^{\varepsilon}(\mathbf{x}),
$$

are our main objects. The coefficients of operators (1.3) oscillate rapidly as $\varepsilon \rightarrow 0$.

\subsection{Elliptic homogenization problems}

A typical elliptic homogenization problem is to study the behavior of the solution $\mathbf{u}_{\varepsilon}$ of the equation $\widehat{\mathcal{A}}_{\varepsilon} \mathbf{u}_{\varepsilon}+\mathbf{u}_{\varepsilon}=\mathbf{F}$, where $\mathbf{F} \in L_{2}\left(\mathbb{R}^{d} ; \mathbb{C}^{n}\right)$. As $\varepsilon \rightarrow 0$, the solution $\mathbf{u}_{\varepsilon}$ converges (in some sense) to the solution $\mathbf{u}_{0}$ of the "homogenized" equation $\widehat{\mathcal{A}}^{0} \mathbf{u}_{0}+\mathbf{u}_{0}=\mathbf{F}$. Here

$$
\widehat{\mathcal{A}}^{0}=b(\mathbf{D})^{*} g^{0} b(\mathbf{D})
$$

is the so called effective operator, and $g^{0}$ is a constant positive effective matrix. To define $g^{0}$, we introduce a $\Gamma$-periodic $(n \times m)$-matrix-valued function $\Lambda(\mathbf{x})$ which is the solution of the problem

$$
b(\mathbf{D})^{*} g(\mathbf{x})\left(b(\mathbf{D}) \Lambda(\mathbf{x})+\mathbf{1}_{m}\right)=0, \quad \int_{\Omega} \Lambda(\mathbf{x}) d \mathbf{x}=0 .
$$


Then $g^{0}$ is given by $g^{0}=|\Omega|^{-1} \int_{\Omega} g(\mathbf{x})\left(b(\mathbf{D}) \Lambda(\mathbf{x})+\mathbf{1}_{m}\right) d \mathbf{x}$.

In $[\mathrm{BSu} 1,2]$, it was proved that

$$
\left\|\mathbf{u}_{\varepsilon}-\mathbf{u}_{0}\right\|_{L_{2}\left(\mathbb{R}^{d}\right)} \leq C \varepsilon\|\mathbf{F}\|_{L_{2}\left(\mathbb{R}^{d}\right)} .
$$

Estimate (1.5) is order-sharp, and the constant $C$ is controlled explicitly. Inequality (1.5) means that, as $\varepsilon \rightarrow 0$, the resolvent $\left(\widehat{\mathcal{A}}_{\varepsilon}+I\right)^{-1}$ converges to the resolvent of the effective operator in the operator norm in $L_{2}\left(\mathbb{R}^{d} ; \mathbb{C}^{n}\right)$, and

$$
\left\|\left(\widehat{\mathcal{A}}_{\varepsilon}+I\right)^{-1}-\left(\widehat{\mathcal{A}}^{0}+I\right)^{-1}\right\|_{L_{2}\left(\mathbb{R}^{d}\right) \rightarrow L_{2}\left(\mathbb{R}^{d}\right)} \leq C \varepsilon .
$$

In $[\mathrm{BSu} 3,4]$, more accurate approximation of the resolvent $\left(\widehat{\mathcal{A}}_{\varepsilon}+I\right)^{-1}$ in the operator norm in $L_{2}\left(\mathbb{R}^{d} ; \mathbb{C}^{n}\right)$ with an error estimate of order $\varepsilon^{2}$ was obtained:

$$
\left\|\left(\widehat{\mathcal{A}}_{\varepsilon}+I\right)^{-1}-\left(\widehat{\mathcal{A}}^{0}+I\right)^{-1}-\varepsilon K(\varepsilon)\right\|_{L_{2}\left(\mathbb{R}^{d}\right) \rightarrow L_{2}\left(\mathbb{R}^{d}\right)} \leq C \varepsilon^{2} .
$$

In (1.7), the corrector $K(\varepsilon)$ is taken into account; $K(\varepsilon)$ is the sum of three terms:

$$
K(\varepsilon)=K_{1}(\varepsilon)+\left(K_{1}(\varepsilon)\right)^{*}+K_{3} .
$$

The first term $K_{1}(\varepsilon)$ corresponds to the traditional corrector used in homogenization theory. It is given by $K_{1}(\varepsilon)=\Lambda^{\varepsilon} b(\mathbf{D})\left(\widehat{\mathcal{A}}^{0}+I\right)^{-1} \Pi_{\varepsilon}$, where $\Pi_{\varepsilon}$ is an auxiliary smoothing operator (see (11.1) below). In some cases it is possible to "eliminate" $\Pi_{\varepsilon}$ and to replace $K_{1}(\varepsilon)$ by $\Lambda^{\varepsilon} b(\mathbf{D})\left(\widehat{\mathcal{A}}^{0}+I\right)^{-1}$. The third term $K_{3}$ does not depend on $\varepsilon$.

In [BSu5], the resolvent $\left(\widehat{\mathcal{A}}_{\varepsilon}+I\right)^{-1}$ was approximated in the norm of operators acting from $L_{2}\left(\mathbb{R}^{d} ; \mathbb{C}^{n}\right)$ to $H^{1}\left(\mathbb{R}^{d} ; \mathbb{C}^{n}\right)$ with an error estimate of order $\varepsilon$ :

$$
\left\|\left(\widehat{\mathcal{A}}_{\varepsilon}+I\right)^{-1}-\left(\widehat{\mathcal{A}}^{0}+I\right)^{-1}-\varepsilon K_{1}(\varepsilon)\right\|_{L_{2}\left(\mathbb{R}^{d}\right) \rightarrow H^{1}\left(\mathbb{R}^{d}\right)} \leq C \varepsilon .
$$

We see that here the corrector is given by $K_{1}(\varepsilon)$.

The results for the operator $\mathcal{A}_{\varepsilon}$ with $f \neq \mathbf{1}_{n}$ obtained in [BSu1-5] are similar to (1.6), (1.7), (1.9), but more complicated.

\subsection{Parabolic homogenization problems}

By the method discussed above, it is also possible to study homogenization for a parabolic Cauchy problem. Let $\mathbf{u}_{\varepsilon}(\mathbf{x}, \tau), \mathbf{x} \in \mathbb{R}^{d}, \tau \geq 0$, be the solution of the Cauchy problem

$$
\partial_{\tau} \mathbf{u}_{\varepsilon}=-\widehat{\mathcal{A}}_{\varepsilon} \mathbf{u}_{\varepsilon}, \quad \mathbf{u}_{\varepsilon}(\mathbf{x}, 0)=\phi(\mathbf{x}),
$$

where $\phi \in L_{2}\left(\mathbb{R}^{d} ; \mathbb{C}^{n}\right)$. The solution $\mathbf{u}_{\varepsilon}$ is represented in terms of the operator exponential: $\mathbf{u}_{\varepsilon}=\exp \left(-\widehat{\mathcal{A}}_{\varepsilon} \tau\right) \boldsymbol{\phi}$. Also, we consider more general problem

$$
Q^{\varepsilon}(\mathbf{x}) \partial_{\tau} \mathbf{v}_{\varepsilon}=-\widehat{\mathcal{A}}_{\varepsilon} \mathbf{v}_{\varepsilon}, \quad Q^{\varepsilon}(\mathbf{x}) \mathbf{v}_{\varepsilon}(\mathbf{x}, 0)=\phi(\mathbf{x})
$$


Here $Q(\mathbf{x})$ is a $\Gamma$-periodic positive definite and bounded $(n \times n)$-matrix-valued function. We factorize $Q(\mathbf{x})^{-1}$ as $Q(\mathbf{x})^{-1}=f(\mathbf{x}) f(\mathbf{x})^{*}$ and consider the operator $\mathcal{A}_{\varepsilon}$ (see (1.3)). The solution of problem (1.11) is represented as $\mathbf{v}_{\varepsilon}=f^{\varepsilon} \exp \left(-\mathcal{A}_{\varepsilon} \tau\right)\left(f^{\varepsilon}\right)^{*} \phi$. One can also study the Cauchy problems for the corresponding nonhomogeneous equations.

The problem is reduced to the study of the operator exponential $\exp \left(-\widehat{\mathcal{A}}_{\varepsilon} \tau\right)$ and the bordered operator exponential $f^{\varepsilon} \exp \left(-\mathcal{A}_{\varepsilon} \tau\right)\left(f^{\varepsilon}\right)^{*}$ for small $\varepsilon$. In [Su1,2], the principal term of approximation for these operators for a fixed $\tau>0$ in the operator norm in $L_{2}\left(\mathbb{R}^{d} ; \mathbb{C}^{n}\right)$ was found. Let $\widehat{\mathcal{A}}^{0}$ be the effective operator (1.4). Then

$$
\left\|\exp \left(-\widehat{\mathcal{A}}_{\varepsilon} \tau\right)-\exp \left(-\widehat{\mathcal{A}}^{0} \tau\right)\right\|_{L_{2}\left(\mathbb{R}^{d}\right) \rightarrow L_{2}\left(\mathbb{R}^{d}\right)} \leq C \varepsilon \tau^{-1 / 2}, \quad \tau>0, \quad \varepsilon>0 .
$$

Next, let $\bar{Q}$ be the mean value of $Q(\mathbf{x})$ over the cell $\Omega$, and let $f_{0}:=(\bar{Q})^{-1 / 2}$. We introduce the operator $\mathcal{A}^{0}=f_{0} b(\mathbf{D})^{*} g^{0} b(\mathbf{D}) f_{0}$. Then

$$
\left\|f^{\varepsilon} \exp \left(-\mathcal{A}_{\varepsilon} \tau\right)\left(f^{\varepsilon}\right)^{*}-f_{0} \exp \left(-\mathcal{A}^{0} \tau\right) f_{0}\right\|_{L_{2}\left(\mathbb{R}^{d}\right) \rightarrow L_{2}\left(\mathbb{R}^{d}\right)} \leq C \varepsilon \tau^{-1 / 2}, \quad \tau>0, \quad \varepsilon>0 .
$$

In the paper [V] by E. Vasilevskaya, more accurate approximations than (1.12) and (1.13) were obtained. In particular, it was proved that

$$
\left\|\exp \left(-\widehat{\mathcal{A}}_{\varepsilon} \tau\right)-\exp \left(-\widehat{\mathcal{A}}^{0} \tau\right)-\varepsilon \mathcal{K}(\varepsilon, \tau)\right\|_{L_{2}\left(\mathbb{R}^{d}\right) \rightarrow L_{2}\left(\mathbb{R}^{d}\right)} \leq C \varepsilon^{2} \tau^{-1}, \quad \tau>0, \quad 0<\varepsilon \leq \tau^{1 / 2}
$$

The corrector $\mathcal{K}(\varepsilon, \tau)$ is similar to the elliptic corrector (1.8) and is represented as

$$
\mathcal{K}(\varepsilon, \tau)=\mathcal{K}_{1}(\varepsilon, \tau)+\left(\mathcal{K}_{1}(\varepsilon, \tau)\right)^{*}+\mathcal{K}_{3}(\tau)
$$

The first term $\mathcal{K}_{1}(\varepsilon, \tau)$ corresponds to the traditional corrector used in homogenization theory. It is given by

$$
\mathcal{K}_{1}(\varepsilon, \tau)=\Lambda^{\varepsilon} b(\mathbf{D}) \exp \left(-\widehat{\mathcal{A}}^{0} \tau\right) .
$$

In the study of the Cauchy problem for the nonhomogeneous equation $\partial_{\tau} \mathbf{u}_{\varepsilon}=-\widehat{\mathcal{A}}_{\varepsilon} \mathbf{u}_{\varepsilon}+$ $\mathbf{F}(\mathbf{x}, \tau)$, besides (1.14) estimates for the case $\varepsilon>\tau^{1 / 2}$ are required. In this case, one has to include the smoothing operator $\Pi_{\varepsilon}$ in the corrector (1.15).

\subsection{Main results}

In the present paper, we find approximations for the operator exponential $\exp \left(-\widehat{\mathcal{A}}_{\varepsilon} \tau\right)$ and the bordered operator exponential $f^{\varepsilon} \exp \left(-\mathcal{A}_{\varepsilon} \tau\right)\left(f^{\varepsilon}\right)^{*}$ in the norm of operators acting from $L_{2}\left(\mathbb{R}^{d} ; \mathbb{C}^{n}\right)$ to $H^{1}\left(\mathbb{R}^{d} ; \mathbb{C}^{n}\right)$. In particular, we obtain the estimate

$$
\left\|\exp \left(-\widehat{\mathcal{A}}_{\varepsilon} \tau\right)-\exp \left(-\widehat{\mathcal{A}}^{0} \tau\right)-\varepsilon \mathcal{K}_{1}(\varepsilon, \tau)\right\|_{L_{2}\left(\mathbb{R}^{d}\right) \rightarrow H^{1}\left(\mathbb{R}^{d}\right)} \leq C(\tau) \varepsilon, \quad \tau>0, \quad 0<\varepsilon \leq \tau^{1 / 2},
$$

where $\mathcal{K}_{1}(\varepsilon, \tau)$ is the corrector (1.16), and $C(\tau)=C_{1} \tau^{-1}+C_{2} \tau^{-1 / 2}$.

Comparing (1.14) and (1.17), we see that in parabolic problems, as well as in elliptic ones, the form of the corrector depends on the kind of an operator norm in approximation. In order to approximate the operator $\exp \left(-\widehat{\mathcal{A}}_{\varepsilon} \tau\right)$ in the $\left(L_{2} \rightarrow H^{1}\right)$-norm with an error of order 
$\varepsilon$ it suffices to use the one-term corrector (1.16), while for approximation of this operator in the $\left(L_{2} \rightarrow L_{2}\right)$-norm with an error of order $\varepsilon^{2}$ one has to use the three-term corrector (1.15).

Estimate (1.17) allows one to approximate the solution $\mathbf{u}_{\varepsilon}$ of problem (1.10). Let $\mathbf{u}_{0}$ be the solution of the homogenized problem

$$
\partial_{\tau} \mathbf{u}_{0}=-\widehat{\mathcal{A}}^{0} \mathbf{u}_{0}, \quad \mathbf{u}_{0}(\mathbf{x}, 0)=\phi(\mathbf{x})
$$

Applying (1.17), we have

$$
\left\|\mathbf{u}_{\varepsilon}-\mathbf{u}_{0}-\varepsilon \Lambda^{\varepsilon} b(\mathbf{D}) \mathbf{u}_{0}\right\|_{H^{1}\left(\mathbb{R}^{d}\right)} \leq C(\tau) \varepsilon\|\phi\|_{L_{2}\left(\mathbb{R}^{d}\right)}, \quad \tau>0, \quad 0<\varepsilon \leq \tau^{1 / 2} .
$$

Also, we obtain approximation of the so called "flux" $\mathbf{p}_{\varepsilon}=g^{\varepsilon} b(\mathbf{D}) \mathbf{u}_{\varepsilon}$ in the $L_{2}\left(\mathbb{R}^{d} ; \mathbb{C}^{m}\right)$-norm with an error of order $\varepsilon$.

In the study of the Cauchy problem for the nonhomogeneous equation $\partial_{\tau} \mathbf{u}_{\varepsilon}=-\widehat{\mathcal{A}}_{\varepsilon} \mathbf{u}_{\varepsilon}+$ $\mathbf{F}(\mathbf{x}, \tau)$, besides (1.17) we need to have estimates for the case $\varepsilon>\tau^{1 / 2}$. For this, we have to include the smoothing operator $\Pi_{\varepsilon}$ in the corrector $\mathcal{K}_{1}(\varepsilon, \tau)$. Then it is possible to obtain approximations of the solutions in $H^{1}\left(\mathbb{R}^{d} ; \mathbb{C}^{n}\right)$ and of the fluxes in $L_{2}\left(\mathbb{R}^{d} ; \mathbb{C}^{m}\right)$ also for the nonhomogeneous problem.

Similar results are obtained for more general problem (1.11) and the corresponding nonhomogeneous problem.

\subsection{The method}

Let us explain the way of the proof of estimate (1.17). In essential, (1.17) is reduced to the inequality

$$
\left\|\widehat{\mathcal{A}}_{\varepsilon}^{1 / 2}\left(e^{-\widehat{\mathcal{A}}_{\varepsilon} \tau}-e^{-\widehat{\mathcal{A}}^{0} \tau}-\varepsilon \mathcal{K}_{1}(\varepsilon, \tau)\right)\right\|_{L_{2}\left(\mathbb{R}^{d}\right) \rightarrow L_{2}\left(\mathbb{R}^{d}\right)} \leq C \tau^{-1} \varepsilon, \quad \tau>0, \quad 0<\varepsilon \leq \tau^{1 / 2} .
$$

By the scaling transformation, estimate (1.18) follows from the inequality

$$
\left\|\widehat{\mathcal{A}}^{1 / 2}\left(e^{-\widehat{\mathcal{A}} \tau}-(I+\Lambda b(\mathbf{D})) e^{-\widehat{\mathcal{A}}^{0} \tau}\right)\right\|_{L_{2}\left(\mathbb{R}^{d}\right) \rightarrow L_{2}\left(\mathbb{R}^{d}\right)} \leq C \tau^{-1}, \quad \tau \geq 1
$$

Next, for the proof of (1.19), the operator $\widehat{\mathcal{A}}$ is decomposed in the direct integral of the operators $\widehat{\mathcal{A}}(\mathbf{k})$ acting in $L_{2}\left(\Omega ; \mathbb{C}^{n}\right)$ and depending on the parameter $\mathbf{k} \in \mathbb{R}^{d}$ (the quasimomentum). The operator $\widehat{\mathcal{A}}(\mathbf{k})$ is given by the differential expression $b(\mathbf{D}+\mathbf{k})^{*} g(\mathbf{x}) b(\mathbf{D}+\mathbf{k})$ with periodic boundary conditions. Estimate (1.19) is equivalent to the inequality

$$
\left\|\widehat{\mathcal{A}}(\mathbf{k})^{1 / 2}\left(e^{-\widehat{\mathcal{A}}(\mathbf{k}) \tau}-(I+\Lambda b(\mathbf{D}+\mathbf{k})) e^{-\widehat{\mathcal{A}}^{0}(\mathbf{k}) \tau}\right)\right\|_{L_{2}(\Omega) \rightarrow L_{2}(\Omega)} \leq C \tau^{-1}, \quad \tau \geq 1,
$$

which must be uniform in $\mathbf{k}$.

The main part of investigation is devoted to the study of the operator family $\widehat{\mathcal{A}}(\mathbf{k})$. The operator $\widehat{\mathcal{A}}(\mathbf{k})$ has compact resolvent and depends on $\mathbf{k}$ analytically. This allows one to apply the analytic perturbation theory (with respect to the one-dimensional parameter 
$t=|\mathbf{k}|)$. Herewith, a good deal of considerations can be done in the framework of an abstract operator-theoretic scheme.

In the abstract scheme, we study a family of factorized operators $A(t)=X(t)^{*} X(t)$ acting in some Hilbert space $\mathfrak{H}$. Here $X(t)=X_{0}+t X_{1}$. It is assumed that the point $\lambda_{0}=0$ is an isolated eigenvalue of multiplicity $n$ for the operator $A(0)$. The family $A(t)$ is studied by methods of the analytic perturbation theory. The crucial role is played by the spectral germ $S$ of the operator family $A(t)$. The germ is a selfadjoint operator acting in the $n$-dimensional space $\mathfrak{N}=\operatorname{Ker} A(0)$ and defined in terms of the threshold characteristics of the operator $A(t)$. It is possible to approximate the operator exponential $\exp (-A(t) \tau)$ in terms of some finite rank operator acting in $\mathfrak{N}$. Herewith, the principal term of approximation is given by the operator $\exp \left(-t^{2} S \tau\right)$. Application of this abstract result leads to estimate (1.20).

The method described above shows that the homogenization procedure can be studied as a spectral threshold effect at the bottom of the spectrum of a periodic elliptic operator.

\subsection{A different approach}

Note that the papers [BSu1,2] stimulated interest in homogenization theory to obtaining error estimates in the operator norm. In [Zh], [ZhPas1], estimates of the form (1.6), (1.9) for the acoustics operator and the elasticity operator (which are of the form (1.2)) were obtained by a different (nonspectral) method. This method is related to inclusion of the additional parameter in a problem; this parameter corresponds to the shift of the vectors of the lattice $\Gamma$ by an arbitrary vector of $\Omega$. In [ZhPas2], by a similar method estimates of the form (1.12), (1.17) were obtained for the acoustics operator $\widehat{\mathcal{A}}_{\varepsilon}=-\operatorname{div} g^{\varepsilon}(\mathbf{x}) \nabla$.

\subsection{The plan of the paper}

The paper consists of three chapters. Chapter 1 (Sec. 2-4) contains necessary operatortheoretic material. In Chapter 2 (Sec. 5-10), periodic DO's of the form (1.1), (1.2) are studied. Approximations of the operators $\exp (-\widehat{\mathcal{A}} \tau)$ and $f \exp (-\mathcal{A} \tau) f^{*}$ are obtained (in Sec. 9 and 10, respectively). Chapter 3 (Sec. 11-13) is devoted to parabolic homogenization problems. In Sec. 11, 12, approximations of the operators $\exp \left(-\widehat{\mathcal{A}}_{\varepsilon} \tau\right)$ and $f^{\varepsilon} \exp \left(-\mathcal{A}_{\varepsilon} \tau\right)\left(f^{\varepsilon}\right)^{*}$ are deduced from the results of Chapter 2 by the scaling transformation. In Sec. 13, the results of Sec. 11, 12 are applied to the study of the solutions of parabolic Cauchy problems.

\subsection{Notation}

Let $\mathfrak{H}$ and $\mathfrak{G}$ be separable Hilbert spaces. The symbols $(\cdot, \cdot)_{\mathfrak{H}}$ and $\|\cdot\|_{\mathfrak{H}}$ stand for the inner product and the norm in $\mathfrak{H}$; the symbol $\|\cdot\|_{\mathfrak{H} \rightarrow \mathfrak{G}}$ denotes the norm of a bounded operator acting from $\mathfrak{H}$ to $\mathfrak{G}$. Sometimes we omit indices, if this does not lead to confusion. By $I=I_{\mathfrak{H}}$ we denote the identity operator in $\mathfrak{H}$. If $A: \mathfrak{H} \rightarrow \mathfrak{G}$ is a linear operator, then $\operatorname{Dom} A$ denotes its domain. If $\mathfrak{N}$ is a subspace of $\mathfrak{H}$, then $\mathfrak{N}^{\perp}:=\mathfrak{H} \ominus \mathfrak{N}$. If $P$ is the orthogonal projection of $\mathfrak{H}$ onto $\mathfrak{N}$, then $P^{\perp}$ is the orthogonal projection onto $\mathfrak{N}^{\perp}$. The symbol $\langle\cdot, \cdot\rangle$ stands for 
the standard inner product in $\mathbb{C}^{n},|\cdot|$ is the norm of a vector in $\mathbb{C}^{n} ; \mathbf{1}_{n}$ denotes the unit $(n \times n)$-matrix. If $a$ is an $(n \times n)$-matrix, then $|a|$ denotes the norm of this matrix as a linear operator in $\mathbb{C}^{n}$.

Next, $\mathbf{x}=\left(x_{1}, \ldots, x_{d}\right) \in \mathbb{R}^{d}, i D_{j}=\partial_{j}=\partial / \partial x_{j}, j=1, \ldots, d, \nabla=\operatorname{grad}=\left(\partial_{1}, \ldots, \partial_{d}\right)$, $\mathbf{D}=-i \nabla=\left(D_{1}, \ldots, D_{d}\right)$.

The $L_{p}$-classes of $\mathbb{C}^{n}$-valued functions in a domain $\mathcal{O} \subseteq \mathbb{R}^{d}$ are denoted by $L_{p}\left(\mathcal{O} ; \mathbb{C}^{n}\right)$, $1 \leq p \leq \infty$. The Sobolev classes of order $s$ of $\mathbb{C}^{n}$-valued functions in a domain $\mathcal{O} \subseteq \mathbb{R}^{d}$ are denoted by $H^{s}\left(\mathcal{O} ; \mathbb{C}^{n}\right), s \in \mathbb{R}$. For $n=1$, we write simply $L_{p}(\mathcal{O}), H^{s}(\mathcal{O})$, etc., but sometimes we use such simplified notation also for the spaces of vector-valued or matrixvalued functions.

By $C, c, \mathcal{C}, \mathfrak{C}$ (probably, with indices and marks) we denote various constants in estimates. By $\beta$ (with indices) we denote various absolute constants.

\section{Chapter 1. Abstract operator-theoretic method}

\section{Threshold properties of a factorized operator family}

\subsection{The operator family $A(t)$}

Let $\mathfrak{H}$ and $\mathfrak{H}_{*}$ be complex separable Hilbert spaces. Suppose that $X_{0}: \mathfrak{H} \rightarrow \mathfrak{H}_{*}$ is a densely defined and closed operator, and that $X_{1}: \mathfrak{H} \rightarrow \mathfrak{H}_{*}$ is a bounded operator. We put

$$
X(t):=X_{0}+t X_{1}, \quad \operatorname{Dom} X(t)=\operatorname{Dom} X_{0}, \quad t \in \mathbb{R} .
$$

The family of selfadjoint operators

$$
A(t):=X(t)^{*} X(t), \quad t \in \mathbb{R},
$$

in $\mathfrak{H}$ is our main object. The operator $(2.1)$ is generated by the quadratic form

$$
a(t)[u, u]=\|X(t) u\|_{\mathfrak{H}_{*}}^{2}, \quad u \in \operatorname{Dom} X_{0},
$$

which is closed in $\mathfrak{H}$. We denote $A(0)=X_{0}^{*} X_{0}=: A_{0}$,

$$
\mathfrak{N}:=\operatorname{Ker} A_{0}=\operatorname{Ker} X_{0}, \quad \mathfrak{N}_{*}:=\operatorname{Ker} X_{0}^{*} .
$$

Let $P$ and $P_{*}$ be the orthogonal projections of $\mathfrak{H}$ onto $\mathfrak{N}$ and of $\mathfrak{H}_{*}$ onto $\mathfrak{N}_{*}$, respectively. It is assumed that the point $\lambda_{0}=0$ is an isolated point of the spectrum of $A_{0}$ (an eigenvalue), and that

$$
0<n:=\operatorname{dim} \mathfrak{N}<\infty, \quad n \leq n_{*}:=\operatorname{dim} \mathfrak{N}_{*} \leq \infty .
$$

The distance from the point $\lambda_{0}=0$ to the rest of the spectrum of $A_{0}$ is denoted by $d^{0}$. Let $F(t, s)$ be the spectral projection of the operator $A(t)$ for an interval $[0, s]$. We fix a number $\delta>0$ such that $8 \delta<d^{0}$. It turns out that

$$
F(t, \delta)=F(t, 3 \delta), \quad \operatorname{rank} F(t, \delta)=n \text { for }|t| \leq t_{0}:=\delta^{1 / 2}\left\|X_{1}\right\|^{-1},
$$

see $[\mathrm{BSu} 2, \mathrm{Ch} .1,(1.3)]$. Below we usually write $F(t)$ in place of $F(t, \delta)$. 


\subsection{The operators $Z$ and $R$}

Let $\mathcal{D}:=\operatorname{Dom} X_{0} \cap \mathfrak{N}^{\perp}$, and let $u \in \mathfrak{H}_{*}$. We consider the following equation for an element $\psi \in \mathcal{D}($ cf. [BSu2, Ch. 1, (1.7)]):

$$
X_{0}^{*}\left(X_{0} \psi-u\right)=0
$$

which is understood in the weak sense. It means that $\psi \in \mathcal{D}$ satisfies the identity

$$
\left(X_{0} \psi, X_{0} \zeta\right)_{\mathfrak{H}_{*}}=\left(u, X_{0} \zeta\right)_{\mathfrak{H}_{*}}, \quad \forall \zeta \in \mathcal{D}
$$

Equation (2.3) admits a unique solution $\psi$, and $\left\|X_{0} \psi\right\|_{\mathfrak{H}_{*}} \leq\|u\|_{\mathfrak{H}_{*}}$. Now, let $\omega \in \mathfrak{N}$ and $u=-X_{1} \omega$; the corresponding solution of equation (2.3) is denoted by $\psi(\omega)$. We define a bounded operator $Z: \mathfrak{H} \rightarrow \mathfrak{H}$ by the following relations:

$$
Z \omega=\psi(\omega), \quad \omega \in \mathfrak{N} ; \quad Z x=0, \quad x \in \mathfrak{N}^{\perp} .
$$

Observe that

$$
Z P=Z, \quad P Z=0 .
$$

Now, we introduce an operator $R$ (see [BSu2, Ch. 1, Subsec. 1.2]) as follows:

$$
R: \mathfrak{N} \rightarrow \mathfrak{N}_{*}, \quad R \omega=X_{0} \psi(\omega)+X_{1} \omega \in \mathfrak{N}_{*} .
$$

Another description of $R$ is given by the formula

$$
R=\left.P_{*} X_{1}\right|_{\mathfrak{N}}
$$

\subsection{The spectral germ}

The selfadjoint operator

$$
S=R^{*} R: \mathfrak{N} \rightarrow \mathfrak{N}
$$

is called the spectral germ of the operator family (2.1) at $t=0$ (see [BSu2, Ch. 1, Subsec. 1.3]). From (2.7) and (2.8) it follows that $S=\left.P X_{1}^{*} P_{*} X_{1}\right|_{\mathfrak{N}}$, whence

$$
\|S\| \leq\left\|X_{1}\right\|^{2} \text {. }
$$

The germ $S$ is said to be nondegenerate if $\operatorname{Ker} S=\{0\}$, or equivalently, rank $R=n$.

According to the general analytic perturbation theory (see $[\mathrm{Ka}]$ ), for $|t| \leq t_{0}$ there exist real-analytic functions $\lambda_{l}(t)$ and real-analytic $\mathfrak{H}$-valued functions $\varphi_{l}(t)$ such that

$$
A(t) \varphi_{l}(t)=\lambda_{l}(t) \varphi_{l}(t), \quad l=1, \ldots, n, \quad|t| \leq t_{0},
$$

and $\varphi_{l}(t), l=1, \ldots, n$, form an orthonormal basis in $F(t) \mathfrak{H}$. For sufficiently small $t_{*}\left(\leq t_{0}\right)$ and $|t| \leq t_{*}$, we have the following convergent power series expansions:

$$
\lambda_{l}(t)=\gamma_{l} t^{2}+\mu_{l} t^{3}+\cdots, \quad \gamma_{l} \geq 0, \quad \mu_{l} \in \mathbb{R}, \quad l=1, \ldots, n,
$$




$$
\varphi_{l}(t)=\omega_{l}+t \varphi_{l}^{(1)}+t^{2} \varphi_{l}^{(2)}+\cdots, \quad l=1, \ldots, n .
$$

The elements $\omega_{l}=\varphi_{l}(0), l=1, \ldots, n$, form an orthonormal basis in $\mathfrak{N}$. In [BSu2, Ch. 1, Subsec. 1.6] it was shown that the numbers $\gamma_{l}$ and the elements $\omega_{l}, l=1, \ldots, n$, are eigenvalues and eigenvectors of the operator $S$ :

$$
S \omega_{l}=\gamma_{l} \omega_{l}, \quad l=1, \ldots, n .
$$

\subsection{Threshold approximations}

In $[\mathrm{BSu} 2, \mathrm{Ch} .1$, Theorems 4.1, 4.3] it was shown that

$$
\begin{gathered}
\|F(t)-P\|_{\mathfrak{H} \rightarrow \mathfrak{H}} \leq C_{1}|t|, \quad|t| \leq t_{0}, \quad C_{1}=\beta_{1} \delta^{-1 / 2}\left\|X_{1}\right\|, \\
\left\|A(t) F(t)-t^{2} S P\right\|_{\mathfrak{H} \rightarrow \mathfrak{H}} \leq C_{2}|t|^{3}, \quad|t| \leq t_{0}, \quad C_{2}=\beta_{2} \delta^{-1 / 2}\left\|X_{1}\right\|^{3} .
\end{gathered}
$$

We recall that $\beta$ (with indices) denotes various absolute constants.

Besides (2.12), we need more accurate approximation of the spectral projection $F(t)$ :

$$
F(t)=P+t F_{1}+F_{2}(t)
$$

where $\left\|F_{2}(t)\right\|=O\left(t^{2}\right)$. According to [BSu3, (2.15)], the operator $F_{1}$ is represented as

$$
F_{1}=Z P+P Z^{*}
$$

From (2.15) and (2.6) it follows that

$$
F_{1} P=Z P
$$

Relations (2.13) and (2.9) imply that

$$
\begin{aligned}
\|A(t) F(t)\|_{\mathfrak{H} \rightarrow \mathfrak{H}} & \leq t^{2}\|S\|+C_{2}|t|^{3} \leq C_{3} t^{2}, \quad|t| \leq t_{0}, \\
C_{3} & =\left\|X_{1}\right\|^{2}+C_{2} t_{0}=\left(1+\beta_{2}\right)\left\|X_{1}\right\|^{2} .
\end{aligned}
$$

Here we have used expressions for $t_{0}$ and $C_{2}$ (see (2.2), (2.13)). By (2.17), the eigenvalues $\lambda_{l}(t)$ of the operator $A(t)$ satisfy the estimates $\lambda_{l}(t) \leq C_{3} t^{2}$ for $|t| \leq t_{0}, l=1, \ldots, n$. Consequently,

$$
\left\|A(t)^{1 / 2} F(t)\right\|_{\mathfrak{H} \rightarrow \mathfrak{H}} \leq C_{3}^{1 / 2}|t|, \quad|t| \leq t_{0} .
$$

We also need the estimate for the operator $A(t)^{1 / 2} F_{2}(t)$ proved in [BSu $\left.5,(2.23)\right]$ :

$$
\left\|A(t)^{1 / 2} F_{2}(t)\right\|_{\mathfrak{H} \rightarrow \mathfrak{H}} \leq C_{4} t^{2}, \quad|t| \leq t_{0}, \quad C_{4}=\beta_{3} \delta^{-1 / 2}\left\|X_{1}\right\|^{2} .
$$




\section{Approximation of the operator $\exp (-A(t) \tau)$}

\subsection{The principal term of approximation for $e^{-A(t) \tau}$}

We assume (cf. [BSu2, Ch. 1, (5.1)]) that for some $c_{*}>0$ we have

$$
A(t) \geq c_{*} t^{2} I, \quad|t| \leq t_{0} .
$$

This is equivalent to the fact that the eigenvalues $\lambda_{l}(t)$ of $A(t)$ satisfy the estimates

$$
\lambda_{l}(t) \geq c_{*} t^{2}, \quad|t| \leq t_{0}, \quad l=1, \ldots, n .
$$

Combining this with (2.10), we see that $\gamma_{l} \geq c_{*}, l=1, \ldots, n$. Hence, by $(2.11)$, we have

$$
S \geq c_{*} I_{\mathfrak{N}}
$$

Thus, the germ $S$ is nondegenerate. Obviously, relations (3.1) and (3.3) imply that

$$
\begin{gathered}
\|\exp (-A(t) \tau)\|_{\mathfrak{H} \rightarrow \mathfrak{H}} \leq e^{-c_{*} t^{2} \tau}, \quad \tau>0, \quad|t| \leq t_{0}, \\
\left\|\exp \left(-t^{2} S \tau\right) P\right\|_{\mathfrak{H} \rightarrow \mathfrak{H}} \leq e^{-c_{*} t^{2} \tau}, \quad \tau>0 .
\end{gathered}
$$

In $[\mathrm{Su} 2,(2.11)]$, on the basis of approximations (2.12), (2.13), it was shown that

$$
\left\|e^{-A(t) \tau} F(t)-e^{-t^{2} S \tau} P\right\|_{\mathfrak{H} \rightarrow \mathfrak{H}} \leq\left(2 C_{1}|t|+C_{2}|t|^{3} \tau\right) e^{-c_{*} t^{2} \tau}, \quad \tau>0, \quad|t| \leq t_{0} .
$$

From (3.5) it was deduced (see [Su2, Theorem 2.1]) that for large $\tau$ the principle term of approximation for the operator $\exp (-A(t) \tau)$ is given by the operator $\exp \left(-t^{2} S \tau\right) P$, and

$$
\left\|e^{-A(t) \tau}-e^{-t^{2} S \tau} P\right\|_{\mathfrak{H} \rightarrow \mathfrak{H}} \leq C_{0}(1+\tau)^{-1 / 2}, \quad \tau>0, \quad|t| \leq t_{0} .
$$

The constant $C_{0}$ depends only on $\delta,\left\|X_{1}\right\|$, and $c_{*}$.

More accurate approximation of the operator $\exp (-A(t) \tau)$ in the operator norm in $\mathfrak{H}$ with an error estimate of order $O\left(\tau^{-1}\right)$ was found in [V].

\subsection{Approximation of the operator $e^{-A(t) \tau}$ in the "energetic" norm}

Here we obtain another approximation of the operator $\exp (-A(t) \tau)$ (in the "energetic" norm). Precisely, we study the operator $A(t)^{1 / 2} \exp (-A(t) \tau)$. Our goal is to prove the following theorem.

Theorem 3.1. Suppose that the operator family $A(t)$ satisfies the conditions of Subsection 2.1 and also condition (3.1). Let $P$ be the orthogonal projection of $\mathfrak{H}$ onto the subspace $\mathfrak{N}=\operatorname{Ker} X_{0}$. Let $Z$ be the operator defined by (2.5), and let $S$ be the spectral germ of the family $A(t)$ at $t=0$. Then for $|t| \leq t_{0}$ and $\tau>0$ we have

$$
\left\|A(t)^{1 / 2}\left(e^{-A(t) \tau}-(I+t Z) e^{-t^{2} S \tau} P\right)\right\|_{\mathfrak{H} \rightarrow \mathfrak{H}} \leq C_{5} \tau^{-1}
$$


The number $t_{0}$ is defined by (2.2), and the constant $C_{5}$ is defined below in (3.18); it depends only on $\delta,\left\|X_{1}\right\|$, and $c_{*}$.

Proof. We put

$$
\mathfrak{A}(t, \tau):=A(t)^{1 / 2} \exp (-A(t) \tau)
$$

and represent this operator as

$$
\mathfrak{A}(t, \tau)=\mathfrak{A}(t, \tau) F(t)^{\perp}+\mathfrak{A}(t, \tau) F(t)(F(t)-P)+F(t) \mathfrak{A}(t, \tau) P .
$$

By (2.2) and the obvious inequality $e^{-\alpha} \leq \alpha^{-1}, \alpha>0$, we have:

$$
\left\|\mathfrak{A}(t, \tau) F(t)^{\perp}\right\|_{\mathfrak{H} \rightarrow \mathfrak{H}} \leq \sup _{\lambda \geq 3 \delta} \lambda^{1 / 2} e^{-\lambda \tau} \leq(3 \delta)^{-1 / 2} \tau^{-1}, \quad \tau>0, \quad|t| \leq t_{0} .
$$

Next, by (3.2), for $\tau>0$ and $|t| \leq t_{0}$ we obtain

$$
\|\mathfrak{A}(t, \tau) F(t)\|_{\mathfrak{H} \rightarrow \mathfrak{H}} \leq \sup _{1 \leq l \leq n}\left(\lambda_{l}(t)\right)^{1 / 2} e^{-\lambda_{l}(t) \tau} \leq \tau^{-1} \sup _{1 \leq l \leq n}\left(\lambda_{l}(t)\right)^{-1 / 2} \leq c_{*}^{-1 / 2}|t|^{-1} \tau^{-1} .
$$

Combining this with (2.12), we arrive at the estimate

$$
\|\mathfrak{A}(t, \tau) F(t)(F(t)-P)\|_{\mathfrak{H} \rightarrow \mathfrak{H}} \leq C_{1} c_{*}^{-1 / 2} \tau^{-1}, \quad \tau>0, \quad|t| \leq t_{0} .
$$

The last term in the right-hand side of (3.9) is represented as

$$
F(t) \mathfrak{A}(t, \tau) P=A(t)^{1 / 2} F(t)\left(e^{-A(t) \tau} F(t)-e^{-t^{2} S \tau} P\right) P+A(t)^{1 / 2} F(t) e^{-t^{2} S \tau} P .
$$

From (2.18) and (3.5) it follows that for $\tau>0$ and $|t| \leq t_{0}$ we have

$$
\left\|A(t)^{1 / 2} F(t)\left(e^{-A(t) \tau} F(t)-e^{-t^{2} S \tau} P\right) P\right\|_{\mathfrak{H} \rightarrow \mathfrak{H}} \leq C_{3}^{1 / 2}|t|\left(2 C_{1}|t|+C_{2}|t|^{3} \tau\right) e^{-c_{*} t^{2} \tau} \leq C_{6} \tau^{-1},
$$

where

$$
C_{6}=C_{3}^{1 / 2} \sup _{\alpha>0}\left(2 C_{1} \alpha+C_{2} \alpha^{2}\right) e^{-c_{*} \alpha} .
$$

By (3.8)-(3.13) for $\tau>0$ and $|t| \leq t_{0}$ we obtain:

$$
\begin{aligned}
& \left\|A(t)^{1 / 2}\left(e^{-A(t) \tau}-(I+t Z) e^{-t^{2} S \tau} P\right)\right\|_{\mathfrak{H} \rightarrow \mathfrak{H}} \leq \tau^{-1}\left((3 \delta)^{-1 / 2}+C_{1} c_{*}^{-1 / 2}+C_{6}\right) \\
& \quad+\left\|A(t)^{1 / 2}(F(t) P-P-t Z P) e^{-t^{2} S \tau} P\right\|_{\mathfrak{H} \rightarrow \mathfrak{H} .}
\end{aligned}
$$

From (2.14) and (2.16) it follows that

$$
F(t) P-P-t Z P=\left(P+t F_{1}+F_{2}(t)\right) P-P-t Z P=F_{2}(t) P .
$$

Then, using (2.19) and (3.4), we estimate the last term in the right-hand side of (3.15):

$$
\left\|A(t)^{1 / 2} F_{2}(t) e^{-t^{2} S \tau} P\right\|_{\mathfrak{H} \rightarrow \mathfrak{H}} \leq C_{4} t^{2} e^{-c_{*} t^{2} \tau} \leq C_{4} c_{*}^{-1} \tau^{-1}, \quad \tau>0, \quad|t| \leq t_{0} .
$$

Now, relations (3.15)-(3.17) imply estimate (3.7) with the constant

$$
C_{5}=(3 \delta)^{-1 / 2}+C_{1} c_{*}^{-1 / 2}+C_{6}+C_{4} c_{*}^{-1} \cdot \bullet
$$




\section{Approximation of the bordered exponential}

\subsection{The operator family $A(t)=M^{*} \widehat{A}(t) M$}

Now we consider an operator family of the form $A(t)=M^{*} \widehat{A}(t) M$ (see [BSu2, Ch. 1, Subsec. $1.5,5.3])$. Let $\widehat{\mathfrak{H}}$ be yet another Hilbert space, and let $M: \mathfrak{H} \rightarrow \widehat{\mathfrak{H}}$ be an isomorphism. Let $\widehat{X}(t)=\widehat{X}_{0}+t \widehat{X}_{1}: \widehat{\mathfrak{H}} \rightarrow \mathfrak{H}_{*}$ be a family of the same type as $X(t)$. Suppose that

$$
M \operatorname{Dom} X_{0}=\operatorname{Dom} \widehat{X}_{0}, \quad X_{0}=\widehat{X}_{0} M, \quad X_{1}=\widehat{X}_{1} M .
$$

Then $X(t)=\widehat{X}(t) M$. Consider the family of operators

$$
\widehat{A}(t)=\widehat{X}(t)^{*} \widehat{X}(t): \widehat{\mathfrak{H}} \rightarrow \widehat{\mathfrak{H}}
$$

Obviously,

$$
A(t)=M^{*} \widehat{A}(t) M
$$

In what follows, all the objects corresponding to the family (4.1) are supplied with the upper mark $"$ " . Note that $\widehat{\mathfrak{N}}=M \mathfrak{N}$ and $\widehat{\mathfrak{N}}_{*}=\mathfrak{N}_{*}$. We denote

$$
Q:=\left(M M^{*}\right)^{-1}=\left(M^{*}\right)^{-1} M^{-1}: \widehat{\mathfrak{H}} \rightarrow \widehat{\mathfrak{H}}
$$

The operator $Q$ is positive and continuous together with $Q^{-1}$. Let $Q_{\widehat{\mathfrak{N}}}$ be the block of the operator $Q$ in the subspace $\widehat{\mathfrak{N}}$ :

$$
Q_{\widehat{\mathfrak{N}}}=\left.\widehat{P} Q\right|_{\widehat{\mathfrak{N}}}: \widehat{\mathfrak{N}} \rightarrow \widehat{\mathfrak{N}}
$$

Obviously, $Q_{\widehat{\mathfrak{N}}}$ is an isomorphism in $\widehat{\mathfrak{N}}$. As was shown in [Su2, Proposition 1.2], the orthogonal projection $P$ of the space $\mathfrak{H}$ onto $\mathfrak{N}$ and the orthogonal projection $\widehat{P}$ of the space $\widehat{\mathfrak{H}}$ onto $\widehat{\mathfrak{N}}$ satisfy the following relation:

$$
P=M^{-1}\left(Q_{\widehat{\mathfrak{N}}}\right)^{-1} \widehat{P}\left(M^{*}\right)^{-1}
$$

According to [BSu2, Ch. 1, Subsec. 1.5], for the spectral germs $S$ and $\widehat{S}$ we have

$$
S=\left.P M^{*} \widehat{S} M\right|_{\mathfrak{N}}
$$

Let $\widehat{Z}_{Q}$ be the operator in $\widehat{\mathfrak{H}}$ that takes an element $\widehat{u} \in \widehat{\mathfrak{H}}$ to the solution $\widehat{\psi}_{Q}$ of the equation

$$
\widehat{X}_{0}^{*}\left(\widehat{X}_{0} \widehat{\psi}_{Q}+\widehat{X}_{1} \widehat{\omega}\right)=0, \quad Q \widehat{\psi}_{Q} \perp \widehat{\mathfrak{N}}
$$

where $\widehat{\omega}=\widehat{P} \widehat{u} \in \widehat{\mathfrak{N}}$. Equation (4.7) is understood in the weak sense (cf. (2.4)). Then

$$
\widehat{Z}_{Q}=M Z M^{-1} \widehat{P},
$$

where $Z$ is the operator (2.5) (see [BSu3, Lemma 6.1]). 


\subsection{Approximation of the operator $M e^{-A(t) \tau} M^{*}$}

Under the assumptions of Subsec. 4.1, we find approximation of the operator

$$
M e^{-A(t) \tau} M^{*}=M e^{-M^{*} \widehat{A}(t) M \tau} M^{*}: \widehat{\mathfrak{H}} \rightarrow \widehat{\mathfrak{H}} .
$$

Let $Q_{\widehat{\mathfrak{N}}}$ be the operator defined by (4.4). We put

$$
M_{0}=\left(Q_{\widehat{\mathfrak{N}}}\right)^{-1 / 2}: \widehat{\mathfrak{N}} \rightarrow \widehat{\mathfrak{N}} .
$$

In [Su2, Theorem 2.4], the principle term of approximation for the operator (4.9) was found:

$$
\left\|M e^{-A(t) \tau} M^{*}-M_{0} e^{-t^{2} M_{0} \widehat{S} M_{0} \tau} M_{0} \widehat{P}\right\|_{\widehat{\mathfrak{H}} \rightarrow \widehat{\mathfrak{H}}} \leq C_{0}\|M\|^{2}(1+\tau)^{-1 / 2}, \quad \tau>0, \quad|t| \leq t_{0} .
$$

More accurate approximation for the operator (4.9) in the operator norm in $\mathfrak{H}$ with an error term of order $O\left(\tau^{-1}\right)$ was found in [V].

Now we study the operator $\widehat{A}(t)^{1 / 2} M e^{-A(t) \tau} M^{*}$ and prove the following theorem.

Theorem 4.1. Under the assumptions of Subsec. 4.1, 4.2, for $\tau>0$ and $|t| \leq t_{0}$ we have

$$
\left\|\widehat{A}(t)^{1 / 2}\left(M e^{-A(t) \tau} M^{*}-\left(I+t \widehat{Z}_{Q}\right) M_{0} e^{-t^{2} M_{0} \widehat{S} M_{0} \tau} M_{0} \widehat{P}\right)\right\|_{\widehat{\mathfrak{H}} \rightarrow \widehat{\mathfrak{H}}} \leq C_{5}\|M\| \tau^{-1}
$$

Proof. Estimate (4.12) follows from (3.7) by recalculation. By (4.5) and (4.6), it is easily seen that

$$
M_{0} e^{-t^{2} M_{0} \widehat{S} M_{0} \tau} M_{0} \widehat{P}=M e^{-t^{2} S \tau} P M^{*}
$$

see [Su2, Proposition 2.3]. Combining this with (4.8), we obtain:

$$
\begin{aligned}
& \left\|\widehat{A}(t)^{1 / 2}\left(M e^{-A(t) \tau} M^{*}-\left(I+t \widehat{Z}_{Q}\right) M_{0} e^{-t^{2} M_{0} \widehat{S} M_{0} \tau} M_{0} \widehat{P}\right)\right\|_{\widehat{\mathfrak{H}} \rightarrow \widehat{\mathfrak{H}}} \\
& =\left\|\left(\widehat{X}_{0}+t \widehat{X}_{1}\right) M\left(e^{-A(t) \tau}-(I+t Z) e^{-t^{2} S \tau} P\right) M^{*}\right\|_{\widehat{\mathfrak{H}} \rightarrow \mathfrak{H}_{*}} \\
& =\left\|\left(X_{0}+t X_{1}\right)\left(e^{-A(t) \tau}-(I+t Z) e^{-t^{2} S \tau} P\right) M^{*}\right\|_{\widehat{\mathfrak{H}} \rightarrow \mathfrak{H}_{*}} \\
& \leq\|M\|\left\|A(t)^{1 / 2}\left(e^{-A(t) \tau}-(I+t Z) e^{-t^{2} S \tau} P\right)\right\|_{\mathfrak{H} \rightarrow \mathfrak{H}} .
\end{aligned}
$$

Together with (3.7), this implies (4.12).

\section{Chapter 2. Periodic differential operators}

\section{Matrix periodic DO's. Direct integral expansion}

Here we recall the description of a class of matrix second order differential operators (DO's) admitting a factorization of the form $\mathcal{A}=\mathcal{X}^{*} \mathcal{X}$, where $\mathcal{X}$ is a homogeneous first order DO. This class was introduced and studied in [BSu1,2]. 


\subsection{Factorized second order operators}

Let $b(\mathbf{D}): L_{2}\left(\mathbb{R}^{d} ; \mathbb{C}^{n}\right) \rightarrow L_{2}\left(\mathbb{R}^{d} ; \mathbb{C}^{m}\right)$ be a homogeneous first order DO with constant coefficients. We always assume that $m \geq n$. The symbol $b(\boldsymbol{\xi}), \boldsymbol{\xi} \in \mathbb{R}^{d}$, of the operator $b(\mathbf{D})$ is an $(m \times n)$-matrix-valued linear homogeneous function of $\boldsymbol{\xi}$. It is assumed that

$$
\operatorname{rank} b(\boldsymbol{\xi})=n, \quad 0 \neq \boldsymbol{\xi} \in \mathbb{R}^{d} .
$$

Condition (5.1) is equivalent to the inequalities

$$
\alpha_{0} \mathbf{1}_{n} \leq b(\boldsymbol{\theta})^{*} b(\boldsymbol{\theta}) \leq \alpha_{1} \mathbf{1}_{n}, \quad \boldsymbol{\theta} \in \mathbb{S}^{d-1}, \quad 0<\alpha_{0} \leq \alpha_{1}<\infty
$$

with some constants $\alpha_{0}, \alpha_{1}$. Note that

$$
b(\boldsymbol{\xi})=\sum_{j=1}^{d} b_{j} \xi_{j} ; \quad\left|b_{j}\right| \leq \alpha_{1}^{1 / 2}, \quad j=1, \ldots, d,
$$

where $b_{j}$ are constant $(m \times n)$-matrices.

Suppose that an $(n \times n)$-matrix-valued function $f(\mathbf{x})$ and an $(m \times m)$-matrix-valued function $h(\mathbf{x})$ are bounded, together with their inverses:

$$
f, f^{-1} \in L_{\infty}\left(\mathbb{R}^{d}\right) ; \quad h, h^{-1} \in L_{\infty}\left(\mathbb{R}^{d}\right) .
$$

We consider the DO $\mathcal{X}: L_{2}\left(\mathbb{R}^{d} ; \mathbb{C}^{n}\right) \rightarrow L_{2}\left(\mathbb{R}^{d} ; \mathbb{C}^{m}\right)$ given by

$$
\mathcal{X}=h b(\mathbf{D}) f, \quad \operatorname{Dom} \mathcal{X}=\left\{\mathbf{u} \in L_{2}\left(\mathbb{R}^{d} ; \mathbb{C}^{n}\right): f \mathbf{u} \in H^{1}\left(\mathbb{R}^{d} ; \mathbb{C}^{n}\right)\right\}
$$

The operator $\mathcal{X}$ is closed. The selfadjoint operator $\mathcal{A}(g, f)=\mathcal{A}:=\mathcal{X}^{*} \mathcal{X}$ in $L_{2}\left(\mathbb{R}^{d} ; \mathbb{C}^{n}\right)$ is generated by the closed quadratic form

$$
a[\mathbf{u}, \mathbf{u}]:=\|\mathcal{X} \mathbf{u}\|_{L_{2}\left(\mathbb{R}^{d} ; \mathbb{C}^{m}\right)}^{2}, \quad \mathbf{u} \in \operatorname{Dom} \mathcal{X}
$$

Formally, we have

$$
\mathcal{A}(g, f)=\mathcal{A}=f(\mathbf{x})^{*} b(\mathbf{D})^{*} g(\mathbf{x}) b(\mathbf{D}) f(\mathbf{x}), \quad g(\mathbf{x}):=h(\mathbf{x})^{*} h(\mathbf{x}) .
$$

By using the Fourier transformation and (5.2), (5.4), it is easily seen that

$$
\begin{gathered}
c_{0} \int_{\mathbb{R}^{d}}|\mathbf{D}(f \mathbf{u})|^{2} d \mathbf{x} \leq a[\mathbf{u}, \mathbf{u}] \leq c_{1} \int_{\mathbb{R}^{d}}|\mathbf{D}(f \mathbf{u})|^{2} d \mathbf{x}, \quad \mathbf{u} \in \operatorname{Dom} \mathcal{X} \\
c_{0}=\alpha_{0}\left\|g^{-1}\right\|_{L_{\infty}}^{-1}, \quad c_{1}=\alpha_{1}\|g\|_{L_{\infty}} .
\end{gathered}
$$




\subsection{Lattices $\Gamma$ and $\widetilde{\Gamma}$. The Fourier series}

In what follows, the matrix-valued functions $f$ and $h$ are assumed to be periodic with respect to some lattice $\Gamma \subset \mathbb{R}^{d}$. Let $\mathbf{a}_{1}, \ldots, \mathbf{a}_{d}$ be the basis in $\mathbb{R}^{d}$ that generates this lattice, i. e.,

$$
\Gamma=\left\{\mathbf{a}=n_{1} \mathbf{a}_{1}+\cdots+n_{d} \mathbf{a}_{d}, \quad n_{j} \in \mathbb{Z}\right\}
$$

By $\Omega \subset \mathbb{R}^{d}$ we denote the cell of the lattice $\Gamma$ :

$$
\Omega=\left\{\mathbf{x}=\tau_{1} \mathbf{a}_{1}+\cdots+\tau_{d} \mathbf{a}_{d}, \quad \tau_{j} \in(0,1)\right\}
$$

The basis $\mathbf{b}_{1}, \ldots, \mathbf{b}_{d}$ in $\mathbb{R}^{d}$ dual to the basis $\mathbf{a}_{1}, \ldots, \mathbf{a}_{d}$ is defined by the relations $\left\langle\mathbf{b}_{j}, \mathbf{a}_{l}\right\rangle=$ $2 \pi \delta_{j l}$. Let $\widetilde{\Gamma}$ be the lattice dual to $\Gamma$ :

$$
\widetilde{\Gamma}=\left\{\mathbf{b}=m_{1} \mathbf{b}_{1}+\cdots+m_{d} \mathbf{b}_{d}, \quad m_{j} \in \mathbb{Z}\right\}
$$

By $\widetilde{\Omega}$ we denote the Brillouin zone of the lattice (5.6):

$$
\widetilde{\Omega}=\left\{\mathbf{k} \in \mathbb{R}^{d}: \quad|\mathbf{k}|<|\mathbf{k}-\mathbf{b}|, 0 \neq \mathbf{b} \in \widetilde{\Gamma}\right\} .
$$

Let $r_{0}$ be the radius of the ball inscribed in clos $\widetilde{\Omega}$. Note that $2 r_{0}=\min |\mathbf{b}|, 0 \neq \mathbf{b} \in \widetilde{\Gamma}$.

The following Fourier series expansion for a function $\mathbf{u}(\mathbf{x}), \mathbf{x} \in \Omega$, is associated with the lattice $\Gamma$ :

$$
\mathbf{u}(\mathbf{x})=|\Omega|^{-1 / 2} \sum_{\mathbf{b} \in \widetilde{\Gamma}} \hat{\mathbf{u}}_{\mathbf{b}} \exp (i\langle\mathbf{b}, \mathbf{x}\rangle)
$$

The corresponding discrete Fourier transformation $\left\{\hat{\mathbf{u}}_{\mathbf{b}}\right\} \mapsto \mathbf{u}$ maps $l_{2}\left(\widetilde{\Gamma} ; \mathbb{C}^{n}\right)$ onto $L_{2}\left(\Omega ; \mathbb{C}^{n}\right)$ unitarily:

$$
\int_{\Omega}|\mathbf{u}(\mathbf{x})|^{2} d \mathbf{x}=\sum_{\mathbf{b} \in \widetilde{\Gamma}}\left|\hat{\mathbf{u}}_{\mathbf{b}}\right|^{2}
$$

By $\widetilde{H}^{s}\left(\Omega ; \mathbb{C}^{n}\right)$ we denote the subspace of all functions in $H^{s}\left(\Omega ; \mathbb{C}^{n}\right)$ whose $\Gamma$-periodic extensions to $\mathbb{R}^{d}$ belong to $H_{\text {loc }}^{s}\left(\mathbb{R}^{d} ; \mathbb{C}^{n}\right)$. We have

$$
\int_{\Omega}|(\mathbf{D}+\mathbf{k}) \mathbf{u}|^{2} d \mathbf{x}=\sum_{\mathbf{b} \in \widetilde{\Gamma}}|\mathbf{b}+\mathbf{k}|^{2}\left|\hat{\mathbf{u}}_{\mathbf{b}}\right|^{2}, \quad \mathbf{u} \in \widetilde{H}^{1}\left(\Omega ; \mathbb{C}^{n}\right), \quad \mathbf{k} \in \mathbb{R}^{d}
$$

and convergence of the series in the right-hand side of (5.9) is equivalent to the inclusion $\mathbf{u} \in \widetilde{H}^{1}\left(\Omega ; \mathbb{C}^{n}\right)$. From (5.7)-(5.9) it follows that

$$
\int_{\Omega}|(\mathbf{D}+\mathbf{k}) \mathbf{u}|^{2} d \mathbf{x} \geq \sum_{\mathbf{b} \in \widetilde{\Gamma}}|\mathbf{k}|^{2}\left|\hat{\mathbf{u}}_{\mathbf{b}}\right|^{2}=|\mathbf{k}|^{2} \int_{\Omega}|\mathbf{u}|^{2} d \mathbf{x}, \quad \mathbf{u} \in \widetilde{H}^{1}\left(\Omega ; \mathbb{C}^{n}\right), \quad \mathbf{k} \in \widetilde{\Omega}
$$




\subsection{The Gelfand transformation}

Initially, the Gelfand transformation $\mathcal{V}$ is defined on the functions $\mathbf{u} \in \mathcal{S}\left(\mathbb{R}^{d} ; \mathbb{C}^{n}\right)$ of the Schwartz class by the formula

$$
\widetilde{\mathbf{u}}(\mathbf{k}, \mathbf{x})=(\mathcal{V} \mathbf{u})(\mathbf{k}, \mathbf{x})=|\widetilde{\Omega}|^{-1 / 2} \sum_{\mathbf{a} \in \Gamma} \exp (-i\langle\mathbf{k}, \mathbf{x}+\mathbf{a}\rangle) \mathbf{u}(\mathbf{x}+\mathbf{a}), \quad \mathbf{x} \in \Omega, \quad \mathbf{k} \in \widetilde{\Omega} .
$$

Since

$$
\int_{\widetilde{\Omega}} \int_{\Omega}|\widetilde{\mathbf{u}}(\mathbf{k}, \mathbf{x})|^{2} d \mathbf{x} d \mathbf{k}=\int_{\mathbb{R}^{d}}|\mathbf{u}(\mathbf{x})|^{2} d \mathbf{x}, \quad \widetilde{\mathbf{u}}=\mathcal{V} \mathbf{u}
$$

$\mathcal{V}$ extends by continuity to a unitary mapping

$$
\mathcal{V}: L_{2}\left(\mathbb{R}^{d} ; \mathbb{C}^{n}\right) \rightarrow \int_{\widetilde{\Omega}} \oplus L_{2}\left(\Omega ; \mathbb{C}^{n}\right) d \mathbf{k}=: \mathcal{K}
$$

The relation $\mathbf{u} \in H^{1}\left(\mathbb{R}^{d} ; \mathbb{C}^{n}\right)$ is equivalent to the fact that $\widetilde{\mathbf{u}}(\mathbf{k}, \cdot) \in \widetilde{H}^{1}\left(\Omega ; \mathbb{C}^{n}\right)$ for almost every $\mathbf{k} \in \widetilde{\Omega}$ and

$$
\int_{\widetilde{\Omega}} \int_{\Omega}\left(|(\mathbf{D}+\mathbf{k}) \widetilde{\mathbf{u}}(\mathbf{k}, \mathbf{x})|^{2}+|\widetilde{\mathbf{u}}(\mathbf{k}, \mathbf{x})|^{2}\right) d \mathbf{x} d \mathbf{k}<\infty
$$

\subsection{The forms $a(\mathrm{k})$ and the operators $\mathcal{A}(\mathrm{k})$}

We put $\mathfrak{H}=L_{2}\left(\Omega ; \mathbb{C}^{n}\right), \mathfrak{H}_{*}=L_{2}\left(\Omega ; \mathbb{C}^{m}\right)$, and consider the operator $\mathcal{X}(\mathbf{k}): \mathfrak{H} \rightarrow \mathfrak{H}_{*}, \mathbf{k} \in \mathbb{R}^{d}$, defined by the relations

$$
\mathcal{X}(\mathbf{k})=h b(\mathbf{D}+\mathbf{k}) f, \quad \operatorname{Dom} \mathcal{X}(\mathbf{k})=\left\{\mathbf{u} \in \mathfrak{H}: \quad f \mathbf{u} \in \widetilde{H}^{1}\left(\Omega ; \mathbb{C}^{n}\right)\right\}=: \mathfrak{d} .
$$

The operator $\mathcal{X}(\mathbf{k})$ is closed. The selfadjoint operator

$$
\mathcal{A}(\mathbf{k}):=\mathcal{X}(\mathbf{k})^{*} \mathcal{X}(\mathbf{k}): \mathfrak{H} \rightarrow \mathfrak{H}
$$

is generated by the closed quadratic form

$$
a(\mathbf{k})[\mathbf{u}, \mathbf{u}]:=\|\mathcal{X}(\mathbf{k}) \mathbf{u}\|_{\mathfrak{H}_{*}}^{2}, \quad \mathbf{u} \in \mathfrak{d} .
$$

Using the Fourier series and conditions (5.2), (5.4), it is easy to check that

$$
c_{0} \int_{\Omega}|(\mathbf{D}+\mathbf{k}) \mathbf{v}|^{2} d \mathbf{x} \leq a(\mathbf{k})[\mathbf{u}, \mathbf{u}] \leq c_{1} \int_{\Omega}|(\mathbf{D}+\mathbf{k}) \mathbf{v}|^{2} d \mathbf{x}, \quad \mathbf{v}=f \mathbf{u} \in \widetilde{H}^{1}\left(\Omega ; \mathbb{C}^{n}\right),
$$

were $c_{0}$ and $c_{1}$ are defined in (5.5) (cf. [BSu2, Ch. 2, (2.6)]). From (5.13) and the compactness of the embedding of $\widetilde{H}^{1}\left(\Omega ; \mathbb{C}^{n}\right)$ in $L_{2}\left(\Omega ; \mathbb{C}^{n}\right)$ it follows that the spectrum of $\mathcal{A}(\mathbf{k})$ is discrete. Observe also that the resolvent of the operator $\mathcal{A}(\mathbf{k})$ is compact and depends on $\mathbf{k} \in \mathbb{R}^{d}$ continuously (in the operator norm). Let

$$
\mathfrak{N}:=\operatorname{Ker} \mathcal{A}(0)=\operatorname{Ker} \mathcal{X}(0) .
$$


Relations (5.13) with $\mathbf{k}=0$ show that

$$
\mathfrak{N}=\left\{\mathbf{u} \in L_{2}\left(\Omega ; \mathbb{C}^{n}\right): \quad f \mathbf{u}=\mathbf{c} \in \mathbb{C}^{n}\right\}, \quad \operatorname{dim} \mathfrak{N}=n .
$$

From the lower estimate (5.13) and from (5.10) it follows that

$$
\mathcal{A}(\mathbf{k}) \geq c_{*}|\mathbf{k}|^{2} I, \quad \mathbf{k} \in \widetilde{\Omega},
$$

where

$$
c_{*}=\alpha_{0}\left\|g^{-1}\right\|_{L_{\infty}}^{-1}\left\|f^{-1}\right\|_{L_{\infty}}^{-2}
$$

\subsection{The direct integral for the operator $\mathcal{A}$}

The operators $\mathcal{A}(\mathbf{k})$ allow us to partially diagonalize the operator $\mathcal{A}$ in the direct integral $\mathcal{K}$ (see (5.11)). Let $\widetilde{\mathbf{u}}=\mathcal{V} \mathbf{u}, \mathbf{u} \in \operatorname{Dom} a$. Then

$$
\begin{gathered}
\widetilde{\mathbf{u}}(\mathbf{k}, \cdot) \in \mathfrak{d} \text { for a. e. } \mathbf{k} \in \widetilde{\Omega} \\
a[\mathbf{u}, \mathbf{u}]=\int_{\widetilde{\Omega}} a(\mathbf{k})[\widetilde{\mathbf{u}}(\mathbf{k}, \cdot), \widetilde{\mathbf{u}}(\mathbf{k}, \cdot)] d \mathbf{k} .
\end{gathered}
$$

Conversely, if $\widetilde{\mathbf{u}} \in \mathcal{K}$ satisfies (5.18) and the integral in (5.19) is finite, then $\mathbf{u} \in$ Dom $a$ and (5.19) is valid. The above arguments show that, under the Gelfand transformation, the operator $\mathcal{A}$ expands in the direct integral of the operators $\mathcal{A}(\mathbf{k})$ :

$$
\mathcal{V} \mathcal{A} \mathcal{V}^{-1}=\int_{\widetilde{\Omega}} \oplus \mathcal{A}(\mathbf{k}) d \mathbf{k}
$$

\section{The effective characteristics for the operators $\mathcal{A}(\mathbf{k})$}

\subsection{Incorporation of the operators $\mathcal{A}(\mathrm{k})$ in the general scheme}

For $\mathbf{k} \in \mathbb{R}^{d}$ we put $\mathbf{k}=t \boldsymbol{\theta}, t=|\mathbf{k}|, \boldsymbol{\theta} \in \mathbb{S}^{d-1}$, and view $t$ as the perturbation parameter. At the same time, we must make our constructions and estimates uniform in $\boldsymbol{\theta}$.

We apply the scheme of Sec. 2, putting $\mathfrak{H}=L_{2}\left(\Omega ; \mathbb{C}^{n}\right), \mathfrak{H}_{*}=L_{2}\left(\Omega ; \mathbb{C}^{m}\right)$. The role of $X(t)$ is played by the operator $X(t ; \boldsymbol{\theta})=\mathcal{X}(t \boldsymbol{\theta})=X_{0}+t X_{1}(\boldsymbol{\theta})$, where $X_{0}=\mathcal{X}(0)=$ $h b(\mathbf{D}) f$, Dom $X_{0}=\mathfrak{d}$, and $X_{1}(\boldsymbol{\theta})=h b(\boldsymbol{\theta}) f$. The role of $A(t)$ is played by the operator $A(t ; \boldsymbol{\theta})=\mathcal{A}(t \boldsymbol{\theta})$. According to (5.12), $A(t ; \boldsymbol{\theta})=X(t ; \boldsymbol{\theta})^{*} X(t ; \boldsymbol{\theta})$. By (5.14) and (5.15), we have $\mathfrak{N}=\operatorname{Ker} X_{0}=\operatorname{Ker} \mathcal{X}(0), \operatorname{dim} \mathfrak{N}=n$. The condition $m \geq n$ ensures that $n \leq n_{*}$. Moreover, the following alternative takes place: either $n_{*}=\infty$ (if $m>n$ ), or $n_{*}=n$ (if $m=n)$; see [BSu2, Ch. 2, §3]. As was shown in [BSu2, Ch. 2, §3], the distance $d^{0}$ from the point $\lambda_{0}=0$ to the rest of the spectrum of $\mathcal{A}(0)$ satisfies the estimate

$$
d^{0} \geq 4 c_{*} r_{0}^{2} .
$$


Thus, all the assumptions of Sec. 2 are satisfied. In Sec. 2, it was required to choose a positive number $\delta$ such that $8 \delta<d^{0}$. Using (6.1), we fix $\delta$ as follows:

$$
\delta=c_{*} r_{0}^{2} / 4=\left(r_{0} / 2\right)^{2} \alpha_{0}\left\|f^{-1}\right\|_{L_{\infty}}^{-2}\left\|g^{-1}\right\|_{L_{\infty}}^{-1} .
$$

The estimate $\left\|X_{1}(\boldsymbol{\theta})\right\| \leq \alpha_{1}^{1 / 2}\|f\|_{L_{\infty}}\|h\|_{L_{\infty}}$ allows us to choose $t_{0}$ equal not to $\delta^{1 / 2}\left\|X_{1}(\boldsymbol{\theta})\right\|^{-1}$ (see $(2.2)$ ) but to a smaller number independent of $\boldsymbol{\theta}$. Namely, we put

$$
t_{0}=\delta^{1 / 2} \alpha_{1}^{-1 / 2}\|f\|_{L_{\infty}}^{-1}\|h\|_{L_{\infty}}^{-1}=\left(r_{0} / 2\right) \alpha_{0}^{1 / 2} \alpha_{1}^{-1 / 2}\|f\|_{L_{\infty}}^{-1}\left\|f^{-1}\right\|_{L_{\infty}}^{-1}\|g\|_{L_{\infty}}^{-1 / 2}\left\|g^{-1}\right\|_{L_{\infty}}^{-1 / 2} .
$$

From (6.3) it follows that $t_{0} \leq r_{0} / 2$.

Finally, condition (3.1) for $A(t ; \boldsymbol{\theta})$ is satisfied due to (5.16).

\subsection{The operator $\widehat{\mathcal{A}}(g):=\mathcal{A}\left(g, \mathbf{1}_{n}\right)$ and the matrix $g^{0}$}

In the case where $f=\mathbf{1}_{n}$, we agree to mark all the objects by the upper hat " $\leadsto$ ". For the operator $\widehat{\mathcal{A}}=\widehat{\mathcal{A}}(g)=b(\mathbf{D})^{*} g(\mathbf{x}) b(\mathbf{D})$, the family

$$
\widehat{\mathcal{A}}(\mathbf{k})=b(\mathbf{D}+\mathbf{k})^{*} g(\mathbf{x}) b(\mathbf{D}+\mathbf{k})
$$

is denoted by $\widehat{A}(t ; \boldsymbol{\theta})$. If $f=\mathbf{1}_{n}$, the kernel (5.15) takes the form

$$
\widehat{\mathfrak{N}}=\left\{\mathbf{u} \in \mathfrak{H}: \mathbf{u}=\mathbf{c} \in \mathbb{C}^{n}\right\}
$$

Let $\widehat{P}$ be the orthogonal projection of $\mathfrak{H}$ onto the subspace (6.5). Then

$$
\widehat{P} \mathbf{u}=|\Omega|^{-1} \int_{\Omega} \mathbf{u}(\mathbf{x}) d \mathbf{x}, \quad \mathbf{u} \in \mathfrak{H} .
$$

In other words, $\widehat{P}$ is the operator of averaging over the cell.

Inequality (5.16) with $f=\mathbf{1}_{n}$ shows that

$$
\widehat{\mathcal{A}}(\mathbf{k})=\widehat{A}(t ; \boldsymbol{\theta}) \geq \widehat{c}_{*} t^{2} I, \quad \mathbf{k}=t \boldsymbol{\theta} \in \widetilde{\Omega},
$$

where

$$
\widehat{c}_{*}=\alpha_{0}\left\|g^{-1}\right\|_{L_{\infty}}^{-1} .
$$

The spectral germ $\widehat{S}(\boldsymbol{\theta})$ of the family $\widehat{A}(t ; \boldsymbol{\theta})$ acts in $\widehat{\mathfrak{N}}$ and depends on $\boldsymbol{\theta}$. From (6.7) it follows that the germ is nondegenerate uniformly in $\boldsymbol{\theta}$ (see Subsec. 3.1):

$$
\widehat{S}(\boldsymbol{\theta}) \geq \widehat{c}_{*} I_{\widehat{\mathfrak{N}}}, \quad \boldsymbol{\theta} \in \mathbb{S}^{d-1} .
$$

According to [BSu2, Ch. 3, $\S 1$, the spectral germ $\widehat{S}(\boldsymbol{\theta})$ is represented as

$$
\widehat{S}(\boldsymbol{\theta})=b(\boldsymbol{\theta})^{*} g^{0} b(\boldsymbol{\theta}), \quad \boldsymbol{\theta} \in \mathbb{S}^{d-1},
$$


where $g^{0}$ is a constant positive $(m \times m)$-matrix called the effective matrix.

To define $g^{0}$, we introduce a $\Gamma$-periodic $(n \times m)$-matrix-valued function $\Lambda \in \widetilde{H}^{1}(\Omega)$ which is the solution of the problem

$$
b(\mathbf{D})^{*} g(\mathbf{x})\left(b(\mathbf{D}) \Lambda(\mathbf{x})+\mathbf{1}_{m}\right)=0, \quad \int_{\Omega} \Lambda(\mathbf{x}) d \mathbf{x}=0 .
$$

The equation in (6.11) is understood in the weak sense. Next, we define the $(m \times m)$-matrixvalued function

$$
\widetilde{g}(\mathbf{x})=g(\mathbf{x})\left(b(\mathbf{D}) \Lambda(\mathbf{x})+\mathbf{1}_{m}\right) .
$$

The effective matrix $g^{0}$ is given by the relation

$$
g^{0}=|\Omega|^{-1} \int_{\Omega} \widetilde{g}(\mathbf{x}) d \mathbf{x} .
$$

We also introduce the following operator with constant coefficients

$$
\widehat{\mathcal{A}}^{0}=\widehat{\mathcal{A}}\left(g^{0}\right)=b(\mathbf{D})^{*} g^{0} b(\mathbf{D}),
$$

and the corresponding operator family $\widehat{\mathcal{A}}^{0}(\mathbf{k})$ acting in $L_{2}\left(\Omega ; \mathbb{C}^{n}\right)$ and given by the expression

$$
\widehat{\mathcal{A}}^{0}(\mathbf{k})=b(\mathbf{D}+\mathbf{k})^{*} g^{0} b(\mathbf{D}+\mathbf{k})
$$

with periodic boundary conditions. The operator (6.14) is called the effective operator for $\widehat{\mathcal{A}}(g)$. By (6.10) and (6.9), the symbol of the operator $\widehat{\mathcal{A}}^{0}$ satisfies the estimate

$$
b(\mathbf{k})^{*} g^{0} b(\mathbf{k}) \geq \widehat{c}_{*}|\mathbf{k}|^{2} \mathbf{1}_{n}, \quad \mathbf{k} \in \mathbb{R}^{d} .
$$

Then, estimating the quadratic form of the operator (6.15) by using the Fourier series and (5.10), we obtain

$$
\widehat{\mathcal{A}}^{0}(\mathbf{k}) \geq \widehat{c}_{*}|\mathbf{k}|^{2} I, \quad \mathbf{k} \in \widetilde{\Omega} .
$$

\subsection{Properties of the effective matrix $g^{0}$}

Concerning the following properties of $g^{0}$, see [BSu2, Ch. 3, $\left.§ 1\right]$.

Proposition 6.1. The effective matrix satisfies the estimates

$$
\underline{g} \leq g^{0} \leq \bar{g}
$$

where

$$
\bar{g}:=|\Omega|^{-1} \int_{\Omega} g(\mathbf{x}) d \mathbf{x}, \quad \underline{g}^{-1}:=|\Omega|^{-1} \int_{\Omega} g(\mathbf{x})^{-1} d \mathbf{x} .
$$

If $m=n$, then the effective matrix $g^{0}$ coincides with $\underline{g}: g^{0}=\underline{g}$.

In homogenization theory, estimates (6.18) are known as the Voight-Reuss bracketing. From (6.18) it follows that $\left|g^{0}\right| \leq\|g\|_{L_{\infty}},\left|\left(g^{0}\right)^{-1}\right| \leq\left\|g^{-1}\right\|_{L_{\infty}}$. 
Now, we distinguish the cases where one of the inequalities in (6.18) becomes equality. The following statements were obtained in [BSu2, Ch. 3, Propositions 1.6 and 1.7].

Proposition 6.2. The identity $g^{0}=\bar{g}$ is equivalent to the relations

$$
b(\mathbf{D})^{*} \mathbf{g}_{k}(\mathbf{x})=0, \quad k=1, \ldots, m,
$$

where $\mathbf{g}_{k}(\mathbf{x}), k=1, \ldots, m$, are the columns of the matrix $g(\mathbf{x})$.

Note that condition (6.19) implies that $\Lambda(\mathbf{x})=0$.

Proposition 6.3. The identity $g^{0}=g$ is equivalent to the representations

$$
\mathbf{l}_{k}(\mathbf{x})=\mathbf{l}_{k}^{0}+b(\mathbf{D}) \mathbf{w}_{k}, \quad \mathbf{l}_{k}^{0} \in \mathbb{C}^{m}, \quad \mathbf{w}_{k} \in \widetilde{H}^{1}\left(\Omega ; \mathbb{C}^{n}\right), \quad k=1, \ldots, m,
$$

where $\mathbf{l}_{k}(\mathbf{x}), k=1, \ldots, m$, are the columns of the matrix $g(\mathbf{x})^{-1}$.

Note that under conditions (6.20) the matrix $\widetilde{g}(\mathbf{x})$ defined by $(6.12)$ coincides with $\underline{g}$, i. e., $\widetilde{g}(\mathbf{x})=g^{0}=\underline{g}($ see $[\mathrm{BSu} 4$, Remark 3.5]).

\section{Approximation of the operator $\exp (-\widehat{\mathcal{A}}(\mathbf{k}) \tau)$}

\subsection{Main theorem}

In the present section our main goal is to prove the following theorem.

Theorem 7.1. Let $\widehat{\mathcal{A}}(\mathbf{k})$ be the operator family $(6.4)$, and let $\widehat{\mathcal{A}}^{0}(\mathbf{k})$ be the effective operator family (6.15). Suppose that the matrix-valued $\Gamma$-periodic function $\Lambda(\mathbf{x})$ is the solution of problem (6.11). Let $\widehat{P}$ be the orthogonal projection (6.6). Then for $\mathbf{k} \in \widetilde{\Omega}$ and $\tau>0$ we have

$$
\begin{gathered}
\left\|\widehat{\mathcal{A}}(\mathbf{k})^{1 / 2}\left(e^{-\widehat{\mathcal{A}}(\mathbf{k}) \tau}-(I+\Lambda b(\mathbf{D}+\mathbf{k}) \widehat{P}) e^{-\widehat{\mathcal{A}}^{0}(\mathbf{k}) \tau}\right)\right\|_{L_{2}(\Omega) \rightarrow L_{2}(\Omega)} \leq \Phi_{1}(\tau) \\
\Phi_{1}(\tau)=\left\{\begin{array}{c}
\mathcal{C}_{1} \tau^{-1}, \quad \tau \geq 1 \\
\mathcal{C}_{2} \tau^{-1 / 2}, \quad 0<\tau<1 .
\end{array}\right.
\end{gathered}
$$

The constants $\mathcal{C}_{1}$ and $\mathcal{C}_{2}$ are defined below in (7.24) and (7.29) respectively; they depend only on $m, \alpha_{0}, \alpha_{1},\|g\|_{L_{\infty}},\left\|g^{-1}\right\|_{L_{\infty}}$, and the parameters of the lattice $\Gamma$.

Note that the left-hand side of (7.1) can be estimated by $\mathcal{C}_{1} \tau^{-1}$ as well as by $\mathcal{C}_{2} \tau^{-1 / 2}$ for all $\tau>0$, but for $\tau \geq 1$ the first estimate is preferable, while for $0<\tau<1$ the second one is preferable.

Estimate (7.1) is most informative for $\tau \geq 1$ and $|\mathbf{k}| \leq \widehat{t_{0}}$, where $\widehat{t}_{0}$ is defined according to (6.3) with $f=\mathbf{1}_{n}$ (see (7.6) below). For $\mathbf{k} \in \widetilde{\Omega},|\mathbf{k}|>\widehat{t}_{0}$, and also for $0<\tau<1$, estimate (7.1) is rougher, since each term in (7.1) is estimated separately; however, these cases also require attention. 


\subsection{Estimates in the case $|\mathbf{k}| \leq \widehat{t}_{0}$}

We apply Theorem 3.1 to the operator $\widehat{A}(t ; \boldsymbol{\theta})=\widehat{\mathcal{A}}(\mathbf{k})$. By $(6.6),(6.10)$, and (6.15), we have

$$
t^{2} \widehat{S}(\boldsymbol{\theta}) \widehat{P}=b(\mathbf{k})^{*} g^{0} b(\mathbf{k}) \widehat{P}=b(\mathbf{D}+\mathbf{k})^{*} g^{0} b(\mathbf{D}+\mathbf{k}) \widehat{P}=\widehat{\mathcal{A}}^{0}(\mathbf{k}) \widehat{P}, \quad \mathbf{k} \in \widetilde{\Omega},
$$

whence

$$
\exp \left(-t^{2} \widehat{S}(\boldsymbol{\theta}) \tau\right) \widehat{P}=\exp \left(-\widehat{\mathcal{A}}^{0}(\mathbf{k}) \tau\right) \widehat{P}, \quad \mathbf{k} \in \widetilde{\Omega} .
$$

According to $[\mathrm{BSu} 4,(4.2)]$, the role of $Z$ is played by the operator $\widehat{Z}(\boldsymbol{\theta})=\Lambda b(\boldsymbol{\theta}) \widehat{P}$, where $\Lambda$ denotes the operator of multiplication by the matrix-valued function $\Lambda(\mathbf{x})$. Then

$$
t \widehat{Z}(\boldsymbol{\theta}) \widehat{P}=\Lambda b(\mathbf{k}) \widehat{P}=\Lambda b(\mathbf{D}+\mathbf{k}) \widehat{P} .
$$

Estimate (3.7) is applicable. We only need to specify the constants. The constant $\widehat{c}_{*}$ is defined by (6.8); $\widehat{\delta}$ and $\widehat{t}_{0}$ are defined as in (6.2) and (6.3) with $f=\mathbf{1}_{n}$. Namely,

$$
\begin{gathered}
\widehat{\delta}=\left(r_{0} / 2\right)^{2} \alpha_{0}\left\|g^{-1}\right\|_{L_{\infty}}^{-1}, \\
\widehat{t}_{0}=\widehat{\delta}^{1 / 2} \alpha_{1}^{-1 / 2}\|g\|_{L_{\infty}}^{-1 / 2}=\left(r_{0} / 2\right) \alpha_{0}^{1 / 2} \alpha_{1}^{-1 / 2}\|g\|_{L_{\infty}}^{-1 / 2}\left\|g^{-1}\right\|_{L_{\infty}}^{-1 / 2} .
\end{gathered}
$$

Using that $\left\|\widehat{X}_{1}(\boldsymbol{\theta})\right\| \leq \alpha_{1}^{1 / 2}\|g\|_{L_{\infty}}^{1 / 2}$, instead of the precise values $\widehat{C}_{1}(\boldsymbol{\theta})=\beta_{1} \widehat{\delta}^{-1 / 2}\left\|\widehat{X}_{1}(\boldsymbol{\theta})\right\|$ and $\widehat{C}_{2}(\boldsymbol{\theta})=\beta_{2} \widehat{\delta}^{-1 / 2}\left\|\widehat{X}_{1}(\boldsymbol{\theta})\right\|^{3}$ (see Subsec. 2.4) we can take

$$
\begin{aligned}
& \widehat{C}_{1}=\beta_{1} \widehat{\delta}^{-1 / 2} \alpha_{1}^{1 / 2}\|g\|_{L_{\infty}}^{1 / 2}=2 \beta_{1} r_{0}^{-1} \alpha_{0}^{-1 / 2} \alpha_{1}^{1 / 2}\|g\|_{L_{\infty}}^{1 / 2}\left\|g^{-1}\right\|_{L_{\infty}}^{1 / 2}, \\
& \widehat{C}_{2}=\beta_{2} \widehat{\delta}^{-1 / 2} \alpha_{1}^{3 / 2}\|g\|_{L_{\infty}}^{3 / 2}=2 \beta_{2} r_{0}^{-1} \alpha_{0}^{-1 / 2} \alpha_{1}^{3 / 2}\|g\|_{L_{\infty}}^{3 / 2}\left\|g^{-1}\right\|_{L_{\infty}}^{1 / 2} .
\end{aligned}
$$

Similarly, instead of the precise values $\widehat{C}_{3}(\boldsymbol{\theta})=\left(1+\beta_{2}\right)\left\|\widehat{X}_{1}(\boldsymbol{\theta})\right\|^{2}, \widehat{C}_{4}(\boldsymbol{\theta})=\beta_{3} \widehat{\delta}^{-1 / 2}\left\|\widehat{X}_{1}(\boldsymbol{\theta})\right\|^{2}$ (see $(2.17),(2.19))$, we take

$$
\begin{gathered}
\widehat{C}_{3}=\left(1+\beta_{2}\right) \alpha_{1}\|g\|_{L_{\infty}}, \\
\widehat{C}_{4}=\beta_{3} \widehat{\delta}^{-1 / 2} \alpha_{1}\|g\|_{L_{\infty}}=2 \beta_{3} r_{0}^{-1} \alpha_{0}^{-1 / 2} \alpha_{1}\|g\|_{L_{\infty}}\left\|g^{-1}\right\|_{L_{\infty}}^{1 / 2} .
\end{gathered}
$$

Next, we put (see (3.14))

$$
\widehat{C}_{6}=\widehat{C}_{3}^{1 / 2} \sup _{\alpha>0}\left(2 \widehat{C}_{1} \alpha+\widehat{C}_{2} \alpha^{2}\right) \exp \left(-\widehat{c}_{*} \alpha\right) .
$$

Applying Theorem 3.1 to the operator family $\widehat{A}(t ; \boldsymbol{\theta})=\widehat{\mathcal{A}}(\mathbf{k})$ and taking $(7.3)$ and $(7.4)$ into account, we obtain the estimate

$$
\left\|\widehat{\mathcal{A}}(\mathbf{k})^{1 / 2}\left(e^{-\widehat{\mathcal{A}}(\mathbf{k}) \tau}-(I+\Lambda b(\mathbf{D}+\mathbf{k})) e^{-\widehat{\mathcal{A}}^{0}(\mathbf{k}) \tau} \widehat{P}\right)\right\|_{L_{2}(\Omega) \rightarrow L_{2}(\Omega)} \leq \widehat{C}_{5} \tau^{-1}, \quad \tau>0, \quad|\mathbf{k}| \leq \widehat{t}_{0}
$$

where $\widehat{t}_{0}$ is defined by (7.6), and the constant $\widehat{C}_{5}$ is given by

$$
\widehat{C}_{5}=(3 \widehat{\delta})^{-1 / 2}+\widehat{C}_{1}\left(\widehat{C}_{*}\right)^{-1 / 2}+\widehat{C}_{6}+\widehat{C}_{4}\left(\widehat{C}_{*}\right)^{-1} .
$$

From (6.8), (7.5), (7.7)-(7.11), and (7.13) it is clear that the constant $\widehat{C}_{5}$ depends only on $\alpha_{0}, \alpha_{1},\|g\|_{L_{\infty}},\left\|g^{-1}\right\|_{L_{\infty}}$, and $r_{0}$. 


\subsection{Estimates in the case $\mathbf{k} \in \widetilde{\Omega},|\mathbf{k}|>\widehat{t_{0}}$}

From (6.7) it follows that

$$
\begin{aligned}
& \left\|\widehat{\mathcal{A}}(\mathbf{k})^{1 / 2} \exp (-\widehat{\mathcal{A}}(\mathbf{k}) \tau)\right\|_{L_{2}(\Omega) \rightarrow L_{2}(\Omega)} \leq \sup \left\{\lambda^{1 / 2} e^{-\lambda \tau}: \lambda \geq \widehat{c}_{*} \widehat{t}_{0}^{2}\right\} \leq \tau^{-1}\left(\widehat{c}_{*}\right)^{-1 / 2}\left(\widehat{t}_{0}\right)^{-1}, \\
& \tau>0, \quad \mathbf{k} \in \widetilde{\Omega}, \quad|\mathbf{k}|>\widehat{t_{0}} \text {. }
\end{aligned}
$$

Combining (6.17), the identity $b(\mathbf{D}+\mathbf{k}) \widehat{P}=b(\mathbf{k}) \widehat{P}$ and (5.2), we have

$$
\begin{aligned}
& \left\|b(\mathbf{D}+\mathbf{k}) \exp \left(-\widehat{\mathcal{A}}^{0}(\mathbf{k}) \tau\right) \widehat{P}\right\|_{L_{2}(\Omega) \rightarrow L_{2}(\Omega)} \leq \alpha_{1}^{1 / 2}|\mathbf{k}| \exp \left(-\widehat{c}_{*}|\mathbf{k}|^{2} \tau\right) \\
& \quad \leq \tau^{-1} \alpha_{1}^{1 / 2}\left(\widehat{c}_{*}\right)^{-1}\left(\widehat{t}_{0}\right)^{-1}, \quad \tau>0, \quad \mathbf{k} \in \widetilde{\Omega}, \quad|\mathbf{k}|>\widehat{t}_{0}
\end{aligned}
$$

Consequently,

$$
\begin{aligned}
& \left\|\widehat{\mathcal{A}}(\mathbf{k})^{1 / 2} \exp \left(-\widehat{\mathcal{A}}^{0}(\mathbf{k}) \tau\right) \widehat{P}\right\|_{L_{2}(\Omega) \rightarrow L_{2}(\Omega)}=\left\|g^{1 / 2} b(\mathbf{D}+\mathbf{k}) \exp \left(-\widehat{\mathcal{A}}^{0}(\mathbf{k}) \tau\right) \widehat{P}\right\|_{L_{2}(\Omega) \rightarrow L_{2}(\Omega)} \\
& \quad \leq \tau^{-1}\|g\|_{L_{\infty}}^{1 / 2} \alpha_{1}^{1 / 2}\left(\widehat{c}_{*}\right)^{-1}\left(\widehat{t}_{0}\right)^{-1}, \quad \tau>0, \quad \mathbf{k} \in \widetilde{\Omega}, \quad|\mathbf{k}|>\widehat{t_{0}} .
\end{aligned}
$$

In order to estimate the operator $\widehat{\mathcal{A}}(\mathbf{k})^{1 / 2} \Lambda b(\mathbf{D}+\mathbf{k}) e^{-\widehat{\mathcal{A}}^{0}(\mathbf{k}) \tau} \widehat{P}$, we represent it as

$$
\widehat{\mathcal{A}}(\mathbf{k})^{1 / 2} \Lambda b(\mathbf{D}+\mathbf{k}) e^{-\widehat{\mathcal{A}}^{0}(\mathbf{k}) \tau} \widehat{P}=\left(\widehat{\mathcal{A}}(\mathbf{k})^{1 / 2} \Lambda \widehat{P}_{m}\right)\left(b(\mathbf{D}+\mathbf{k}) e^{-\widehat{\mathcal{A}}^{0}(\mathbf{k}) \tau} \widehat{P}\right) .
$$

Here $\widehat{P}_{m}$ is the orthogonal projection of $\mathfrak{H}_{*}=L_{2}\left(\Omega ; \mathbb{C}^{m}\right)$ onto the subspace of constants. According to $[\mathrm{BSu} 5,(6.22)]$, we have

$$
\left\|\widehat{\mathcal{A}}(\mathbf{k})^{1 / 2} \Lambda \widehat{P}_{m}\right\|_{L_{2}(\Omega) \rightarrow L_{2}(\Omega)} \leq \mathcal{C}_{\Lambda}:=m^{1 / 2}\|g\|_{L_{\infty}}^{1 / 2}\left(1+r_{1}\left(2 r_{0}\right)^{-1} \alpha_{1}^{1 / 2} \alpha_{0}^{-1 / 2}\|g\|_{L_{\infty}}^{1 / 2}\left\|g^{-1}\right\|_{L_{\infty}}^{1 / 2}\right) .
$$

Here $2 r_{1}=\operatorname{diam} \widetilde{\Omega}$. From $(7.15),(7.17)$, and (7.18) it follows that

$$
\begin{array}{r}
\left\|\widehat{\mathcal{A}}(\mathbf{k})^{1 / 2} \Lambda b(\mathbf{D}+\mathbf{k}) e^{-\widehat{\mathcal{A}}^{0}(\mathbf{k}) \tau} \widehat{P}\right\|_{L_{2}(\Omega) \rightarrow L_{2}(\Omega)} \\
\leq \tau^{-1} \alpha_{1}^{1 / 2}\left(\widehat{c}_{*}\right)^{-1}\left(\widehat{t}_{0}\right)^{-1} \mathcal{C}_{\Lambda} \\
\tau>0, \quad \mathbf{k} \in \widetilde{\Omega}, \quad|\mathbf{k}|>\widehat{t}_{0}
\end{array}
$$

Combining this with (7.14), (7.16), and (7.19), we arrive at the estimate

$$
\begin{array}{r}
\left\|\widehat{\mathcal{A}}(\mathbf{k})^{1 / 2}\left(e^{-\widehat{\mathcal{A}}(\mathbf{k}) \tau}-(I+\Lambda b(\mathbf{D}+\mathbf{k})) e^{-\widehat{\mathcal{A}}^{0}(\mathbf{k}) \tau} \widehat{P}\right)\right\|_{L_{2}(\Omega) \rightarrow L_{2}(\Omega)} \leq \widehat{C}_{7} \tau^{-1} \\
\tau>0, \quad \mathbf{k} \in \widetilde{\Omega}, \quad|\mathbf{k}|>\widehat{t}_{0}
\end{array}
$$

Here

$$
\widehat{C}_{7}=\left(\widehat{c}_{*}\right)^{-1 / 2}\left(\widehat{t}_{0}\right)^{-1}+\alpha_{1}^{1 / 2}\left(\widehat{c}_{*}\right)^{-1}\left(\widehat{t}_{0}\right)^{-1}\left(\|g\|_{L_{\infty}}^{1 / 2}+\mathcal{C}_{\Lambda}\right) .
$$

Relations (6.8), (7.6), and (7.18) show that the constant $\widehat{C}_{7}$ depends only on $m, \alpha_{0}, \alpha_{1}$, $\|g\|_{L_{\infty}},\left\|g^{-1}\right\|_{L_{\infty}}, r_{0}$, and $r_{1}$. 


\subsection{Proof of estimate (7.1) for $\tau \geq 1$}

Finally, from (7.12) and (7.20) it follows that

$$
\left\|\widehat{\mathcal{A}}(\mathbf{k})^{1 / 2}\left(e^{-\widehat{\mathcal{A}}(\mathbf{k}) \tau}-(I+\Lambda b(\mathbf{D}+\mathbf{k})) e^{-\widehat{\mathcal{A}}^{0}(\mathbf{k}) \tau} \widehat{P}\right)\right\|_{L_{2}(\Omega) \rightarrow L_{2}(\Omega)} \leq \widetilde{\mathcal{C}}_{1} \tau^{-1}, \quad \tau>0, \quad \mathbf{k} \in \widetilde{\Omega},
$$

where $\widetilde{\mathcal{C}}_{1}=\max \left\{\widehat{C}_{5}, \widehat{C}_{7}\right\}$.

Now we show that in the principle term of approximation in $(7.21) \widehat{P}$ can be replaced by $I$; only the constant in the remainder estimate will change under such replacement. Using the Fourier series representation for the operator $b(\mathbf{D}+\mathbf{k}) \exp \left(-\widehat{\mathcal{A}}^{0}(\mathbf{k}) \tau\right)(I-\widehat{P})$, we have:

$$
\begin{aligned}
& \left\|\widehat{\mathcal{A}}(\mathbf{k})^{1 / 2} e^{-\widehat{\mathcal{A}}^{0}(\mathbf{k}) \tau}(I-\widehat{P})\right\|_{L_{2}(\Omega) \rightarrow L_{2}(\Omega)}=\left\|g^{1 / 2} b(\mathbf{D}+\mathbf{k}) e^{-\widehat{\mathcal{A}}^{0}(\mathbf{k}) \tau}(I-\widehat{P})\right\|_{L_{2}(\Omega) \rightarrow L_{2}(\Omega)} \\
& \quad \leq\|g\|_{L_{\infty}}^{1 / 2} \sup _{0 \neq \mathbf{b} \in \widetilde{\Gamma}}\left|b(\mathbf{b}+\mathbf{k}) \exp \left(-b(\mathbf{b}+\mathbf{k})^{*} g^{0} b(\mathbf{b}+\mathbf{k}) \tau\right)\right| .
\end{aligned}
$$

By (5.2), (6.16), and the estimate $|\mathbf{b}+\mathbf{k}| \geq r_{0}$ for $\mathbf{k} \in \widetilde{\Omega}$ and $0 \neq \mathbf{b} \in \widetilde{\Gamma}$, we obtain

$$
\begin{aligned}
& \left\|\widehat{\mathcal{A}}(\mathbf{k})^{1 / 2} e^{-\widehat{\mathcal{A}}^{0}(\mathbf{k}) \tau}(I-\widehat{P})\right\|_{L_{2}(\Omega) \rightarrow L_{2}(\Omega)} \leq\|g\|_{L_{\infty}}^{1 / 2} \alpha_{1}^{1 / 2} \sup _{\substack{0 \neq \mathbf{b} \in \widetilde{\Gamma}\\
}}|\mathbf{b}+\mathbf{k}| \exp \left(-\widehat{c}_{*}|\mathbf{b}+\mathbf{k}|^{2} \tau\right) \\
& \quad \leq \tau^{-1}\|g\|_{L_{\infty}}^{1 / 2} \alpha_{1}^{1 / 2}\left(\widehat{c}_{*}\right)^{-1} r_{0}^{-1}, \quad \tau>0, \quad \mathbf{k} \in \widetilde{\Omega} .
\end{aligned}
$$

From (7.21) and (7.23) it follows that

$$
\left\|\widehat{\mathcal{A}}(\mathbf{k})^{1 / 2}\left(e^{-\widehat{\mathcal{A}}(\mathbf{k}) \tau}-(I+\Lambda b(\mathbf{D}+\mathbf{k}) \widehat{P}) e^{-\widehat{\mathcal{A}}^{0}(\mathbf{k}) \tau}\right)\right\|_{L_{2}(\Omega) \rightarrow L_{2}(\Omega)} \leq \mathcal{C}_{1} \tau^{-1}, \quad \tau>0, \quad \mathbf{k} \in \widetilde{\Omega}
$$

where

$$
\mathcal{C}_{1}=\widetilde{\mathcal{C}}_{1}+\|g\|_{L_{\infty}}^{1 / 2} \alpha_{1}^{1 / 2}\left(\widehat{c}_{*}\right)^{-1} r_{0}^{-1} .
$$

Thus, (7.1) is proved in the case where $\tau \geq 1$.

\subsection{Proof of estimate (7.1) for $0<\tau<1$}

If $0<\tau<1$, we estimate each term under the norm sign in (7.1) separately. We have:

$$
\left\|\widehat{\mathcal{A}}(\mathbf{k})^{1 / 2} \exp (-\widehat{\mathcal{A}}(\mathbf{k}) \tau)\right\|_{L_{2}(\Omega) \rightarrow L_{2}(\Omega)} \leq \sup _{\lambda \geq 0} \lambda^{1 / 2} e^{-\lambda \tau} \leq \tau^{-1 / 2}, \quad \tau>0, \quad \mathbf{k} \in \widetilde{\Omega} .
$$

Next, similarly to (7.23), we obtain

$$
\begin{aligned}
& \left\|b(\mathbf{D}+\mathbf{k}) \exp \left(-\widehat{\mathcal{A}}^{0}(\mathbf{k}) \tau\right)\right\|_{L_{2}(\Omega) \rightarrow L_{2}(\Omega)} \leq \alpha_{1}^{1 / 2} \sup _{\mathbf{b} \in \widetilde{\Gamma}}|\mathbf{b}+\mathbf{k}| \exp \left(-\widehat{c}_{*}|\mathbf{b}+\mathbf{k}|^{2} \tau\right) \\
& \quad \leq \tau^{-1 / 2} \alpha_{1}^{1 / 2}\left(\widehat{c}_{*}\right)^{-1 / 2}, \quad \tau>0, \quad \mathbf{k} \in \widetilde{\Omega} .
\end{aligned}
$$


Consequently,

$$
\begin{aligned}
& \left\|\widehat{\mathcal{A}}(\mathbf{k})^{1 / 2} e^{-\widehat{\mathcal{A}}^{0}(\mathbf{k}) \tau}\right\|_{L_{2}(\Omega) \rightarrow L_{2}(\Omega)}=\left\|g^{1 / 2} b(\mathbf{D}+\mathbf{k}) e^{-\widehat{\mathcal{A}}^{0}(\mathbf{k}) \tau}\right\|_{L_{2}(\Omega) \rightarrow L_{2}(\Omega)} \\
& \quad \leq \tau^{-1 / 2}\|g\|_{L_{\infty}}^{1 / 2} \alpha_{1}^{1 / 2}\left(\widehat{c}_{*}\right)^{-1 / 2}, \quad \tau>0, \quad \mathbf{k} \in \widetilde{\Omega} .
\end{aligned}
$$

Now, from (7.18) and (7.26) it follows that

$$
\left\|\widehat{\mathcal{A}}(\mathbf{k})^{1 / 2} \Lambda b(\mathbf{D}+\mathbf{k}) e^{-\widehat{\mathcal{A}}^{0}(\mathbf{k}) \tau} \widehat{P}\right\|_{L_{2}(\Omega) \rightarrow L_{2}(\Omega)} \leq \tau^{-1 / 2} \mathcal{C}_{\Lambda} \alpha_{1}^{1 / 2}\left(\widehat{c}_{*}\right)^{-1 / 2}, \quad \tau>0, \quad \mathbf{k} \in \widetilde{\Omega}
$$

Relations (7.25), (7.27), and (7.28) imply estimate (7.1) for $0<\tau<1$ with

$$
\mathcal{C}_{2}=1+\alpha_{1}^{1 / 2}\left(\widehat{c}_{*}\right)^{-1 / 2}\left(\|g\|_{L_{\infty}}^{1 / 2}+\mathcal{C}_{\Lambda}\right) .
$$

This completes the proof of Theorem 7.1.

\subsection{Approximation of the operator $g b(\mathbf{D}+\mathbf{k}) \exp (-\widehat{\mathcal{A}}(\mathbf{k}) \tau)$}

Besides Theorem 7.1 we need the following statement.

Theorem 7.2. Suppose that the assumptions of Theorem 7.1 are satisfied. Let $\widetilde{g}(\mathbf{x})$ be the matrix (6.12). Then for $\tau>0$ and $\mathbf{k} \in \widetilde{\Omega}$ we have

$$
\begin{gathered}
\left\|g b(\mathbf{D}+\mathbf{k}) e^{-\widehat{\mathcal{A}}(\mathbf{k}) \tau}-\widetilde{g} b(\mathbf{D}+\mathbf{k}) e^{-\widehat{\mathcal{A}}^{0}(\mathbf{k}) \tau} \widehat{P}\right\|_{L_{2}(\Omega) \rightarrow L_{2}(\Omega)} \leq R_{1}(\tau), \\
R_{1}(\tau)=\left\{\begin{array}{c}
\mathcal{C}_{3} \tau^{-1}, \quad \tau \geq 1 \\
\mathcal{C}_{4} \tau^{-1 / 2}, \quad 0<\tau<1 .
\end{array}\right.
\end{gathered}
$$

The constants $\mathcal{C}_{3}$ and $\mathcal{C}_{4}$ are defined below in (7.40); they depend only on $d, m, \alpha_{0}, \alpha_{1}$, $\|g\|_{L_{\infty}},\left\|g^{-1}\right\|_{L_{\infty}}$, and the parameters of the lattice $\Gamma$.

Proof. Estimate (7.21) can be rewritten as

$$
\left\|g^{1 / 2} b(\mathbf{D}+\mathbf{k})\left(e^{-\widehat{\mathcal{A}}(\mathbf{k}) \tau}-(I+\Lambda b(\mathbf{D}+\mathbf{k})) e^{-\widehat{\mathcal{A}}^{0}(\mathbf{k}) \tau} \widehat{P}\right)\right\|_{L_{2}(\Omega) \rightarrow L_{2}(\Omega)} \leq \widetilde{\mathcal{C}}_{1} \tau^{-1}, \quad \tau>0, \quad \mathbf{k} \in \widetilde{\Omega}
$$

From the other side, by (7.25), (7.27), and (7.28), we have

$\left\|g^{1 / 2} b(\mathbf{D}+\mathbf{k})\left(e^{-\widehat{\mathcal{A}}(\mathbf{k}) \tau}-(I+\Lambda b(\mathbf{D}+\mathbf{k})) e^{-\widehat{\mathcal{A}}^{0}(\mathbf{k}) \tau} \widehat{P}\right)\right\|_{L_{2}(\Omega) \rightarrow L_{2}(\Omega)} \leq \mathcal{C}_{2} \tau^{-1 / 2}, \quad \tau>0, \quad \mathbf{k} \in \widetilde{\Omega}$

Applying (7.32) for $\tau \geq 1$ and (7.33) for $0<\tau<1$, we obtain

$$
\begin{gathered}
\left\|g b(\mathbf{D}+\mathbf{k})\left(e^{-\widehat{\mathcal{A}}(\mathbf{k}) \tau}-(I+\Lambda b(\mathbf{D}+\mathbf{k})) e^{-\widehat{\mathcal{A}}^{0}(\mathbf{k}) \tau} \widehat{P}\right)\right\|_{L_{2}(\Omega) \rightarrow L_{2}(\Omega)} \leq \widetilde{R}_{1}(\tau), \quad \tau>0, \quad \mathbf{k} \in \widetilde{\Omega} \\
\widetilde{R}_{1}(\tau)=\left\{\begin{array}{c}
\widetilde{\mathcal{C}}_{1}\|g\|_{L_{\infty}}^{1 / 2} \tau^{-1}, \quad \tau \geq 1 \\
\mathcal{C}_{2}\|g\|_{L_{\infty}}^{1 / 2} \tau^{-1 / 2}, \quad 0<\tau<1 .
\end{array}\right.
\end{gathered}
$$


Now we transform the term with $\Lambda$ in (7.34). By (5.3), we have

$$
b(\mathbf{D}+\mathbf{k})(\Lambda \mathbf{u})=(b(\mathbf{D}) \Lambda) \mathbf{u}+\sum_{j=1}^{d} b_{j} \Lambda\left(D_{j}+k_{j}\right) \mathbf{u} .
$$

Consequently,

$$
\begin{aligned}
& g b(\mathbf{D}+\mathbf{k})(I+\Lambda b(\mathbf{D}+\mathbf{k})) e^{-\widehat{\mathcal{A}}^{0}(\mathbf{k}) \tau} \widehat{P}=g\left(\mathbf{1}_{m}+(b(\mathbf{D}) \Lambda)\right) b(\mathbf{D}+\mathbf{k}) e^{-\widehat{\mathcal{A}}^{0}(\mathbf{k}) \tau} \widehat{P} \\
& +g \sum_{j=1}^{d} b_{j} \Lambda\left(D_{j}+k_{j}\right) b(\mathbf{D}+\mathbf{k}) e^{-\widehat{\mathcal{A}}^{0}(\mathbf{k}) \tau} \widehat{P} .
\end{aligned}
$$

Let us show that the second term in the right-hand side of (7.35) can be viewed as an error term. Taking (5.3) into account, we have

$$
\begin{aligned}
& \left\|g \sum_{j=1}^{d} b_{j} \Lambda\left(D_{j}+k_{j}\right) b(\mathbf{D}+\mathbf{k}) e^{-\widehat{\mathcal{A}}^{0}(\mathbf{k}) \tau} \widehat{P}\right\|_{L_{2}(\Omega) \rightarrow L_{2}(\Omega)} \\
& \quad \leq \sum_{j=1}^{d}\|g\|_{L_{\infty}} \alpha_{1}^{1 / 2}\left\|\Lambda \widehat{P}_{m}\right\|_{L_{2}(\Omega) \rightarrow L_{2}(\Omega)}\left\|\left(D_{j}+k_{j}\right) b(\mathbf{D}+\mathbf{k}) e^{-\widehat{\mathcal{A}}^{0}(\mathbf{k}) \tau} \widehat{P}\right\|_{L_{2}(\Omega) \rightarrow L_{2}(\Omega)} .
\end{aligned}
$$

Next,

$$
\left\|\Lambda \widehat{P}_{m}\right\|_{L_{2}(\Omega) \rightarrow L_{2}(\Omega)}=|\Omega|^{-1 / 2}\|\Lambda\|_{L_{2}(\Omega)} \leq m^{1 / 2}\left(2 r_{0}\right)^{-1} \alpha_{0}^{-1 / 2}\|g\|_{L_{\infty}}^{1 / 2}\left\|g^{-1}\right\|_{L_{\infty}}^{1 / 2} .
$$

Here we have used an estimate for $\|\Lambda\|_{L_{2}(\Omega)}$ obtained in [BSu4, Subsec. 7.3]. Due to the presence of the projection $\widehat{P}$, we have

$$
\begin{aligned}
& \left\|\left(D_{j}+k_{j}\right) b(\mathbf{D}+\mathbf{k}) e^{-\widehat{\mathcal{A}}^{0}(\mathbf{k}) \tau} \widehat{P}\right\|_{L_{2}(\Omega) \rightarrow L_{2}(\Omega)}=\left|k_{j} b(\mathbf{k}) \exp \left(-b(\mathbf{k})^{*} g^{0} b(\mathbf{k}) \tau\right)\right| \\
& \quad \leq \alpha_{1}^{1 / 2}|\mathbf{k}|^{2} \exp \left(-\widehat{c}_{*}|\mathbf{k}|^{2} \tau\right), \quad \tau>0, \quad \mathbf{k} \in \widetilde{\Omega}
\end{aligned}
$$

We have taken (5.2) and (6.16) into account. For $\tau \geq 1$ we estimate the term $|\mathbf{k}|^{2} e^{-\widehat{c}_{*}|\mathbf{k}|^{2} \tau}$ by $\tau^{-1}\left(\widehat{c}_{*}\right)^{-1}$, while for $0<\tau<1$ we estimate it by $\tau^{-1 / 2}\left(\widehat{c}_{*}\right)^{-1 / 2} r_{1}$. As a result, from $(7.36)-(7.38)$ it follows that

$$
\begin{gathered}
\left\|g \sum_{j=1}^{d} b_{j} \Lambda\left(D_{j}+k_{j}\right) b(\mathbf{D}+\mathbf{k}) e^{-\widehat{\mathcal{A}}^{0}(\mathbf{k}) \tau} \widehat{P}\right\|_{L_{2}(\Omega) \rightarrow L_{2}(\Omega)} \leq d m^{1 / 2}\left(2 r_{0}\right)^{-1} \alpha_{0}^{-1 / 2} \alpha_{1}\|g\|_{L_{\infty}}^{3 / 2}\left\|g^{-1}\right\|_{L_{\infty}}^{1 / 2} \rho(\tau), \\
\rho(\tau)=\left\{\begin{array}{cc}
\left(\widehat{c}_{*}\right)^{-1} \tau^{-1}, & \tau \geq 1, \\
\left(\widehat{c}_{*}\right)^{-1 / 2} r_{1} \tau^{-1 / 2}, & 0<\tau<1 .
\end{array}\right.
\end{gathered}
$$

Recall the notation $\widetilde{g}=g\left(\mathbf{1}_{m}+b(\mathbf{D}) \Lambda\right)$ (see (6.12)). Now, relations (7.34), (7.35), and (7.39) imply estimate (7.30); the constants $\mathcal{C}_{3}$ and $\mathcal{C}_{4}$ in (7.31) are given by

$$
\begin{aligned}
& \mathcal{C}_{3}=\widetilde{\mathcal{C}}_{1}\|g\|_{L_{\infty}}^{1 / 2}+d m^{1 / 2}\left(2 r_{0}\right)^{-1} \alpha_{0}^{-1 / 2} \alpha_{1}\|g\|_{L_{\infty}}^{3 / 2}\left\|g^{-1}\right\|_{L_{\infty}}^{1 / 2}\left(\widehat{c}_{*}\right)^{-1} \\
& \mathcal{C}_{4}=\mathcal{C}_{2}\|g\|_{L_{\infty}}^{1 / 2}+d m^{1 / 2}\left(2 r_{0}\right)^{-1} \alpha_{0}^{-1 / 2} \alpha_{1}\|g\|_{L_{\infty}}^{3 / 2}\left\|g^{-1}\right\|_{L_{\infty}}^{1 / 2}\left(\widehat{c}_{*}\right)^{-1 / 2} r_{1} .
\end{aligned}
$$




\subsection{Special cases}

Under the condition

$$
\Lambda(\mathbf{x}) b(\boldsymbol{\theta})=0, \quad \mathbf{x} \in \Omega, \quad \boldsymbol{\theta} \in \mathbb{S}^{d-1},
$$

we have $\widehat{Z}(\boldsymbol{\theta})=0$. Then the corrector in (7.1) is equal to zero. In particular, (7.41) is satisfied if $g^{0}=\bar{g}$, i. e., under condition (6.19); $\Lambda(\mathbf{x})=0$ in this case.

Under condition (7.41), the constants in estimate (7.1) can be made sharper. Indeed, in this case estimate (7.12) takes the form

$$
\left\|\widehat{\mathcal{A}}(\mathbf{k})^{1 / 2}\left(e^{-\widehat{\mathcal{A}}(\mathbf{k}) \tau}-e^{-\widehat{\mathcal{A}}^{0}(\mathbf{k}) \tau} \widehat{P}\right)\right\|_{L_{2}(\Omega) \rightarrow L_{2}(\Omega)} \leq \widehat{C}_{5} \tau^{-1}, \quad \tau>0, \quad|\mathbf{k}| \leq \widehat{t}_{0}
$$

For $\mathbf{k} \in \widetilde{\Omega}$ and $|\mathbf{k}|>\widehat{t}_{0}$ we use estimates (7.14) and (7.16). Hence,

$$
\left\|\widehat{\mathcal{A}}(\mathbf{k})^{1 / 2}\left(e^{-\widehat{\mathcal{A}}(\mathbf{k}) \tau}-e^{-\widehat{\mathcal{A}}^{0}(\mathbf{k}) \tau} \widehat{P}\right)\right\|_{L_{2}(\Omega) \rightarrow L_{2}(\Omega)} \leq \widetilde{\mathcal{C}}_{1}^{0} \tau^{-1}, \quad \tau>0, \quad \mathbf{k} \in \widetilde{\Omega},
$$

where

$$
\widetilde{\mathcal{C}}_{1}^{0}=\max \left\{\widehat{C}_{5},\left(\widehat{c}_{*}\right)^{-1 / 2}\left(\widehat{t}_{0}\right)^{-1}+\|g\|_{L_{\infty}}^{1 / 2} \alpha_{1}^{1 / 2}\left(\widehat{c}_{*}\right)^{-1}\left(\widehat{t}_{0}\right)^{-1}\right\} .
$$

We also take estimate (7.23) into account, and for $0<\tau<1$ apply inequalities (7.25) and (7.27). As a result, we arrive at the following statement.

Proposition 7.3. Suppose that the assumptions of Theorem 7.1 and condition (7.41) are satisfied. Then for $\tau>0$ and $\mathbf{k} \in \widetilde{\Omega}$ we have

$$
\begin{gathered}
\left\|\widehat{\mathcal{A}}(\mathbf{k})^{1 / 2}\left(e^{-\widehat{\mathcal{A}}(\mathbf{k}) \tau}-e^{-\widehat{\mathcal{A}}^{0}(\mathbf{k}) \tau}\right)\right\|_{L_{2}(\Omega) \rightarrow L_{2}(\Omega)} \leq \Phi_{1}^{0}(\tau), \\
\Phi_{1}^{0}(\tau)=\left\{\begin{array}{c}
\mathcal{C}_{1}^{0} \tau^{-1}, \quad \tau \geq 1, \\
\mathcal{C}_{2}^{0} \tau^{-1 / 2}, \quad 0<\tau<1 .
\end{array}\right.
\end{gathered}
$$

The constants $\mathcal{C}_{1}^{0}$ and $\mathcal{C}_{2}^{0}$ are given by

$$
\mathcal{C}_{1}^{0}=\widetilde{\mathcal{C}}_{1}^{0}+\|g\|_{L_{\infty}}^{1 / 2} \alpha_{1}^{1 / 2}\left(\widehat{c}_{*}\right)^{-1} r_{0}^{-1}, \quad \mathcal{C}_{2}^{0}=1+\|g\|_{L_{\infty}}^{1 / 2} \alpha_{1}^{1 / 2}\left(\widehat{c}_{*}\right)^{-1 / 2}
$$

they depend only on $\alpha_{0}, \alpha_{1},\|g\|_{L_{\infty}},\left\|g^{-1}\right\|_{L_{\infty}}$, and the parameters of the lattice $\Gamma$.

Finally, we consider a special case in approximation (7.30). Suppose that condition (6.20) is satisfied. In this case $\widetilde{g}(\mathbf{x})=g=g^{0}$. Then for $\tau \geq 1$ inequality (7.30) takes the form

$$
\left\|g b(\mathbf{D}+\mathbf{k}) e^{-\widehat{\mathcal{A}}(\mathbf{k}) \tau}-g^{0} b(\mathbf{D}+\mathbf{k}) e^{-\widehat{\mathcal{A}}^{0}(\mathbf{k}) \tau} \widehat{P}\right\|_{L_{2}(\Omega) \rightarrow L_{2}(\Omega)} \leq \mathcal{C}_{3} \tau^{-1}, \quad \tau \geq 1, \quad \mathbf{k} \in \widetilde{\Omega} .
$$

Here $\widehat{P}$ can be replaced by $I$. Indeed, similarly to (7.22) and (7.23), we have

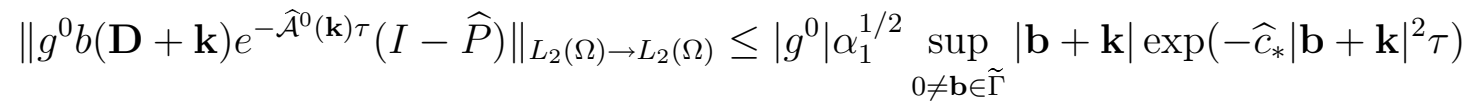

$$
\begin{aligned}
& \leq \tau^{-1}\|g\|_{L_{\infty}} \alpha_{1}^{1 / 2}\left(\widehat{c}_{*}\right)^{-1} r_{0}^{-1}, \quad \tau>0, \quad \mathbf{k} \in \widetilde{\Omega} .
\end{aligned}
$$


For $0<\tau<1$ we apply the estimate which follows from (7.25) and (7.26):

$$
\left\|g b(\mathbf{D}+\mathbf{k}) e^{-\widehat{\mathcal{A}}(\mathbf{k}) \tau}-g^{0} b(\mathbf{D}+\mathbf{k}) e^{-\widehat{\mathcal{A}}^{0}(\mathbf{k}) \tau}\right\|_{L_{2}(\Omega) \rightarrow L_{2}(\Omega)} \leq \mathcal{C}_{4}^{0} \tau^{-1 / 2}, \quad \tau>0, \quad \mathbf{k} \in \widetilde{\Omega} .
$$

Here

$$
\mathcal{C}_{4}^{0}=\|g\|_{L_{\infty}}^{1 / 2}+\|g\|_{L_{\infty}} \alpha_{1}^{1 / 2}\left(\widehat{c}_{*}\right)^{-1 / 2} .
$$

As a result, relations (7.43)-(7.45) imply the following statement.

Proposition 7.4. Suppose that the assumptions of Theorem 7.2 and condition (6.20) are satisfied. Then for $\tau>0$ and $\mathbf{k} \in \widetilde{\Omega}$ we have

$$
\begin{gathered}
\left\|g b(\mathbf{D}+\mathbf{k}) e^{-\widehat{\mathcal{A}}(\mathbf{k}) \tau}-g^{0} b(\mathbf{D}+\mathbf{k}) e^{-\widehat{\mathcal{A}}^{0}(\mathbf{k}) \tau}\right\|_{L_{2}(\Omega) \rightarrow L_{2}(\Omega)} \leq R_{1}^{0}(\tau) \\
R_{1}^{0}(\tau)=\left\{\begin{array}{c}
\mathcal{C}_{3}^{0} \tau^{-1}, \quad \tau \geq 1 \\
\mathcal{C}_{4}^{0} \tau^{-1 / 2}, \quad 0<\tau<1 .
\end{array}\right.
\end{gathered}
$$

The constant $\mathcal{C}_{3}^{0}$ is given by $\mathcal{C}_{3}^{0}=\mathcal{C}_{3}+\|g\|_{L_{\infty}} \alpha_{1}^{1 / 2}\left(\widehat{C}_{*}\right)^{-1} r_{0}^{-1}$, and $\mathcal{C}_{4}^{0}$ is defined by (7.46). The constant $\mathcal{C}_{4}^{0}$ depends only on $\alpha_{0}, \alpha_{1},\|g\|_{L_{\infty}},\left\|g^{-1}\right\|_{L_{\infty}} ; \mathcal{C}_{3}^{0}$ depends on the same parameters and on $d, m$, and the parameters of the lattice $\Gamma$.

\section{Approximation of the operator $f \exp (-\mathcal{A}(\mathbf{k}) \tau) f^{*}$}

\subsection{Incorporation of $\mathcal{A}(\mathrm{k})$ in the framework of Section 4}

Now we consider the operator $\operatorname{exponential~} \exp (-\mathcal{A}(\mathbf{k}) \tau)$ for the operator

$$
\mathcal{A}(\mathbf{k})=f(\mathbf{x})^{*} b(\mathbf{D}+\mathbf{k})^{*} g(\mathbf{x}) b(\mathbf{D}+\mathbf{k}) f(\mathbf{x})
$$

(with periodic boundary conditions) in the general case $f \neq \mathbf{1}_{n}$. Applying Theorem 4.1, we shall obtain approximation of the operator $f \exp (-\mathcal{A}(\mathbf{k}) \tau) f^{*}$.

Now the assumptions of Subsec. 4.1 are satisfied with $\mathfrak{H}=\widehat{\mathfrak{H}}=L_{2}\left(\Omega ; \mathbb{C}^{n}\right)$ and $\mathfrak{H}_{*}=$ $L_{2}\left(\Omega ; \mathbb{C}^{m}\right)$. The role of $\widehat{A}(t)$ is played by the operator $\widehat{A}(t ; \boldsymbol{\theta})=\widehat{\mathcal{A}}(t \boldsymbol{\theta})$, and the role of $A(t)$ is played by $A(t ; \boldsymbol{\theta})=\mathcal{A}(t \boldsymbol{\theta})$. An isomorphism $M$ is the operator of multiplication by the function $f(\mathbf{x})$. Relation (4.2) corresponds to the identity $\mathcal{A}(\mathbf{k})=f^{*} \widehat{\mathcal{A}}(\mathbf{k}) f$.

Next, the operator $Q$ (see (4.3)) is the operator of multiplication by the matrix-valued function $Q(\mathbf{x})=\left(f(\mathbf{x}) f(\mathbf{x})^{*}\right)^{-1}$. The block $Q_{\widehat{\mathfrak{N}}}$ of $Q$ in the subspace $\widehat{\mathfrak{N}}$ (see (6.5)) is the operator of multiplication by the constant matrix

$$
\bar{Q}=\left(\underline{f f^{*}}\right)^{-1}=|\Omega|^{-1} \int_{\Omega}\left(f(\mathbf{x}) f(\mathbf{x})^{*}\right)^{-1} d \mathbf{x} .
$$

The operator $M_{0}($ see $(4.10))$ acts in $\widehat{\mathfrak{N}}$ as multiplication by the matrix

$$
f_{0}=(\bar{Q})^{-1 / 2}=\left(\underline{f f^{*}}\right)^{1 / 2} .
$$


Obviously,

$$
\left|f_{0}\right| \leq\|f\|_{L_{\infty}}, \quad\left|\left(f_{0}\right)^{-1}\right| \leq\left\|f^{-1}\right\|_{L_{\infty}} .
$$

Now we specify the operators from (4.12). By (6.10), we have

$$
t^{2} M_{0} \widehat{S}(\boldsymbol{\theta}) M_{0}=f_{0} b(\mathbf{k})^{*} g^{0} b(\mathbf{k}) f_{0} .
$$

Let $\mathcal{A}^{0}$ be the operator in $L_{2}\left(\mathbb{R}^{d} ; \mathbb{C}^{n}\right)$ given by the expression

$$
\mathcal{A}^{0}=f_{0} b(\mathbf{D})^{*} g^{0} b(\mathbf{D}) f_{0} .
$$

Let $\mathcal{A}^{0}(\mathbf{k})$ be the corresponding operator family in $L_{2}\left(\Omega ; \mathbb{C}^{n}\right)$ given by the expression

$$
\mathcal{A}^{0}(\mathbf{k})=f_{0} b(\mathbf{D}+\mathbf{k})^{*} g^{0} b(\mathbf{D}+\mathbf{k}) f_{0},
$$

with periodic boundary conditions. By (6.16), (8.3), and the identity $c_{*}=\widehat{c}_{*}\left\|f^{-1}\right\|_{L_{\infty}}^{-2}$, the symbol of the operator $\mathcal{A}^{0}$ satisfies the estimate

$$
f_{0} b(\mathbf{k})^{*} g^{0} b(\mathbf{k}) f_{0} \geq c_{*}|\mathbf{k}|^{2} \mathbf{1}_{n}, \quad \mathbf{k} \in \mathbb{R}^{d} .
$$

Hence, using the Fourier series representation for the operator $\mathcal{A}^{0}(\mathbf{k})$ and (5.10), we obtain

$$
\mathcal{A}^{0}(\mathbf{k}) \geq c_{*}|\mathbf{k}|^{2} I, \quad \mathbf{k} \in \widetilde{\Omega} .
$$

By (8.4), (8.5), and (6.6), we have $t^{2} M_{0} \widehat{S}(\boldsymbol{\theta}) M_{0} \widehat{P}=\mathcal{A}^{0}(\mathbf{k}) \widehat{P}$, whence

$$
M_{0} e^{-t^{2} M_{0} \widehat{S}(\boldsymbol{\theta}) M_{0} \tau} M_{0} \widehat{P}=f_{0} e^{-\mathcal{A}^{0}(\mathbf{k}) \tau} f_{0} \widehat{P}
$$

According to $[\mathrm{BSu} 4, \S 5]$, the role of $\widehat{Z}_{Q}$ is played by the operator

$$
\widehat{Z}_{Q}(\boldsymbol{\theta})=\Lambda_{Q} b(\boldsymbol{\theta}) \widehat{P} .
$$

Here $\Lambda_{Q}$ is the operator of multiplication by the $\Gamma$-periodic $(n \times m)$-matrix-valued function $\Lambda_{Q}(\mathbf{x})$, which is the solution of the problem

$$
b(\mathbf{D})^{*} g(\mathbf{x})\left(b(\mathbf{D}) \Lambda_{Q}(\mathbf{x})+\mathbf{1}_{m}\right)=0, \quad \int_{\Omega} Q(\mathbf{x}) \Lambda_{Q}(\mathbf{x}) d \mathbf{x}=0
$$

Note that

$$
\Lambda_{Q}(\mathbf{x})=\Lambda(\mathbf{x})+\Lambda_{Q}^{0}, \quad \Lambda_{Q}^{0}=-(\bar{Q})^{-1}(\overline{Q \Lambda}),
$$

where $\Lambda$ is the $\Gamma$-periodic solution of problem (6.11). From (8.9) due to the presence of the projection $\widehat{P}$ it follows that

$$
t \widehat{Z}_{Q}(\boldsymbol{\theta}) \widehat{P}=\Lambda_{Q} b(\mathbf{k}) \widehat{P}=\Lambda_{Q} b(\mathbf{D}+\mathbf{k}) \widehat{P} .
$$




\subsection{Estimates in the case $|\mathbf{k}| \leq t_{0}$}

In order to approximate the operator $f \exp (-\mathcal{A}(\mathbf{k}) \tau) f^{*}$, we apply Theorem 4.1. We only need to specify the constants in estimates. The constants $c_{*}, \delta$ and $t_{0}$ are defined by (5.17), (6.2), and (6.3). Using the estimate $\left\|X_{1}(\boldsymbol{\theta})\right\| \leq \alpha_{1}^{1 / 2}\|g\|_{L_{\infty}}^{1 / 2}\|f\|_{L_{\infty}}$, instead of the precise values $C_{1}(\boldsymbol{\theta})=\beta_{1} \delta^{-1 / 2}\left\|X_{1}(\boldsymbol{\theta})\right\|$ and $C_{2}(\boldsymbol{\theta})=\beta_{2} \delta^{-1 / 2}\left\|X_{1}(\boldsymbol{\theta})\right\|^{3}$ (see Subsec. 2.4), we take

$$
\begin{aligned}
& C_{1}=\beta_{1} \delta^{-1 / 2} \alpha_{1}^{1 / 2}\|g\|_{L_{\infty}}^{1 / 2}\|f\|_{L_{\infty}}=2 \beta_{1} r_{0}^{-1} \alpha_{0}^{-1 / 2} \alpha_{1}^{1 / 2}\|g\|_{L_{\infty}}^{1 / 2}\left\|g^{-1}\right\|_{L_{\infty}}^{1 / 2}\|f\|_{L_{\infty}}\left\|f^{-1}\right\|_{L_{\infty}}, \\
& C_{2}=\beta_{2} \delta^{-1 / 2} \alpha_{1}^{3 / 2}\|g\|_{L_{\infty}}^{3 / 2}\|f\|_{L_{\infty}}^{3}=2 \beta_{2} r_{0}^{-1} \alpha_{0}^{-1 / 2} \alpha_{1}^{3 / 2}\|g\|_{L_{\infty}}^{3 / 2}\left\|g^{-1}\right\|_{L_{\infty}}^{1 / 2}\|f\|_{L_{\infty}}^{3}\left\|f^{-1}\right\|_{L_{\infty}} .
\end{aligned}
$$

Similarly, instead of the precise values $C_{3}(\boldsymbol{\theta})=\left(1+\beta_{2}\right)\left\|X_{1}(\boldsymbol{\theta})\right\|^{2}, C_{4}(\boldsymbol{\theta})=\beta_{3} \delta^{-1 / 2}\left\|X_{1}(\boldsymbol{\theta})\right\|^{2}$ (see $(2.17),(2.19))$, we take

$$
\begin{gathered}
C_{3}=\left(1+\beta_{2}\right) \alpha_{1}\|g\|_{L_{\infty}}\|f\|_{L_{\infty}}^{2} \\
C_{4}=\beta_{3} \delta^{-1 / 2} \alpha_{1}\|g\|_{L_{\infty}}\|f\|_{L_{\infty}}^{2}=2 \beta_{3} r_{0}^{-1} \alpha_{0}^{-1 / 2} \alpha_{1}\|g\|_{L_{\infty}}\left\|g^{-1}\right\|_{L_{\infty}}^{1 / 2}\|f\|_{L_{\infty}}^{2}\left\|f^{-1}\right\|_{L_{\infty}} .
\end{gathered}
$$

According to (3.14), we put

$$
C_{6}=C_{3}^{1 / 2} \sup _{\alpha>0}\left(2 C_{1} \alpha+C_{2} \alpha^{2}\right) e^{-c_{*} \alpha},
$$

where $c_{*}$ is defined by (5.17), and $C_{1}, C_{2}, C_{3}$ are defined by (8.12)-(8.14).

By Theorem 4.1, taking (8.8) and (8.11) into account, for $\tau>0$ and $|\mathbf{k}| \leq t_{0}$ we obtain:

$$
\left\|\widehat{\mathcal{A}}(\mathbf{k})^{1 / 2}\left(f e^{-\mathcal{A}(\mathbf{k}) \tau} f^{*}-\left(I+\Lambda_{Q} b(\mathbf{D}+\mathbf{k})\right) f_{0} e^{-\mathcal{A}^{0}(\mathbf{k}) \tau} f_{0} \widehat{P}\right)\right\|_{L_{2}(\Omega) \rightarrow L_{2}(\Omega)} \leq C_{5}\|f\|_{L_{\infty}} \tau^{-1},
$$

where

$$
C_{5}=(3 \delta)^{-1 / 2}+C_{1} c_{*}^{-1 / 2}+C_{6}+C_{4} c_{*}^{-1} .
$$

According to (5.17), (6.2), (8.12)-(8.16), the constant (8.18) depends only on $\alpha_{0}, \alpha_{1},\|g\|_{L_{\infty}}$, $\left\|g^{-1}\right\|_{L_{\infty}},\|f\|_{L_{\infty}},\left\|f^{-1}\right\|_{L_{\infty}}$, and $r_{0}$.

Using (8.10), we show that $\Lambda_{Q}$ can be replaced by $\Lambda$ in (8.17). Only the constant in estimate will change under such replacement. Indeed, due to the presence of the projection $\widehat{P}$, taking (5.2), (8.3), and (8.7) into account, we obtain:

$$
\begin{aligned}
& \left\|\widehat{\mathcal{A}}(\mathbf{k})^{1 / 2} \Lambda_{Q}^{0} b(\mathbf{D}+\mathbf{k}) f_{0} e^{-\mathcal{A}^{0}(\mathbf{k}) \tau} f_{0} \widehat{P}\right\|_{L_{2}(\Omega) \rightarrow L_{2}(\Omega)} \\
& \quad=\left\|g^{1 / 2} b(\mathbf{D}+\mathbf{k}) \Lambda_{Q}^{0} b(\mathbf{D}+\mathbf{k}) f_{0} e^{-\mathcal{A}^{0}(\mathbf{k}) \tau} f_{0} \widehat{P}\right\|_{L_{2}(\Omega) \rightarrow L_{2}(\Omega)} \\
& \quad \leq\|g\|_{L_{\infty}}^{1 / 2}\left\|b(\mathbf{k}) \Lambda_{Q}^{0} b(\mathbf{k}) f_{0} e^{-\mathcal{A}^{0}(\mathbf{k}) \tau} f_{0} \widehat{P}\right\|_{L_{2}(\Omega) \rightarrow L_{2}(\Omega)} \\
& \quad \leq\|g\|_{L_{\infty}}^{1 / 2}\|f\|_{L_{\infty}}^{2}\left|\Lambda_{Q}^{0}\right| \alpha_{1}|\mathbf{k}|^{2} e^{-c_{*}|\mathbf{k}|^{2} \tau} \\
& \quad \leq \tau^{-1}\|g\|_{L_{\infty}}^{1 / 2}\|f\|_{L_{\infty}}^{2}\left|\Lambda_{Q}^{0}\right| \alpha_{1} c_{*}^{-1}, \quad \tau>0, \quad \mathbf{k} \in \widetilde{\Omega}
\end{aligned}
$$

Next, according to $[\mathrm{BSu} 4, \S 7]$, we have

$$
\left|\Lambda_{Q}^{0}\right| \leq m^{1 / 2}\left(2 r_{0}\right)^{-1} \alpha_{0}^{-1 / 2}\|g\|_{L_{\infty}}^{1 / 2}\left\|g^{-1}\right\|_{L_{\infty}}^{1 / 2}\|f\|_{L_{\infty}}^{2}\left\|f^{-1}\right\|_{L_{\infty}}^{2} .
$$


Combining (8.17), (8.19), (8.20), and taking (8.10) and (5.17) into account, we obtain

$$
\begin{array}{r}
\left\|\widehat{\mathcal{A}}(\mathbf{k})^{1 / 2}\left(f e^{-\mathcal{A}(\mathbf{k}) \tau} f^{*}-(I+\Lambda b(\mathbf{D}+\mathbf{k})) f_{0} e^{-\mathcal{A}^{0}(\mathbf{k}) \tau} f_{0} \widehat{P}\right)\right\|_{L_{2}(\Omega) \rightarrow L_{2}(\Omega)} \leq C_{8} \tau^{-1} \\
\tau>0, \quad|\mathbf{k}| \leq t_{0}
\end{array}
$$

where

$$
C_{8}=C_{5}\|f\|_{L_{\infty}}+m^{1 / 2}\left(2 r_{0}\right)^{-1} \alpha_{0}^{-3 / 2} \alpha_{1}\|g\|_{L_{\infty}}\left\|g^{-1}\right\|_{L_{\infty}}^{3 / 2}\|f\|_{L_{\infty}}^{4}\left\|f^{-1}\right\|_{L_{\infty}}^{4}
$$

\subsection{Estimates in the case $\mathbf{k} \in \widetilde{\Omega},|\mathbf{k}|>t_{0}$}

For $|\mathbf{k}|>t_{0}$ each term under the norm sign in (8.21) is estimated separately. We have:

$$
\begin{aligned}
\left\|\widehat{\mathcal{A}}(\mathbf{k})^{1 / 2} f e^{-\mathcal{A}(\mathbf{k}) \tau} f^{*}\right\|_{L_{2}(\Omega) \rightarrow L_{2}(\Omega)} & =\left\|g^{1 / 2} b(\mathbf{D}+\mathbf{k}) f e^{-\mathcal{A}(\mathbf{k}) \tau} f^{*}\right\|_{L_{2}(\Omega) \rightarrow L_{2}(\Omega)} \\
=\left\|\mathcal{A}(\mathbf{k})^{1 / 2} e^{-\mathcal{A}(\mathbf{k}) \tau} f^{*}\right\|_{L_{2}(\Omega) \rightarrow L_{2}(\Omega)} & \leq\|f\|_{L_{\infty}}\left\|\mathcal{A}(\mathbf{k})^{1 / 2} e^{-\mathcal{A}(\mathbf{k}) \tau}\right\|_{L_{2}(\Omega) \rightarrow L_{2}(\Omega)}
\end{aligned}
$$

From (5.16) for $|\mathbf{k}|>t_{0}$ it follows that

$$
\left\|\mathcal{A}(\mathbf{k})^{1 / 2} e^{-\mathcal{A}(\mathbf{k}) \tau}\right\|_{L_{2}(\Omega) \rightarrow L_{2}(\Omega)} \leq \sup _{\lambda \geq c_{*} t_{0}^{2}} \lambda^{1 / 2} e^{-\lambda \tau} \leq \tau^{-1} c_{*}^{-1 / 2} t_{0}^{-1}, \quad \tau>0, \quad \mathbf{k} \in \widetilde{\Omega}, \quad|\mathbf{k}|>t_{0} .
$$

Hence,

$$
\left\|\widehat{\mathcal{A}}(\mathbf{k})^{1 / 2} f e^{-\mathcal{A}(\mathbf{k}) \tau} f^{*}\right\|_{L_{2}(\Omega) \rightarrow L_{2}(\Omega)} \leq \tau^{-1} c_{*}^{-1 / 2} t_{0}^{-1}\|f\|_{L_{\infty}}, \quad \tau>0, \quad \mathbf{k} \in \widetilde{\Omega}, \quad|\mathbf{k}|>t_{0} .
$$

Combining the identity $b(\mathbf{D}+\mathbf{k}) \widehat{P}=b(\mathbf{k}) \widehat{P}$ with (5.2), (8.3), (8.7), we obtain

$$
\begin{aligned}
& \left\|b(\mathbf{D}+\mathbf{k}) f_{0} e^{-\mathcal{A}^{0}(\mathbf{k}) \tau} f_{0} \widehat{P}\right\|_{L_{2}(\Omega) \rightarrow L_{2}(\Omega)} \leq \alpha_{1}^{1 / 2}\|f\|_{L_{\infty}}^{2}|\mathbf{k}| \exp \left(-c_{*}|\mathbf{k}|^{2} \tau\right) \\
& \quad \leq \tau^{-1} \alpha_{1}^{1 / 2}\|f\|_{L_{\infty}}^{2} c_{*}^{-1} t_{0}^{-1}, \quad \tau>0, \quad \mathbf{k} \in \widetilde{\Omega}, \quad|\mathbf{k}|>t_{0} .
\end{aligned}
$$

Hence,

$$
\begin{aligned}
& \left\|\widehat{\mathcal{A}}(\mathbf{k})^{1 / 2} f_{0} e^{-\mathcal{A}^{0}(\mathbf{k}) \tau} f_{0} \widehat{P}\right\|_{L_{2}(\Omega) \rightarrow L_{2}(\Omega)}=\left\|g^{1 / 2} b(\mathbf{D}+\mathbf{k}) f_{0} e^{-\mathcal{A}^{0}(\mathbf{k}) \tau} f_{0} \widehat{P}\right\|_{L_{2}(\Omega) \rightarrow L_{2}(\Omega)} \\
& \quad \leq \tau^{-1} \alpha_{1}^{1 / 2}\|g\|_{L_{\infty}}^{1 / 2}\|f\|_{L_{\infty}}^{2} c_{*}^{-1} t_{0}^{-1}, \quad \tau>0, \quad \mathbf{k} \in \widetilde{\Omega}, \quad|\mathbf{k}|>t_{0} .
\end{aligned}
$$

Next, from (8.24) and (7.18) it follows that

$$
\begin{array}{r}
\left\|\widehat{\mathcal{A}}(\mathbf{k})^{1 / 2} \Lambda b(\mathbf{D}+\mathbf{k}) f_{0} e^{-\mathcal{A}^{0}(\mathbf{k}) \tau} f_{0} \widehat{P}\right\|_{L_{2}(\Omega) \rightarrow L_{2}(\Omega)} \leq \tau^{-1} \alpha_{1}^{1 / 2}\|f\|_{L_{\infty}}^{2} c_{*}^{-1} t_{0}^{-1} \mathcal{C}_{\Lambda}, \\
\tau>0, \quad \mathbf{k} \in \widetilde{\Omega}, \quad|\mathbf{k}|>t_{0} .
\end{array}
$$

Finally, relations (8.23), (8.25), and (8.26) imply the estimate

$$
\begin{array}{r}
\left\|\widehat{\mathcal{A}}(\mathbf{k})^{1 / 2}\left(f e^{-\mathcal{A}(\mathbf{k}) \tau} f^{*}-(I+\Lambda b(\mathbf{D}+\mathbf{k})) f_{0} e^{-\mathcal{A}^{0}(\mathbf{k}) \tau} f_{0} \widehat{P}\right)\right\|_{L_{2}(\Omega) \rightarrow L_{2}(\Omega)} \leq C_{9} \tau^{-1} \\
\tau>0, \quad \mathbf{k} \in \widetilde{\Omega}, \quad|\mathbf{k}|>t_{0},
\end{array}
$$

where

$$
C_{9}=c_{*}^{-1 / 2} t_{0}^{-1}\|f\|_{L_{\infty}}+\alpha_{1}^{1 / 2}\|f\|_{L_{\infty}}^{2} c_{*}^{-1} t_{0}^{-1}\left(\|g\|_{L_{\infty}}^{1 / 2}+\mathcal{C}_{\Lambda}\right)
$$




\subsection{Further estimates. Final result}

Now, relations (8.21) and (8.27) show that for $\tau>0$ and $\mathbf{k} \in \widetilde{\Omega}$ we have

$$
\left\|\widehat{\mathcal{A}}(\mathbf{k})^{1 / 2}\left(f e^{-\mathcal{A}(\mathbf{k}) \tau} f^{*}-(I+\Lambda b(\mathbf{D}+\mathbf{k})) f_{0} e^{-\mathcal{A}^{0}(\mathbf{k}) \tau} f_{0} \widehat{P}\right)\right\|_{L_{2}(\Omega) \rightarrow L_{2}(\Omega)} \leq \widetilde{\mathcal{C}}_{5} \tau^{-1},
$$

where $\widetilde{\mathcal{C}}_{5}=\max \left\{C_{8}, C_{9}\right\}$.

Similarly to (7.22) and (7.23), taking (5.2), (8.3), and (8.6) into account, we obtain

$$
\begin{aligned}
& \left\|\widehat{\mathcal{A}}(\mathbf{k})^{1 / 2} f_{0} e^{-\mathcal{A}^{0}(\mathbf{k}) \tau} f_{0}(I-\widehat{P})\right\|_{L_{2}(\Omega) \rightarrow L_{2}(\Omega)} \leq \alpha_{1}^{1 / 2}\|g\|_{L_{\infty}}^{1 / 2}\|f\|_{L_{\infty}}^{2} \sup _{\substack{0 \neq \mathbf{b} \in \widetilde{\Gamma}\\
}}|\mathbf{b}+\mathbf{k}| e^{-c_{*}|\mathbf{b}+\mathbf{k}|^{2} \tau} \\
& \quad \leq \tau^{-1} \alpha_{1}^{1 / 2}\|g\|_{L_{\infty}}^{1 / 2}\|f\|_{L_{\infty}}^{2} c_{*}^{-1} r_{0}^{-1}, \quad \tau>0, \quad \mathbf{k} \in \widetilde{\Omega}
\end{aligned}
$$

From (8.28) and (8.29) it follows that

$\left\|\widehat{\mathcal{A}}(\mathbf{k})^{1 / 2}\left(f e^{-\mathcal{A}(\mathbf{k}) \tau} f^{*}-(I+\Lambda b(\mathbf{D}+\mathbf{k}) \widehat{P}) f_{0} e^{-\mathcal{A}^{0}(\mathbf{k}) \tau} f_{0}\right)\right\|_{L_{2}(\Omega) \rightarrow L_{2}(\Omega)} \leq \mathcal{C}_{5} \tau^{-1}, \tau>0, \mathbf{k} \in \widetilde{\Omega}$

where

$$
\mathcal{C}_{5}=\widetilde{\mathcal{C}}_{5}+\alpha_{1}^{1 / 2}\|g\|_{L_{\infty}}^{1 / 2}\|f\|_{L_{\infty}}^{2} c_{*}^{-1} r_{0}^{-1}
$$

If $0<\tau<1$, each term under the norm sign in (8.21) is estimated separately. By (8.22),

$$
\left\|\widehat{\mathcal{A}}(\mathbf{k})^{1 / 2} f e^{-\mathcal{A}(\mathbf{k}) \tau} f^{*}\right\|_{L_{2}(\Omega) \rightarrow L_{2}(\Omega)} \leq\|f\|_{L_{\infty}} \sup _{\lambda \geq 0} \lambda^{1 / 2} e^{-\lambda \tau} \leq \tau^{-1 / 2}\|f\|_{L_{\infty}}, \quad \tau>0, \quad \mathbf{k} \in \widetilde{\Omega} .
$$

Next, similarly to (8.29), we have

$$
\begin{aligned}
& \left\|b(\mathbf{D}+\mathbf{k}) f_{0} e^{-\mathcal{A}^{0}(\mathbf{k}) \tau} f_{0}\right\|_{L_{2}(\Omega) \rightarrow L_{2}(\Omega)} \leq \alpha_{1}^{1 / 2}\|f\|_{L_{\infty}}^{2} \sup _{\mathbf{b} \in \widetilde{\Gamma}}|\mathbf{b}+\mathbf{k}| \exp \left(-c_{*}|\mathbf{b}+\mathbf{k}|^{2} \tau\right) \\
& \quad \leq \tau^{-1 / 2} \alpha_{1}^{1 / 2}\|f\|_{L_{\infty}}^{2} c_{*}^{-1 / 2}, \quad \tau>0, \quad \mathbf{k} \in \widetilde{\Omega}
\end{aligned}
$$

Hence,

$$
\left\|\widehat{\mathcal{A}}(\mathbf{k})^{1 / 2} f_{0} e^{-\mathcal{A}^{0}(\mathbf{k}) \tau} f_{0}\right\|_{L_{2}(\Omega) \rightarrow L_{2}(\Omega)} \leq \tau^{-1 / 2} \alpha_{1}^{1 / 2}\|g\|_{L_{\infty}}^{1 / 2}\|f\|_{L_{\infty}}^{2} c_{*}^{-1 / 2}, \quad \tau>0, \quad \mathbf{k} \in \widetilde{\Omega} .
$$

From (7.18) and (8.33) it follows that

$$
\left\|\widehat{\mathcal{A}}(\mathbf{k})^{1 / 2} \Lambda b(\mathbf{D}+\mathbf{k}) f_{0} e^{-\mathcal{A}^{0}(\mathbf{k}) \tau} f_{0} \widehat{P}\right\|_{L_{2}(\Omega) \rightarrow L_{2}(\Omega)} \leq \tau^{-1 / 2} \alpha_{1}^{1 / 2}\|f\|_{L_{\infty}}^{2} c_{*}^{-1 / 2} \mathcal{C}_{\Lambda}, \quad \tau>0, \quad \mathbf{k} \in \widetilde{\Omega}
$$

Using (8.32), (8.34), and (8.35), we arrive at the estimate

$$
\begin{aligned}
\left\|\widehat{\mathcal{A}}(\mathbf{k})^{1 / 2}\left(f e^{-\mathcal{A}(\mathbf{k}) \tau} f^{*}-(I+\Lambda b(\mathbf{D}+\mathbf{k}) \widehat{P}) f_{0} e^{-\mathcal{A}^{0}(\mathbf{k}) \tau} f_{0}\right)\right\|_{L_{2}(\Omega) \rightarrow L_{2}(\Omega)} & \leq \mathcal{C}_{6} \tau^{-1 / 2} \\
\tau & >0, \quad \mathbf{k} \in \widetilde{\Omega}
\end{aligned}
$$


where

$$
\mathcal{C}_{6}=\|f\|_{L_{\infty}}+\alpha_{1}^{1 / 2}\|f\|_{L_{\infty}}^{2} c_{*}^{-1 / 2}\left(\|g\|_{L_{\infty}}^{1 / 2}+\mathcal{C}_{\Lambda}\right) .
$$

Estimate (8.30) is preferable for $\tau \geq 1$, and estimate (8.36) is preferable for $0<\tau<1$. We have proved the following result.

Theorem 8.1. Let $\widehat{\mathcal{A}}(\mathbf{k})$ be the operator family $(6.4)$, and let $\mathcal{A}(\mathbf{k})$ be the family (8.1). Let $\mathcal{A}^{0}(\mathbf{k})$ be the operator family (8.5). Let $\Lambda(\mathbf{x})$ be the $\Gamma$-periodic solution of problem (6.11). Let $\widehat{P}$ be the orthogonal projection (6.6). Then for $\tau>0$ and $\mathbf{k} \in \widetilde{\Omega}$ we have

$$
\begin{gathered}
\left\|\widehat{\mathcal{A}}(\mathbf{k})^{1 / 2}\left(f e^{-\mathcal{A}(\mathbf{k}) \tau} f^{*}-(I+\Lambda b(\mathbf{D}+\mathbf{k}) \widehat{P}) f_{0} e^{-\mathcal{A}^{0}(\mathbf{k}) \tau} f_{0}\right)\right\|_{L_{2}(\Omega) \rightarrow L_{2}(\Omega)} \leq \Phi_{2}(\tau) \\
\Phi_{2}(\tau)=\left\{\begin{array}{c}
\mathcal{C}_{5} \tau^{-1}, \quad \tau \geq 1 \\
\mathcal{C}_{6} \tau^{-1 / 2}, \quad 0<\tau<1 .
\end{array}\right.
\end{gathered}
$$

The constants $\mathcal{C}_{5}$ and $\mathcal{C}_{6}$ are defined by (8.31) and (8.37) respectively; they depend only on $m, \alpha_{0}, \alpha_{1},\|g\|_{L_{\infty}},\left\|g^{-1}\right\|_{L_{\infty}},\|f\|_{L_{\infty}},\left\|f^{-1}\right\|_{L_{\infty}}$, and the parameters of the lattice $\Gamma$.

\subsection{Approximation of the operator $g b(\mathbf{D}+\mathbf{k}) f e^{-\mathcal{A}(\mathbf{k}) \tau} f^{*}$}

Besides Theorem 8.1 we shall need the following statement.

Theorem 8.2. Suppose that the assumptions of Theorem 8.1 are satisfied. Let $\widetilde{g}(\mathbf{x})$ be the matrix (6.12). Then for $\tau>0$ and $\mathbf{k} \in \widetilde{\Omega}$ we have

$$
\begin{gathered}
\left\|g b(\mathbf{D}+\mathbf{k}) f e^{-\mathcal{A}(\mathbf{k}) \tau} f^{*}-\widetilde{g} b(\mathbf{D}+\mathbf{k}) f_{0} e^{-\mathcal{A}^{0}(\mathbf{k}) \tau} f_{0} \widehat{P}\right\|_{L_{2}(\Omega) \rightarrow L_{2}(\Omega)} \leq R_{2}(\tau), \\
R_{2}(\tau)=\left\{\begin{array}{c}
\mathcal{C}_{7} \tau^{-1}, \quad \tau \geq 1 \\
\mathcal{C}_{8} \tau^{-1 / 2}, \quad 0<\tau<1 .
\end{array}\right.
\end{gathered}
$$

The constants $\mathcal{C}_{7}, \mathcal{C}_{8}$ are defined below in (8.45); they depend only on $d, m, \alpha_{0}, \alpha_{1},\|g\|_{L_{\infty}}$, $\left\|g^{-1}\right\|_{L_{\infty}},\|f\|_{L_{\infty}},\left\|f^{-1}\right\|_{L_{\infty}}$, and the parameters of the lattice $\Gamma$.

Proof. For $\tau \geq 1$ we use estimate (8.28), and for $0<\tau<1$ we apply estimates (8.32), (8.34), (8.35). Then for $\tau>0$ and $\mathbf{k} \in \widetilde{\Omega}$ we obtain

$$
\begin{gathered}
\left\|g b(\mathbf{D}+\mathbf{k})\left(f e^{-\mathcal{A}(\mathbf{k}) \tau} f^{*}-(I+\Lambda b(\mathbf{D}+\mathbf{k})) f_{0} e^{-\mathcal{A}^{0}(\mathbf{k}) \tau} f_{0} \widehat{P}\right)\right\|_{L_{2}(\Omega) \rightarrow L_{2}(\Omega)} \leq \widetilde{R}_{2}(\tau), \\
\widetilde{R}_{2}(\tau)=\left\{\begin{array}{c}
\widetilde{\mathcal{C}}_{5}\|g\|_{L_{\infty}}^{1 / 2} \tau^{-1}, \quad \tau \geq 1, \\
\mathcal{C}_{6}\|g\|_{L_{\infty}}^{1 / 2} \tau^{-1 / 2}, \quad 0<\tau<1 .
\end{array}\right.
\end{gathered}
$$

Next, similarly to (7.35), we have

$$
\begin{aligned}
& g b(\mathbf{D}+\mathbf{k})(I+\Lambda b(\mathbf{D}+\mathbf{k})) f_{0} e^{-\mathcal{A}^{0}(\mathbf{k}) \tau} f_{0} \widehat{P}=\widetilde{g} b(\mathbf{D}+\mathbf{k}) f_{0} e^{-\mathcal{A}^{0}(\mathbf{k}) \tau} f_{0} \widehat{P} \\
& +g \sum_{j=1}^{d} b_{j} \Lambda\left(D_{j}+k_{j}\right) b(\mathbf{D}+\mathbf{k}) f_{0} e^{-\mathcal{A}^{0}(\mathbf{k}) \tau} f_{0} \widehat{P}
\end{aligned}
$$


Similarly to (7.38), taking (5.2), (8.3), and (8.7) into account, we obtain

$$
\left\|\left(D_{j}+k_{j}\right) b(\mathbf{D}+\mathbf{k}) f_{0} e^{-\mathcal{A}^{0}(\mathbf{k}) \tau} f_{0} \widehat{P}\right\|_{L_{2}(\Omega) \rightarrow L_{2}(\Omega)} \leq \alpha_{1}^{1 / 2}\|f\|_{L_{\infty}}^{2}|\mathbf{k}|^{2} \exp \left(-c_{*}|\mathbf{k}|^{2} \tau\right) .
$$

For $\tau \geq 1$ the term $|\mathbf{k}|^{2} \exp \left(-c_{*}|\mathbf{k}|^{2} \tau\right)$ is estimated by $c_{*}^{-1} \tau^{-1}$, and for $0<\tau<1$ it is estimated by $c_{*}^{-1 / 2} r_{1} \tau^{-1 / 2}$. Combining this with (5.3) and (7.37), we arrive at the estimate

$$
\begin{gathered}
\left\|g \sum_{j=1}^{d} b_{j} \Lambda\left(D_{j}+k_{j}\right) b(\mathbf{D}+\mathbf{k}) f_{0} e^{-\mathcal{A}^{0}(\mathbf{k}) \tau} f_{0} \widehat{P}\right\|_{L_{2}(\Omega) \rightarrow L_{2}(\Omega)} \\
\leq d m^{1 / 2}\left(2 r_{0}\right)^{-1} \alpha_{0}^{-1 / 2} \alpha_{1}\|g\|_{L_{\infty}}^{3 / 2}\left\|g^{-1}\right\|_{L_{\infty}}^{1 / 2}\|f\|_{L_{\infty}}^{2} \widetilde{\rho}(\tau), \\
\widetilde{\rho}(\tau)=\left\{\begin{array}{cc}
c_{*}^{-1} \tau^{-1}, & \tau \geq 1, \\
c_{*}^{-1 / 2} r_{1} \tau^{-1 / 2}, & 0<\tau<1 .
\end{array}\right.
\end{gathered}
$$

Relations (8.41), (8.42), and (8.44) imply (8.39) with

$$
\begin{aligned}
\mathcal{C}_{7} & =\widetilde{\mathcal{C}}_{5}\|g\|_{L_{\infty}}^{1 / 2}+d m^{1 / 2}\left(2 r_{0}\right)^{-1} \alpha_{0}^{-1 / 2} \alpha_{1}\|g\|_{L_{\infty}}^{3 / 2}\left\|g^{-1}\right\|_{L_{\infty}}^{1 / 2}\|f\|_{L_{\infty}}^{2} c_{*}^{-1} \\
\mathcal{C}_{8} & =\mathcal{C}_{6}\|g\|_{L_{\infty}}^{1 / 2}+d m^{1 / 2}\left(2 r_{0}\right)^{-1} \alpha_{0}^{-1 / 2} \alpha_{1}\|g\|_{L_{\infty}}^{3 / 2}\left\|g^{-1}\right\|_{L_{\infty}}^{1 / 2}\|f\|_{L_{\infty}}^{2} c_{*}^{-1 / 2} r_{1}
\end{aligned}
$$

\subsection{Special cases}

Relation (8.10) shows that under condition (7.41) we have $\Lambda_{Q}(\mathbf{x}) b(\boldsymbol{\theta})=0$ for $\mathbf{x} \in \Omega, \boldsymbol{\theta} \in \mathbb{S}^{d-1}$. Then (8.17) turns into the following inequality

$$
\left\|\widehat{\mathcal{A}}(\mathbf{k})^{1 / 2}\left(f e^{-\mathcal{A}(\mathbf{k}) \tau} f^{*}-f_{0} e^{-\mathcal{A}^{0}(\mathbf{k}) \tau} f_{0} \widehat{P}\right)\right\|_{L_{2}(\Omega) \rightarrow L_{2}(\Omega)} \leq C_{5}\|f\|_{L_{\infty}} \tau^{-1}, \quad \tau>0, \quad|\mathbf{k}| \leq t_{0} .
$$

For $\mathbf{k} \in \widetilde{\Omega}$ and $|\mathbf{k}|>t_{0}$ we use estimates (8.23) and (8.25). Hence,

$$
\left\|\widehat{\mathcal{A}}(\mathbf{k})^{1 / 2}\left(f e^{-\mathcal{A}(\mathbf{k}) \tau} f^{*}-f_{0} e^{-\mathcal{A}^{0}(\mathbf{k}) \tau} f_{0} \widehat{P}\right)\right\|_{L_{2}(\Omega) \rightarrow L_{2}(\Omega)} \leq \widetilde{\mathcal{C}}_{5}^{0} \tau^{-1}, \quad \tau>0, \quad \mathbf{k} \in \widetilde{\Omega}
$$

where

$$
\widetilde{\mathcal{C}}_{5}^{0}=\max \left\{C_{5}\|f\|_{L_{\infty}}, c_{*}^{-1 / 2} t_{0}^{-1}\|f\|_{L_{\infty}}+\alpha_{1}^{1 / 2}\|g\|_{L_{\infty}}^{1 / 2}\|f\|_{L_{\infty}}^{2} c_{*}^{-1} t_{0}^{-1}\right\} .
$$

We also take estimate (8.29) into account, and for $0<\tau<1$ apply inequalities (8.32) and (8.34). As a result, we obtain the following statement.

Proposition 8.3. Suppose that the assumptions of Theorem 8.1 and also condition (7.41) are satisfied. Then for $\tau>0$ and $\mathbf{k} \in \widetilde{\Omega}$ we have

$$
\begin{gathered}
\left\|\widehat{\mathcal{A}}(\mathbf{k})^{1 / 2}\left(f e^{-\mathcal{A}(\mathbf{k}) \tau} f^{*}-f_{0} e^{-\mathcal{A}^{0}(\mathbf{k}) \tau} f_{0}\right)\right\|_{L_{2}(\Omega) \rightarrow L_{2}(\Omega)} \leq \Phi_{2}^{0}(\tau) \\
\Phi_{2}^{0}(\tau)=\left\{\begin{array}{c}
\mathcal{C}_{5}^{0} \tau^{-1}, \quad \tau \geq 1 \\
\mathcal{C}_{6}^{0} \tau^{-1 / 2}, \quad 0<\tau<1 .
\end{array}\right.
\end{gathered}
$$


The constants $\mathcal{C}_{5}^{0}$ and $\mathcal{C}_{6}^{0}$ are defined by

$$
\mathcal{C}_{5}^{0}=\widetilde{\mathcal{C}}_{5}^{0}+\alpha_{1}^{1 / 2}\|g\|_{L_{\infty}}^{1 / 2}\|f\|_{L_{\infty}}^{2} c_{*}^{-1} r_{0}^{-1}, \quad \mathcal{C}_{6}^{0}=\|f\|_{L_{\infty}}+\alpha_{1}^{1 / 2}\|f\|_{L_{\infty}}^{2}\|g\|_{L_{\infty}}^{1 / 2} c_{*}^{-1 / 2}
$$

they depend only on $\alpha_{0}, \alpha_{1},\|g\|_{L_{\infty}},\left\|g^{-1}\right\|_{L_{\infty}},\|f\|_{L_{\infty}},\left\|f^{-1}\right\|_{L_{\infty}}$, and the parameters of the lattice $\Gamma$.

Finally, we consider a special case in approximation (8.39). Suppose that condition (6.20) is satisfied. Then $\widetilde{g}(\mathbf{x})=\underline{g}=g^{0}$, and for $\tau \geq 1$ relation (8.39) takes the form

$$
\left\|g b(\mathbf{D}+\mathbf{k}) f e^{-\mathcal{A}(\mathbf{k}) \tau} f^{*}-g^{0} b(\mathbf{D}+\mathbf{k}) f_{0} e^{-\mathcal{A}^{0}(\mathbf{k}) \tau} f_{0} \widehat{P}\right\|_{L_{2}(\Omega) \rightarrow L_{2}(\Omega)} \leq \mathcal{C}_{7} \tau^{-1}, \quad \tau \geq 1, \quad \mathbf{k} \in \widetilde{\Omega} .
$$

Similarly to (7.44), taking (5.2), (8.3), and (8.6) into account, we obtain

$$
\begin{aligned}
\left\|g^{0} b(\mathbf{D}+\mathbf{k}) f_{0} e^{-\mathcal{A}^{0}(\mathbf{k}) \tau} f_{0}(I-\widehat{P})\right\|_{L_{2}(\Omega) \rightarrow L_{2}(\Omega)} & \leq\left|g^{0}\right| \alpha_{1}^{1 / 2}\left|f_{0}\right|^{2} \sup _{0 \neq \mathbf{b} \in \widetilde{\Gamma}}|\mathbf{b}+\mathbf{k}| \exp \left(-c_{*}|\mathbf{b}+\mathbf{k}|^{2} \tau\right) \\
& \leq \tau^{-1} \alpha_{1}^{1 / 2}\|g\|_{L_{\infty}}\|f\|_{L_{\infty}}^{2} c_{*}^{-1} r_{0}^{-1}, \quad \tau>0, \quad \mathbf{k} \in \widetilde{\Omega}
\end{aligned}
$$

For $0<\tau<1$ we apply the estimate which follows from (8.32) and (8.33):

$$
\left\|g b(\mathbf{D}+\mathbf{k}) f e^{-\mathcal{A}(\mathbf{k}) \tau} f^{*}-g^{0} b(\mathbf{D}+\mathbf{k}) f_{0} e^{-\mathcal{A}^{0}(\mathbf{k}) \tau} f_{0}\right\|_{L_{2}(\Omega) \rightarrow L_{2}(\Omega)} \leq \mathcal{C}_{8}^{0} \tau^{-1 / 2}, \quad \tau>0, \quad \mathbf{k} \in \widetilde{\Omega},
$$

where

$$
\mathcal{C}_{8}^{0}=\|g\|_{L_{\infty}}^{1 / 2}\|f\|_{L_{\infty}}+\alpha_{1}^{1 / 2}\|g\|_{L_{\infty}}\|f\|_{L_{\infty}}^{2} c_{*}^{-1 / 2} .
$$

As a result, relations (8.47)-(8.49) imply the following statement.

Proposition 8.4. Suppose that the assumptions of Theorem 8.2 and also condition (6.20) are satisfied. Then for $\tau>0$ and $\mathbf{k} \in \widetilde{\Omega}$ we have

$$
\begin{gathered}
\left\|g b(\mathbf{D}+\mathbf{k}) f e^{-\mathcal{A}(\mathbf{k}) \tau} f^{*}-g^{0} b(\mathbf{D}+\mathbf{k}) f_{0} e^{-\mathcal{A}^{0}(\mathbf{k}) \tau} f_{0}\right\|_{L_{2}(\Omega) \rightarrow L_{2}(\Omega)} \leq R_{2}^{0}(\tau) \\
R_{2}^{0}(\tau)=\left\{\begin{array}{c}
\mathcal{C}_{7}^{0} \tau^{-1}, \quad \tau \geq 1 \\
\mathcal{C}_{8}^{0} \tau^{-1 / 2}, \quad 0<\tau<1 .
\end{array}\right.
\end{gathered}
$$

The constant $\mathcal{C}_{7}^{0}$ is given by $\mathcal{C}_{7}^{0}=\mathcal{C}_{7}+\alpha_{1}^{1 / 2}\|g\|_{L_{\infty}}\|f\|_{L_{\infty}}^{2} c_{*}^{-1} r_{0}^{-1}$, and $\mathcal{C}_{8}^{0}$ is defined by (8.50). The constant $\mathcal{C}_{8}^{0}$ depends only on $\alpha_{0}, \alpha_{1},\|g\|_{L_{\infty}},\left\|g^{-1}\right\|_{L_{\infty}},\|f\|_{L_{\infty}},\left\|f^{-1}\right\|_{L_{\infty}} ;$ the constant $\mathcal{C}_{7}^{0}$ depends on the same parameters and on $d, m$, and the parameters of the lattice $\Gamma$.

\section{Approximation of the operator $\exp (-\widehat{\mathcal{A}} \tau)$}

\subsection{Main theorem}

We return to the operator $\widehat{\mathcal{A}}=b(\mathbf{D})^{*} g(\mathbf{x}) b(\mathbf{D})$ acting in $L_{2}\left(\mathbb{R}^{d} ; \mathbb{C}^{n}\right)$. Let $g^{0}$ be the effective matrix, and let $\widehat{\mathcal{A}}^{0}=b(\mathbf{D})^{*} g^{0} b(\mathbf{D})$ be the effective operator. Let $\mathcal{V}$ denote the Gelfand 
transformation defined in Subsec. 5.3. Using expansion (5.20) for the operator $\widehat{\mathcal{A}}$, we represent the exponential $e^{-\widehat{\mathcal{A}} \tau}$ as

$$
e^{-\widehat{\mathcal{A}} \tau}=\mathcal{V}^{-1}\left(\int_{\widetilde{\Omega}} \oplus e^{-\widehat{\mathcal{A}}(\mathbf{k}) \tau} d \mathbf{k}\right) \mathcal{V}
$$

A similar expansion is valid also for $e^{-\widehat{\mathcal{A}}^{0} \tau}$. Under the Gelfand transformation, the operator $b(\mathbf{D})$ turns into $\int_{\widetilde{\Omega}} \oplus b(\mathbf{D}+\mathbf{k}) d \mathbf{k}$, and the operator of multiplication by the periodic matrixvalued function $\Lambda(\mathbf{x})$ turns into the operator of multiplication by the same function in the fibers of the direct integral $\mathcal{K}$ (see (5.11)). Also, we need the operator $\Pi=\mathcal{V}^{-1}[\widehat{P}] \mathcal{V}$ acting in $L_{2}\left(\mathbb{R}^{d} ; \mathbb{C}^{n}\right)$. Here $[\widehat{P}]$ is the projection in $\mathcal{K}$ that acts in the fibers as the operator $\widehat{P}$ (the operator of averaging over $\Omega$ ). As was shown in [BSu4, (6.8)], $\Pi$ is a pseudodifferential operator in $L_{2}\left(\mathbb{R}^{d} ; \mathbb{C}^{n}\right)$ with the symbol $\chi_{\widetilde{\Omega}}(\boldsymbol{\xi})$, where $\chi_{\widetilde{\Omega}}$ stands for the characteristic function of the set $\widetilde{\Omega}$. Thus,

$$
(\Pi \mathbf{u})(\mathbf{x})=(2 \pi)^{-d / 2} \int_{\widetilde{\Omega}} e^{i\langle\mathbf{x}, \boldsymbol{\xi}\rangle} \widehat{\mathbf{u}}(\boldsymbol{\xi}) d \boldsymbol{\xi}
$$

where $\widehat{\mathbf{u}}(\boldsymbol{\xi})$ is the Fourier image of $\mathbf{u}(\mathbf{x})$.

The next result follows directly from Theorem 7.1 by the Gelfand transformation.

Theorem 9.1. Let $\widehat{\mathcal{A}}=b(\mathbf{D})^{*} g(\mathbf{x}) b(\mathbf{D})$, and let $\widehat{\mathcal{A}}^{0}=b(\mathbf{D})^{*} g^{0} b(\mathbf{D})$ be the corresponding effective operator. Let $\Lambda(\mathbf{x})$ be the $\Gamma$-periodic solution of problem (6.11). Let $\Pi$ be the operator (9.1). Then for $\tau>0$ we have

$$
\left\|\widehat{\mathcal{A}}^{1 / 2}\left(e^{-\widehat{\mathcal{A}} \tau}-e^{-\widehat{\mathcal{A}}^{0} \tau}-\Lambda b(\mathbf{D}) e^{-\widehat{\mathcal{A}}^{0} \tau} \Pi\right)\right\|_{L_{2}\left(\mathbb{R}^{d}\right) \rightarrow L_{2}\left(\mathbb{R}^{d}\right)} \leq \Phi_{1}(\tau),
$$

where $\Phi_{1}(\tau)$ is defined by $(7.2)$.

\subsection{Elimination of the operator $\Pi$ in the corrector for $\tau \geq 1$}

Now we analyze the possibility to replace $\Pi$ by the identity operator in the corrector. We have to estimate the operator $\widehat{\mathcal{A}}^{1 / 2} \Lambda b(\mathbf{D}) e^{-\widehat{\mathcal{A}}^{0} \tau}(I-\Pi)$.

Proposition 9.2. For any $l>0$ the operator $b(\mathbf{D}) e^{-\widehat{\mathcal{A}}^{0} \tau}(I-\Pi)$ is a continuous mapping of $L_{2}\left(\mathbb{R}^{d} ; \mathbb{C}^{n}\right)$ to $H^{l}\left(\mathbb{R}^{d} ; \mathbb{C}^{m}\right)$, and

$$
\left\|b(\mathbf{D}) e^{-\widehat{\mathcal{A}}^{0} \tau}(I-\Pi)\right\|_{L_{2}\left(\mathbb{R}^{d}\right) \rightarrow H^{l}\left(\mathbb{R}^{d}\right)} \leq C_{l} \tau^{-(l+1) / 2}, \quad \tau>0 .
$$

The constant $C_{l}$ is defined below in (9.4) and depends only on $l, \alpha_{0}, \alpha_{1},\left\|g^{-1}\right\|_{L_{\infty}}$, and $r_{0}$.

Proof. The operator $b(\mathbf{D}) e^{-\widehat{\mathcal{A}}^{0} \tau}(I-\Pi)$ is a pseudodifferential operator with the symbol $b(\boldsymbol{\xi}) \exp \left(-b(\boldsymbol{\xi})^{*} g^{0} b(\boldsymbol{\xi}) \tau\right)\left(1-\chi_{\widetilde{\Omega}}(\boldsymbol{\xi})\right)$, whence

$$
\left\|b(\mathbf{D}) e^{-\widehat{\mathcal{A}}^{0} \tau}(I-\Pi)\right\|_{L_{2}\left(\mathbb{R}^{d}\right) \rightarrow H^{l}\left(\mathbb{R}^{d}\right)} \leq \sup _{\boldsymbol{\xi} \in \mathbb{R}^{d}}\left(1-\chi_{\widetilde{\Omega}}(\boldsymbol{\xi})\right)\left(1+|\boldsymbol{\xi}|^{2}\right)^{l / 2}\left|b(\boldsymbol{\xi}) \exp \left(-b(\boldsymbol{\xi})^{*} g^{0} b(\boldsymbol{\xi}) \tau\right)\right| .
$$


Combining this with (6.16), (5.2), and the estimate $|\boldsymbol{\xi}| \geq r_{0}$ for $\boldsymbol{\xi} \notin \widetilde{\Omega}$, we obtain

$$
\begin{aligned}
\left\|b(\mathbf{D}) e^{-\widehat{\mathcal{A}}^{0} \tau}(I-\Pi)\right\|_{L_{2}\left(\mathbb{R}^{d}\right) \rightarrow H^{l}\left(\mathbb{R}^{d}\right)} & \leq \alpha_{1}^{1 / 2} \sup _{|\boldsymbol{\xi}| \geq r_{0}}\left(1+|\boldsymbol{\xi}|^{2}\right)^{l / 2}|\boldsymbol{\xi}| \exp \left(-\widehat{c}_{*}|\boldsymbol{\xi}|^{2} \tau\right) \\
& \leq \tau^{-(l+1) / 2} c_{l} \alpha_{1}^{1 / 2}\left(\widehat{c}_{*}\right)^{-(l+1) / 2}\left(1+r_{0}^{-2}\right)^{l / 2}
\end{aligned}
$$

where

$$
c_{l}=\sup _{\alpha>0} \alpha^{(l+1) / 2} e^{-\alpha}=(l+1)^{(l+1) / 2}(2 e)^{-(l+1) / 2} .
$$

This proves estimate (9.3) with

$$
C_{l}=c_{l} \alpha_{1}^{1 / 2}\left(\widehat{c}_{*}\right)^{-(l+1) / 2}\left(1+r_{0}^{-2}\right)^{l / 2}
$$

Now we estimate the norm of $\widehat{\mathcal{A}}^{1 / 2}[\Lambda]$ as an operator acting from $H^{l}\left(\mathbb{R}^{d} ; \mathbb{C}^{m}\right.$ ) (with appropriate $l$ ) to $L_{2}\left(\mathbb{R}^{d} ; \mathbb{C}^{n}\right)$. Here $[\Lambda]$ denotes the operator of multiplication by the matrixvalued function $\Lambda(\mathbf{x})$. Let $\mathbf{v}_{j}, j=1, \ldots, m$, be the columns of the matrix $\Lambda(\mathbf{x})$. Then $\mathbf{v}_{j} \in \widetilde{H}^{1}\left(\Omega ; \mathbb{C}^{n}\right)$ is a weak $\Gamma$-periodic solution of the problem

$$
b(\mathbf{D})^{*} g(\mathbf{x})\left(b(\mathbf{D}) \mathbf{v}_{j}+\mathbf{e}_{j}\right)=0, \quad \int_{\Omega} \mathbf{v}_{j}(\mathbf{x}) d \mathbf{x}=0 .
$$

Here $\mathbf{e}_{1}, \ldots, \mathbf{e}_{m}$ is the standard orthonormal basis in $\mathbb{C}^{m}$. It is easy to check that (see [BSu4, Subsec. 6.3])

$$
\left\|\mathbf{v}_{j}\right\|_{H^{1}(\Omega)} \leq\left(1+1 / 4 r_{0}^{2}\right)^{1 / 2} \alpha_{0}^{-1 / 2}|\Omega|^{1 / 2}\|g\|_{L_{\infty}}^{1 / 2}\left\|g^{-1}\right\|_{L_{\infty}}^{1 / 2}, \quad j=1, \ldots, m .
$$

Proposition 9.3. Let $l=1$ for $d=1, l>1$ for $d=2$, and $l=d / 2$ for $d \geq 3$. The operator $\widehat{\mathcal{A}}^{1 / 2}[\Lambda]$ is a continuous mapping of $H^{l}\left(\mathbb{R}^{d} ; \mathbb{C}^{m}\right)$ to $L_{2}\left(\mathbb{R}^{d} ; \mathbb{C}^{n}\right)$, and

$$
\left\|\widehat{\mathcal{A}}^{1 / 2}[\Lambda]\right\|_{H^{l}\left(\mathbb{R}^{d}\right) \rightarrow L_{2}\left(\mathbb{R}^{d}\right)} \leq \mathfrak{C}_{d}
$$

where the constant $\mathfrak{C}_{d}$ depends only on $m, n, d, \alpha_{0}, \alpha_{1},\|g\|_{L_{\infty}},\left\|g^{-1}\right\|_{L_{\infty}}$, and the parameters of the lattice $\Gamma$; for $d=2$ it depends also on $l$.

Proof. It suffices to consider the operators $\widehat{\mathcal{A}}^{1 / 2}\left[\mathbf{v}_{j}\right]$ as operators acting from $H^{l}\left(\mathbb{R}^{d}\right)$ to $L_{2}\left(\mathbb{R}^{d} ; \mathbb{C}^{n}\right)$, for all $j=1, \ldots, m$. Let $u \in C_{0}^{\infty}\left(\mathbb{R}^{d}\right)$. According to (5.3), we have:

$$
b(\mathbf{D})\left(\mathbf{v}_{j} u\right)=\left(b(\mathbf{D}) \mathbf{v}_{j}\right) u+\sum_{k=1}^{d} b_{k} \mathbf{v}_{j}\left(D_{k} u\right) .
$$

Hence,

$$
\begin{aligned}
& \left\|\widehat{\mathcal{A}}^{1 / 2}\left(\mathbf{v}_{j} u\right)\right\|_{L_{2}\left(\mathbb{R}^{d}\right)}=\left\|g^{1 / 2} b(\mathbf{D})\left(\mathbf{v}_{j} u\right)\right\|_{L_{2}\left(\mathbb{R}^{d}\right)} \\
& \quad \leq\left\|g^{1 / 2}\left(b(\mathbf{D}) \mathbf{v}_{j}\right) u\right\|_{L_{2}\left(\mathbb{R}^{d}\right)}+\|g\|_{L_{\infty}}^{1 / 2} \alpha_{1}^{1 / 2} d^{1 / 2}\left(\int_{\mathbb{R}^{d}}\left|\mathbf{v}_{j}\right|^{2}|\mathbf{D} u|^{2} d \mathbf{x}\right)^{1 / 2} .
\end{aligned}
$$


Let us show that the first term in the right-hand side of (9.8) is controlled in terms of the second term and the norm of $u$ in $L_{2}\left(\mathbb{R}^{d}\right)$. Relation (9.5) implies that

$$
\int_{\mathbb{R}^{d}}\left\langle g(\mathbf{x})\left(b(\mathbf{D}) \mathbf{v}_{j}+\mathbf{e}_{j}\right), b(\mathbf{D}) \mathbf{w}\right\rangle d \mathbf{x}=0
$$

for any $\mathbf{w} \in H^{1}\left(\mathbb{R}^{d} ; \mathbb{C}^{n}\right)$ such that $\mathbf{w}(\mathbf{x})=0$ for $|\mathbf{x}|>R$ (with some $R>0$ ). As above, let $u \in C_{0}^{\infty}\left(\mathbb{R}^{d}\right)$. We put $\mathbf{w}=|u|^{2} \mathbf{v}_{j}$. Then

$$
b(\mathbf{D}) \mathbf{w}=|u|^{2}\left(b(\mathbf{D}) \mathbf{v}_{j}\right)+\sum_{k=1}^{d} b_{k}\left(D_{k}|u|^{2}\right) \mathbf{v}_{j} .
$$

Substituting this expression in (9.9), we obtain

$$
\int_{\mathbb{R}^{d}}\left\langle g(\mathbf{x})\left(b(\mathbf{D}) \mathbf{v}_{j}+\mathbf{e}_{j}\right),|u|^{2}\left(b(\mathbf{D}) \mathbf{v}_{j}\right)+\sum_{k=1}^{d} b_{k}\left(D_{k}|u|^{2}\right) \mathbf{v}_{j}\right\rangle d \mathbf{x}=0 .
$$

Consequently,

$$
\begin{aligned}
J & :=\int_{\mathbb{R}^{d}}\left|g^{1 / 2} b(\mathbf{D}) \mathbf{v}_{j}\right|^{2}|u|^{2} d \mathbf{x}=-\int_{\mathbb{R}^{d}}\left\langle g^{1 / 2} \mathbf{e}_{j}, g^{1 / 2} b(\mathbf{D}) \mathbf{v}_{j}\right\rangle|u|^{2} d \mathbf{x} \\
& -\int_{\mathbb{R}^{d}}\left\langle g^{1 / 2} b(\mathbf{D}) \mathbf{v}_{j}, g^{1 / 2} \sum_{k=1}^{d} b_{k}\left(D_{k}|u|^{2}\right) \mathbf{v}_{j}\right\rangle d \mathbf{x}-\int_{\mathbb{R}^{d}}\left\langle g \mathbf{e}_{j}, \sum_{k=1}^{d} b_{k}\left(D_{k}|u|^{2}\right) \mathbf{v}_{j}\right\rangle d \mathbf{x} .
\end{aligned}
$$

We estimate the first term on the right by $\frac{1}{4} J+\|g\|_{L_{\infty}}\|u\|_{L_{2}\left(\mathbb{R}^{d}\right)}^{2}$, the second one by

$$
\alpha_{1}^{1 / 2}\|g\|_{L_{\infty}}^{1 / 2} \int_{\mathbb{R}^{d}}\left|g^{1 / 2} b(\mathbf{D}) \mathbf{v}_{j}\right|\left(\sum_{k=1}^{d} 2\left|D_{k} u \| u\right|\right)\left|\mathbf{v}_{j}\right| d \mathbf{x} \leq \frac{1}{4} J+4 \alpha_{1}\|g\|_{L_{\infty}} d \int_{\mathbb{R}^{d}}\left|\mathbf{v}_{j}\right|^{2}|\mathbf{D} u|^{2} d \mathbf{x} .
$$

The third term is estimated by

$$
\alpha_{1}^{1 / 2}\|g\|_{L_{\infty}} \int_{\mathbb{R}^{d}} \sum_{k=1}^{d} 2\left|D_{k} u\left\|||\left|\mathbf{v}_{j}\right| d \mathbf{x} \leq\right\| g \|_{L_{\infty}} \int_{\mathbb{R}^{d}}\left(\alpha_{1}\left|\mathbf{v}_{j}\right|^{2}|\mathbf{D} u|^{2}+d|u|^{2}\right) d \mathbf{x} .\right.
$$

As a result, we obtain

$$
\frac{1}{2} \int_{\mathbb{R}^{d}}\left|g^{1 / 2} b(\mathbf{D}) \mathbf{v}_{j}\right|^{2}|u|^{2} d \mathbf{x} \leq\|g\|_{L_{\infty}}(d+1)\|u\|_{L_{2}\left(\mathbb{R}^{d}\right)}^{2}+\|g\|_{L_{\infty}} \alpha_{1}(4 d+1) \int_{\mathbb{R}^{d}}\left|\mathbf{v}_{j}\right|^{2}|\mathbf{D} u|^{2} d \mathbf{x}
$$

Relations (9.8) and (9.10) imply that

$$
\begin{aligned}
& \left\|\widehat{\mathcal{A}}^{1 / 2}\left(\mathbf{v}_{j} u\right)\right\|_{L_{2}\left(\mathbb{R}^{d}\right)} \leq\|g\|_{L_{\infty}}^{1 / 2}(2 d+2)^{1 / 2}\|u\|_{L_{2}\left(\mathbb{R}^{d}\right)} \\
& \quad+\|g\|_{L_{\infty}}^{1 / 2} \alpha_{1}^{1 / 2}\left((8 d+2)^{1 / 2}+d^{1 / 2}\right)\left(\int_{\mathbb{R}^{d}}\left|\mathbf{v}_{j}\right|^{2}|\mathbf{D} u|^{2} d \mathbf{x}\right)^{1 / 2} .
\end{aligned}
$$


So, the problem is reduced to estimating the last integral on the right.

It is convenient to assume that $1<l<2$ for $d=2$. (If the statement of Proposition is true for such $l$, then it is also true for larger l.) We use the embedding $H^{1}\left(\Omega ; \mathbb{C}^{n}\right) \subset L_{q}\left(\Omega ; \mathbb{C}^{n}\right)$, where $q=\infty$ for $d=1, q<\infty$ for $d=2$ and $q=2 d /(d-2)$ for $d \geq 3$. For $d=2$ we choose $q=2 /(l-1)$. Let $c(d, n)$ be the norm of the corresponding embedding operator. Then

$$
\left\|\mathbf{v}_{j}\right\|_{L_{q}(\Omega)} \leq c(d, n)\left\|\mathbf{v}_{j}\right\|_{H^{1}(\Omega)} .
$$

By the Hölder inequality,

$$
\int_{\Omega}\left|\mathbf{v}_{j}\right|^{2}|\mathbf{D} u|^{2} d \mathbf{x} \leq\left\|\mathbf{v}_{j}\right\|_{L_{q}(\Omega)}^{2}\|\mathbf{D} u\|_{L_{p}(\Omega)}^{2}
$$

where $p=2$ for $d=1, p=2 q /(q-2)=2 /(2-l)$ for $d=2, p=d$ for $d \geq 3$.

Now we use the embedding $H^{l-1}\left(\Omega ; \mathbb{C}^{d}\right) \subset L_{p}\left(\Omega ; \mathbb{C}^{d}\right)$, where $l=1$ and $p=2$ for $d=1$, $1<l<2$ and $p=2 /(2-l)$ for $d=2, l=d / 2$ and $p=d$ for $d \geq 3$. Let $\tilde{c}_{d}$ be the norm of the corresponding embedding operator. Then

$$
\|\mathbf{D} u\|_{L_{p}(\Omega)} \leq \tilde{c}_{d}\|u\|_{H^{l}(\Omega)} .
$$

As a result, relations (9.12)-(9.14) imply that

$$
\int_{\Omega}\left|\mathbf{v}_{j}\right|^{2}|\mathbf{D} u|^{2} d \mathbf{x} \leq c(d, n)^{2} \tilde{c}_{d}^{2}\left\|\mathbf{v}_{j}\right\|_{H^{1}(\Omega)}^{2}\|u\|_{H^{l}(\Omega)}^{2} .
$$

Since $\mathbf{v}_{j}$ is $\Gamma$-periodic, we can write inequality (9.15) for the shifted cells $\Omega+\mathbf{a}$ and sum over $\mathbf{a} \in \Gamma$. Then

$$
\int_{\mathbb{R}^{d}}\left|\mathbf{v}_{j}\right|^{2}|\mathbf{D} u|^{2} d \mathbf{x} \leq c(d, n)^{2} \tilde{c}_{d}^{2}\left\|\mathbf{v}_{j}\right\|_{H^{1}(\Omega)}^{2}\|u\|_{H^{l}\left(\mathbb{R}^{d}\right)}^{2} .
$$

Finally, combining (9.11), (9.16), and (9.6), we arrive at the estimate

$$
\left\|\widehat{\mathcal{A}}^{1 / 2}\left(\mathbf{v}_{j} u\right)\right\|_{L_{2}\left(\mathbb{R}^{d}\right)} \leq \check{\mathfrak{C}}_{d}\|u\|_{H^{l}\left(\mathbb{R}^{d}\right)},
$$

where

$\check{\mathfrak{C}}_{d}=\|g\|_{L_{\infty}}^{1 / 2}(2 d+2)^{1 / 2}+\left((8 d+2)^{1 / 2}+d^{1 / 2}\right) c(d, n) \tilde{c}_{d}\left(1+1 / 4 r_{0}^{2}\right)^{1 / 2} \alpha_{0}^{-1 / 2} \alpha_{1}^{1 / 2}|\Omega|^{1 / 2}\|g\|_{L_{\infty}}\left\|g^{-1}\right\|_{L_{\infty}}^{1 / 2}$.

This implies (9.7) with the constant $\mathfrak{C}_{d}=\check{\mathfrak{C}}_{d} m^{1 / 2}$.

From Propositions 9.2 and 9.3 it follows that

$$
\left\|\widehat{\mathcal{A}}^{1 / 2} \Lambda b(\mathbf{D}) e^{-\widehat{\mathcal{A}}^{0} \tau}(I-\Pi)\right\|_{L_{2}\left(\mathbb{R}^{d}\right) \rightarrow L_{2}\left(\mathbb{R}^{d}\right)} \leq C_{l} \mathfrak{C}_{d} \tau^{-(l+1) / 2}, \quad \tau>0,
$$

where $l=1$ for $d=1, l>1$ for $d=2$, and $l=d / 2$ for $d \geq 3$. If $\tau \geq 1$, then $\tau^{-(l+1) / 2} \leq \tau^{-1}$. Let us fix $l$ in the case $d=2$ (for instance, $l=3 / 2$ ). Together with the statement of Theorem 9.1 for the case $\tau \geq 1$ the above arguments imply the following result.

Theorem 9.4. Suppose that the assumptions of Theorem 9.1 are satisfied. Then we have

$$
\left\|\widehat{\mathcal{A}}^{1 / 2}\left(e^{-\widehat{\mathcal{A}} \tau}-(I+\Lambda b(\mathbf{D})) e^{-\widehat{\mathcal{A}}^{0} \tau}\right)\right\|_{L_{2}\left(\mathbb{R}^{d}\right) \rightarrow L_{2}\left(\mathbb{R}^{d}\right)} \leq \mathcal{C}_{1}^{\prime} \tau^{-1}, \quad \tau \geq 1 .
$$

The constant $\mathcal{C}_{1}^{\prime}=\mathcal{C}_{1}+C_{l} \mathfrak{C}_{d}$ depends only on $d, m, n, \alpha_{0}, \alpha_{1},\|g\|_{L_{\infty}},\left\|g^{-1}\right\|_{L_{\infty}}$, and the parameters of the lattice $\Gamma$. 


\subsection{Approximation of the operator $g b(\mathbf{D}) \exp (-\widehat{\mathcal{A}} \tau)$}

By the Gelfand transformation, Theorem 7.2 implies the following result.

Theorem 9.5. Suppose that the assumptions of Theorem 9.1 are satisfied. Let $\widetilde{g}(\mathbf{x})$ be the matrix (6.12). Then for $\tau>0$ we have

$$
\left\|g b(\mathbf{D}) e^{-\widehat{\mathcal{A}} \tau}-\widetilde{g} b(\mathbf{D}) e^{-\widehat{\mathcal{A}}^{0} \tau} \Pi\right\|_{L_{2}\left(\mathbb{R}^{d}\right) \rightarrow L_{2}\left(\mathbb{R}^{d}\right)} \leq R_{1}(\tau),
$$

where $R_{1}(\tau)$ is defined by (7.31).

We show that for $\tau \geq 1$ it is possible to replace the operator $\Pi$ by $I$ in (9.18). Only the constant in estimate will change under such replacement.

Proposition 9.6. Let $l=1$ for $d=1, l>1$ for $d=2$, and $l=d / 2$ for $d \geq 3$. The operator $[\widetilde{g}]$ of multiplication by the matrix-valued function $\widetilde{g}(\mathbf{x})$ is a continuos mapping of $H^{l}\left(\mathbb{R}^{d} ; \mathbb{C}^{m}\right)$ to $L_{2}\left(\mathbb{R}^{d} ; \mathbb{C}^{m}\right)$, and

$$
\|[\widetilde{g}]\|_{H^{l}\left(\mathbb{R}^{d}\right) \rightarrow L_{2}\left(\mathbb{R}^{d}\right)} \leq \mathfrak{C}_{d}^{\prime}
$$

The constant $\mathfrak{C}_{d}^{\prime}$ is defined below in (9.21) and depends only on $d, m, n, \alpha_{0}, \alpha_{1},\|g\|_{L_{\infty}}$, $\left\|g^{-1}\right\|_{L_{\infty}}$, and the parameters of the lattice $\Gamma$; for $d=2$ it depends also on $l$.

Proof. Let $\mathbf{v}_{j}(\mathbf{x}), j=1, \ldots, m$, be the columns of the matrix $\Lambda(\mathbf{x})$. From (9.10), (9.16), and (9.6) it follows that

$$
\begin{gathered}
\left\|\left[g^{1 / 2} b(\mathbf{D}) \mathbf{v}_{j}\right]\right\|_{H^{l}\left(\mathbb{R}^{d}\right) \rightarrow L_{2}\left(\mathbb{R}^{d}\right)} \leq \mathfrak{C}_{d}^{\prime \prime}, \quad j=1, \ldots, m, \\
\mathfrak{C}_{d}^{\prime \prime}=\|g\|_{L_{\infty}}^{1 / 2}(2 d+2)^{1 / 2}+(8 d+2)^{1 / 2} c(d, n) \tilde{c}_{d}\left(1+1 / 4 r_{0}^{2}\right)^{1 / 2} \alpha_{0}^{-1 / 2} \alpha_{1}^{1 / 2}|\Omega|^{1 / 2}\|g\|_{L_{\infty}}\left\|g^{-1}\right\|_{L_{\infty}}^{1 / 2} .
\end{gathered}
$$

Hence,

$$
\|[g b(\mathbf{D}) \Lambda]\|_{H^{l}\left(\mathbb{R}^{d}\right) \rightarrow L_{2}\left(\mathbb{R}^{d}\right)} \leq m^{1 / 2}\|g\|_{L_{\infty}}^{1 / 2} \mathfrak{C}_{d}^{\prime \prime} .
$$

Since $\widetilde{g}=g+g b(\mathbf{D}) \Lambda$, then (9.20) implies (9.19) with

$$
\mathfrak{C}_{d}^{\prime}=\|g\|_{L_{\infty}}+m^{1 / 2}\|g\|_{L_{\infty}}^{1 / 2} \mathfrak{C}_{d}^{\prime \prime}
$$

From Propositions 9.2 and 9.6 it follows that

$$
\left\|\widetilde{g} b(\mathbf{D}) e^{-\widehat{\mathcal{A}}^{0} \tau}(I-\Pi)\right\|_{L_{2}\left(\mathbb{R}^{d}\right) \rightarrow L_{2}\left(\mathbb{R}^{d}\right)} \leq C_{l} \mathfrak{C}_{d}^{\prime} \tau^{-(l+1) / 2}, \quad \tau>0,
$$

where $l$ is such as in Proposition 9.6. In the case $d=2$ we fix $l$ (for instance, $l=3 / 2$ ). Together with the statement of Theorem 9.5 for the case $\tau \geq 1$ this leads to the following result.

Theorem 9.7. Suppose that the assumptions of Theorem 9.5 are satisfied. Then

$$
\left\|g b(\mathbf{D}) e^{-\widehat{\mathcal{A}} \tau}-\widetilde{g} b(\mathbf{D}) e^{-\widehat{\mathcal{A}}^{0} \tau}\right\|_{L_{2}\left(\mathbb{R}^{d}\right) \rightarrow L_{2}\left(\mathbb{R}^{d}\right)} \leq \mathcal{C}_{3}^{\prime} \tau^{-1}, \quad \tau \geq 1 .
$$

The constant $\mathcal{C}_{3}^{\prime}=\mathcal{C}_{3}+C_{l} \mathfrak{C}_{d}^{\prime \prime}$ depends only on $d, m, n, \alpha_{0}, \alpha_{1},\|g\|_{L_{\infty}},\left\|g^{-1}\right\|_{L_{\infty}}$, and the parameters of the lattice $\Gamma$. 


\subsection{Special cases}

By the Gelfand transformation, Proposition 7.3 implies the following statement.

Proposition 9.8. Suppose that the assumptions of Theorem 9.1 and also condition (7.41) are satisfied. Then we have

$$
\left\|\widehat{\mathcal{A}}^{1 / 2}\left(e^{-\widehat{\mathcal{A}} \tau}-e^{-\widehat{\mathcal{A}}^{0} \tau}\right)\right\|_{L_{2}\left(\mathbb{R}^{d}\right) \rightarrow L_{2}\left(\mathbb{R}^{d}\right)} \leq \Phi_{1}^{0}(\tau), \quad \tau>0,
$$

where $\Phi_{1}^{0}(\tau)$ is defined by (7.42).

Similarly, Proposition 7.4 implies the following result.

Proposition 9.9. Suppose that the assumptions of Theorem 9.7 and also condition (6.20) are satisfied. Then we have

$$
\left\|g b(\mathbf{D}) e^{-\widehat{\mathcal{A}} \tau}-g^{0} b(\mathbf{D}) e^{-\widehat{\mathcal{A}}^{0} \tau}\right\|_{L_{2}\left(\mathbb{R}^{d}\right) \rightarrow L_{2}\left(\mathbb{R}^{d}\right)} \leq R_{1}^{0}(\tau), \quad \tau>0,
$$

where $R_{1}^{0}(\tau)$ is defined by $(7.47)$.

\section{Approximation of the bordered exponential $e^{-\mathcal{A} \tau}$}

\subsection{Approximation of the operator $f e^{-\mathcal{A} \tau} f^{*}$}

By the Gelfand transformation, Theorem 8.1 implies the following result.

Theorem 10.1. Let $\widehat{\mathcal{A}}=b(\mathbf{D})^{*} g(\mathbf{x}) b(\mathbf{D})$, and let $\mathcal{A}=f^{*} \widehat{\mathcal{A}} f$. Let $\mathcal{A}^{0}=f_{0} b(\mathbf{D})^{*} g^{0} b(\mathbf{D}) f_{0}$, where $f_{0}$ is defined by (8.2), and $g^{0}$ is the effective matrix (6.13). Let $\Lambda(\mathbf{x})$ be the $\Gamma$-periodic solution of problem (6.11). Let $\Pi$ be the operator (9.1). Then for $\tau>0$ we have

$$
\left\|\widehat{\mathcal{A}}^{1 / 2}\left(f e^{-\mathcal{A} \tau} f^{*}-f_{0} e^{-\mathcal{A}^{0} \tau} f_{0}-\Lambda b(\mathbf{D}) f_{0} e^{-\mathcal{A}^{0} \tau} f_{0} \Pi\right)\right\|_{L_{2}\left(\mathbb{R}^{d}\right) \rightarrow L_{2}\left(\mathbb{R}^{d}\right)} \leq \Phi_{2}(\tau),
$$

where $\Phi_{2}(\tau)$ is defined by (8.38).

It is easy to prove the following statement by analogy with Proposition 9.2.

Proposition 10.2. For any $l>0$ the operator $b(\mathbf{D}) f_{0} e^{-\mathcal{A}^{0} \tau} f_{0}(I-\Pi)$ is a continuous mapping of $L_{2}\left(\mathbb{R}^{d} ; \mathbb{C}^{n}\right)$ to $H^{l}\left(\mathbb{R}^{d} ; \mathbb{C}^{m}\right)$, and

$$
\left\|b(\mathbf{D}) f_{0} e^{-\mathcal{A}^{0} \tau} f_{0}(I-\Pi)\right\|_{L_{2} \rightarrow H^{l}} \leq \widetilde{C}_{l} \tau^{-(l+1) / 2}, \quad \tau>0,
$$

where the constant $\widetilde{C}_{l}$ is given by $\widetilde{C}_{l}=c_{l} \alpha_{1}^{1 / 2} c_{*}^{-(l+1) / 2}\|f\|_{L_{\infty}}^{2}\left(1+r_{0}^{-2}\right)^{l / 2}$ and depends only on $l, \alpha_{0}, \alpha_{1},\left\|g^{-1}\right\|_{L_{\infty}},\|f\|_{L_{\infty}},\left\|f^{-1}\right\|_{L_{\infty}}$, and $r_{0}$.

Now Propositions 9.3 and 10.2 imply the estimate

$$
\left\|\widehat{\mathcal{A}}^{1 / 2} \Lambda b(\mathbf{D}) f_{0} e^{-\mathcal{A}^{0} \tau} f_{0}(I-\Pi)\right\|_{L_{2}\left(\mathbb{R}^{d}\right) \rightarrow L_{2}\left(\mathbb{R}^{d}\right)} \leq \widetilde{C}_{l} \mathfrak{C}_{d} \tau^{-(l+1) / 2}, \quad \tau>0
$$


where $l$ is such as in Proposition 9.3. For $d=2$ we fix $l$ (for instance, $l=3 / 2$ ). Together with the statement of Theorem 10.1 for the case $\tau \geq 1$ this implies the following result.

Theorem 10.3. Suppose that the assumptions of Theorem 10.1 are satisfied. Then we have

$$
\left\|\widehat{\mathcal{A}}^{1 / 2}\left(f e^{-\mathcal{A} \tau} f^{*}-(I+\Lambda b(\mathbf{D})) f_{0} e^{-\mathcal{A}^{0} \tau} f_{0}\right)\right\|_{L_{2}\left(\mathbb{R}^{d}\right) \rightarrow L_{2}\left(\mathbb{R}^{d}\right)} \leq \mathcal{C}_{5}^{\prime} \tau^{-1}, \quad \tau \geq 1
$$

The constant $\mathcal{C}_{5}^{\prime}=\mathcal{C}_{5}+\mathfrak{C}_{d} \widetilde{C}_{l}$ depends only on $d, m, n, \alpha_{0}, \alpha_{1},\|g\|_{L_{\infty}},\left\|g^{-1}\right\|_{L_{\infty}},\|f\|_{L_{\infty}}$, $\left\|f^{-1}\right\|_{L_{\infty}}$, and the parameters of the lattice $\Gamma$.

\subsection{Approximation of the operator $g b(\mathbf{D}) f e^{-\mathcal{A} \tau} f^{*}$}

By the Gelfand transformation, Theorem 8.2 implies the following result.

Theorem 10.4. Suppose that the assumptions of Theorem 10.1 are satisfied. Let $\widetilde{g}(\mathbf{x})$ be the matrix (6.12). Then for $\tau>0$ we have

$$
\left\|g b(\mathbf{D}) f e^{-\mathcal{A} \tau} f^{*}-\tilde{g} b(\mathbf{D}) f_{0} e^{-\mathcal{A}^{0} \tau} f_{0} \Pi\right\|_{L_{2}\left(\mathbb{R}^{d}\right) \rightarrow L_{2}\left(\mathbb{R}^{d}\right)} \leq R_{2}(\tau),
$$

where $R_{2}(\tau)$ is defined by $(8.40)$.

Propositions 10.2 and 9.6 imply that

$$
\left\|\widetilde{g} b(\mathbf{D}) f_{0} e^{-\mathcal{A}^{0} \tau} f_{0}(I-\Pi)\right\|_{L_{2}\left(\mathbb{R}^{d}\right) \rightarrow L_{2}\left(\mathbb{R}^{d}\right)} \leq \widetilde{C}_{l} \mathfrak{C}_{d}^{\prime} \tau^{-(l+1) / 2}, \quad \tau>0,
$$

where $l$ is such as in Proposition 9.6. For $d=2$ we fix $l$ (for instance, $l=3 / 2$ ). Combining this with the statement of Theorem 10.4 for the case $\tau \geq 1$, we arrive at the following result.

Theorem 10.5. Suppose that the assumptions of Theorem 10.4 are satisfied. Then we have

$$
\left\|g b(\mathbf{D}) f e^{-\mathcal{A} \tau} f^{*}-\widetilde{g} b(\mathbf{D}) f_{0} e^{-\mathcal{A}^{0} \tau} f_{0}\right\|_{L_{2}\left(\mathbb{R}^{d}\right) \rightarrow L_{2}\left(\mathbb{R}^{d}\right)} \leq \mathcal{C}_{7}^{\prime} \tau^{-1}, \quad \tau \geq 1
$$

where the constant $\mathcal{C}_{7}^{\prime}=\mathcal{C}_{7}+\widetilde{C}_{l} \mathfrak{C}_{d}^{\prime}$ depends only on $d, m, n, \alpha_{0}, \alpha_{1},\|g\|_{L_{\infty}},\left\|g^{-1}\right\|_{L_{\infty}},\|f\|_{L_{\infty}}$, $\left\|f^{-1}\right\|_{L_{\infty}}$, and the parameters of the lattice $\Gamma$.

\subsection{Special cases}

By the Gelfand transformation, the following statement is deduced from Proposition 8.3.

Proposition 10.6. Suppose that the assumptions of Theorem 10.1 and also condition (7.41) are satisfied. Then we have

$$
\left\|\widehat{\mathcal{A}}^{1 / 2}\left(f e^{-\mathcal{A} \tau} f^{*}-f_{0} e^{-\mathcal{A}^{0} \tau} f_{0}\right)\right\|_{L_{2}\left(\mathbb{R}^{d}\right) \rightarrow L_{2}\left(\mathbb{R}^{d}\right)} \leq \Phi_{2}^{0}(\tau), \quad \tau>0
$$


where $\Phi_{2}^{0}(\tau)$ is defined by (8.46).

Similarly, Proposition 8.4 implies the following statement.

Proposition 10.7. Suppose that the assumptions of Theorem 10.1 and also condition (6.20) are satisfied. Then we have

$$
\left\|g b(\mathbf{D}) f e^{-\mathcal{A} \tau} f^{*}-g^{0} b(\mathbf{D}) f_{0} e^{-\mathcal{A}^{0} \tau} f_{0}\right\|_{L_{2}\left(\mathbb{R}^{d}\right) \rightarrow L_{2}\left(\mathbb{R}^{d}\right)} \leq R_{2}^{0}(\tau), \quad \tau>0,
$$

where $R_{2}^{0}(\tau)$ is defined by (8.51).

\section{Chapter 3. Homogenization of periodic DO's}

We proceed to the homogenization problems in the small period limit for periodic differential operators acting in $L_{2}\left(\mathbb{R}^{d} ; \mathbb{C}^{n}\right)$. If $\phi(\mathbf{x})$ is a measurable $\Gamma$-periodic function in $\mathbb{R}^{d}$, we denote $\phi^{\varepsilon}(\mathbf{x}):=\phi\left(\varepsilon^{-1} \mathbf{x}\right), \varepsilon>0$. We consider the operators

$$
\widehat{\mathcal{A}}_{\varepsilon}=b(\mathbf{D})^{*} g^{\varepsilon}(\mathbf{x}) b(\mathbf{D}), \quad \mathcal{A}_{\varepsilon}=\left(f^{\varepsilon}(\mathbf{x})\right)^{*} b(\mathbf{D})^{*} g^{\varepsilon}(\mathbf{x}) b(\mathbf{D}) f^{\varepsilon}(\mathbf{x}),
$$

with coefficients oscillating rapidly as $\varepsilon \rightarrow 0$. Our goal is to obtain approximation for the operators $\exp \left(-\widehat{\mathcal{A}}_{\varepsilon} \tau\right)$ and $f^{\varepsilon} \exp \left(-\mathcal{A}_{\varepsilon} \tau\right)\left(f^{\varepsilon}\right)^{*}$ for a fixed $\tau>0$ in the norm of operators acting from $L_{2}\left(\mathbb{R}^{d} ; \mathbb{C}^{n}\right)$ to $H^{1}\left(\mathbb{R}^{d} ; \mathbb{C}^{n}\right)$.

\section{Approximation of the operator $\exp \left(-\widehat{\mathcal{A}}_{\varepsilon} \tau\right)$}

\subsection{Main theorem}

Consider the scaling transformation $T_{\varepsilon}$ given by $\left(T_{\varepsilon} \mathbf{u}\right)(\mathbf{y})=\varepsilon^{d / 2} \mathbf{u}(\varepsilon \mathbf{y}), \mathbf{y} \in \mathbb{R}^{d}$. The operator $T_{\varepsilon}$ is unitary in $L_{2}\left(\mathbb{R}^{d} ; \mathbb{C}^{n}\right)$. The following identities are true:

$$
\begin{array}{r}
\widehat{\mathcal{A}}_{\varepsilon}=\varepsilon^{-2} T_{\varepsilon}^{*} \widehat{\mathcal{A}} T_{\varepsilon}, \quad \widehat{\mathcal{A}}_{\varepsilon}^{1 / 2}=\varepsilon^{-1} T_{\varepsilon}^{*} \widehat{\mathcal{A}}^{1 / 2} T_{\varepsilon}, \\
\exp \left(-\widehat{\mathcal{A}}_{\varepsilon} \tau\right)=T_{\varepsilon}^{*} \exp \left(-\widehat{\mathcal{A}} \tau \varepsilon^{-2}\right) T_{\varepsilon}, \\
\exp \left(-\widehat{\mathcal{A}}^{0} \tau\right)=T_{\varepsilon}^{*} \exp \left(-\widehat{\mathcal{A}}^{0} \tau \varepsilon^{-2}\right) T_{\varepsilon}, \\
b(\mathbf{D})=\varepsilon^{-1} T_{\varepsilon}^{*} b(\mathbf{D}) T_{\varepsilon}, \quad\left[\Lambda^{\varepsilon}\right]=T_{\varepsilon}^{*}[\Lambda] T_{\varepsilon} .
\end{array}
$$

We put $\Pi_{\varepsilon}:=T_{\varepsilon}^{*} \Pi T_{\varepsilon}$. Then $\Pi_{\varepsilon}$ is a pseudodifferential operator in $L_{2}\left(\mathbb{R}^{d} ; \mathbb{C}^{n}\right)$ with the symbol $\chi_{\widetilde{\Omega} / \varepsilon}(\boldsymbol{\xi})$, i. e.,

$$
\left(\Pi_{\varepsilon} \mathbf{u}\right)(\mathbf{x})=(2 \pi)^{-d / 2} \int_{\widetilde{\Omega} / \varepsilon} e^{i\langle\mathbf{x}, \boldsymbol{\xi}\rangle} \widehat{\mathbf{u}}(\boldsymbol{\xi}) d \boldsymbol{\xi}
$$

Thus, we have

$$
\begin{aligned}
& \widehat{\mathcal{A}}_{\varepsilon}^{1 / 2}\left(e^{-\widehat{\mathcal{A}}_{\varepsilon} \tau}-\left(I+\varepsilon \Lambda^{\varepsilon} b(\mathbf{D}) \Pi_{\varepsilon}\right) e^{-\widehat{\mathcal{A}}^{0} \tau}\right) \\
& =\varepsilon^{-1} T_{\varepsilon}^{*} \widehat{\mathcal{A}}^{1 / 2}\left(e^{-\widehat{\mathcal{A}} \tau \varepsilon^{-2}}-(I+\Lambda b(\mathbf{D}) \Pi) e^{-\widehat{\mathcal{A}}^{0} \tau \varepsilon^{-2}}\right) T_{\varepsilon} .
\end{aligned}
$$


Applying inequality (9.2) with $\tau$ replaced by $\tau \varepsilon^{-2}$ and identity (11.2) and taking into account that $T_{\varepsilon}$ is unitary operator, we obtain

$$
\begin{gathered}
\left\|\widehat{\mathcal{A}}_{\varepsilon}^{1 / 2}\left(e^{-\widehat{\mathcal{A}}_{\varepsilon} \tau}-\left(I+\varepsilon \Lambda^{\varepsilon} b(\mathbf{D}) \Pi_{\varepsilon}\right) e^{-\widehat{\mathcal{A}}^{0} \tau}\right)\right\|_{L_{2}\left(\mathbb{R}^{d}\right) \rightarrow L_{2}\left(\mathbb{R}^{d}\right)} \leq \widetilde{\Psi}_{1}(\tau, \varepsilon), \quad \tau>0, \quad \varepsilon>0, \\
\widetilde{\Psi}_{1}(\tau, \varepsilon)=\left\{\begin{array}{cc}
\mathcal{C}_{1} \tau^{-1} \varepsilon, & \tau>0, \quad 0<\varepsilon \leq \tau^{1 / 2} \\
\mathcal{C}_{2} \tau^{-1 / 2}, & \tau>0, \quad \varepsilon>\tau^{1 / 2}
\end{array}\right.
\end{gathered}
$$

Using (11.3), we shall obtain approximation of the operator $e^{-\widehat{\mathcal{A}}_{\varepsilon} \tau}$ in the $\left(L_{2} \rightarrow H^{1}\right)$ norm. Similarly to (5.5), we have

$$
\widehat{c}_{*} \int_{\mathbb{R}^{d}}|\mathbf{D u}|^{2} d \mathbf{x} \leq\left\|\widehat{\mathcal{A}}_{\varepsilon}^{1 / 2} \mathbf{u}\right\|_{L_{2}\left(\mathbb{R}^{d}\right)}^{2}, \quad \mathbf{u} \in H^{1}\left(\mathbb{R}^{d} ; \mathbb{C}^{n}\right) .
$$

Combining this with (11.3), we arrive at the estimate

$$
\left\|\mathbf{D}\left(e^{-\widehat{\mathcal{A}}_{\varepsilon} \tau}-\left(I+\varepsilon \Lambda^{\varepsilon} b(\mathbf{D}) \Pi_{\varepsilon}\right) e^{-\widehat{\mathcal{A}}^{0} \tau}\right)\right\|_{L_{2}\left(\mathbb{R}^{d}\right) \rightarrow L_{2}\left(\mathbb{R}^{d}\right)} \leq\left(\widehat{c}_{*}\right)^{-1 / 2} \widetilde{\Psi}_{1}(\tau, \varepsilon) .
$$

It remains to estimate the operator $e^{-\widehat{\mathcal{A}}_{\varepsilon} \tau}-\left(I+\varepsilon \Lambda^{\varepsilon} b(\mathbf{D}) \Pi_{\varepsilon}\right) e^{-\widehat{\mathcal{A}}^{0} \tau}$ in the $\left(L_{2} \rightarrow L_{2}\right)$-norm. According to [Su2, Theorem 7.1], we have

$$
\left\|e^{-\widehat{\mathcal{A}}_{\varepsilon} \tau}-e^{-\widehat{\mathcal{A}}^{0} \tau}\right\|_{L_{2}\left(\mathbb{R}^{d}\right) \rightarrow L_{2}\left(\mathbb{R}^{d}\right)} \leq \widehat{\mathcal{C}}_{0} \tau^{-1 / 2} \varepsilon, \quad \tau>0, \quad \varepsilon>0
$$

The constant $\widehat{\mathcal{C}_{0}}$ depends only on $\alpha_{0}, \alpha_{1},\|g\|_{L_{\infty}},\left\|g^{-1}\right\|_{L_{\infty}}$, and the parameters of the lattice $\Gamma$.

Now we estimate the $\left(L_{2} \rightarrow L_{2}\right)$-norm of the corrector. By the scaling transformation,

$$
\begin{aligned}
& \varepsilon\left\|\Lambda^{\varepsilon} b(\mathbf{D}) \Pi_{\varepsilon} e^{-\widehat{\mathcal{A}}^{0} \tau}\right\|_{L_{2}\left(\mathbb{R}^{d}\right) \rightarrow L_{2}\left(\mathbb{R}^{d}\right)}=\left\|\Lambda b(\mathbf{D}) \Pi e^{-\widehat{\mathcal{A}}^{0} \tau \varepsilon^{-2}}\right\|_{L_{2}\left(\mathbb{R}^{d}\right) \rightarrow L_{2}\left(\mathbb{R}^{d}\right)} \\
& \leq\left\|\Lambda \Pi_{m}\right\|_{L_{2}\left(\mathbb{R}^{d}\right) \rightarrow L_{2}\left(\mathbb{R}^{d}\right)}\left\|b(\mathbf{D}) e^{-\widehat{\mathcal{A}}^{0} \tau \varepsilon^{-2}}\right\|_{L_{2}\left(\mathbb{R}^{d}\right) \rightarrow L_{2}\left(\mathbb{R}^{d}\right)} .
\end{aligned}
$$

Here $\Pi_{m}$ is a pseudodifferential operator with the symbol $\chi_{\widetilde{\Omega} / \varepsilon}(\boldsymbol{\xi})$ acting in $L_{2}\left(\mathbb{R}^{d} ; \mathbb{C}^{m}\right)$. By (5.2) and (6.16), we have

$$
\begin{aligned}
& \left\|b(\mathbf{D}) e^{-\widehat{\mathcal{A}}^{0} \tau \varepsilon^{-2}}\right\|_{L_{2}\left(\mathbb{R}^{d}\right) \rightarrow L_{2}\left(\mathbb{R}^{d}\right)} \leq \sup _{\boldsymbol{\xi} \in \mathbb{R}^{d}}\left|b(\boldsymbol{\xi}) \exp \left(-b(\boldsymbol{\xi})^{*} g^{0} b(\boldsymbol{\xi}) \tau \varepsilon^{-2}\right)\right| \\
& \quad \leq \alpha_{1}^{1 / 2} \sup _{\boldsymbol{\xi} \in \mathbb{R}^{d}}|\boldsymbol{\xi}| \exp \left(-\widehat{c}_{*}|\boldsymbol{\xi}|^{2} \tau \varepsilon^{-2}\right) \leq \varepsilon \tau^{-1 / 2} \alpha_{1}^{1 / 2}\left(\widehat{c}_{*}\right)^{-1 / 2}, \quad \tau>0, \quad \varepsilon>0 .
\end{aligned}
$$

Using the Gelfand transformation and (7.37), we estimate the norm of the operator $\Lambda \Pi_{m}$ :

$$
\left\|\Lambda \Pi_{m}\right\|_{L_{2}\left(\mathbb{R}^{d}\right) \rightarrow L_{2}\left(\mathbb{R}^{d}\right)}=\left\|\Lambda \widehat{P}_{m}\right\|_{L_{2}(\Omega) \rightarrow L_{2}(\Omega)} \leq m^{1 / 2}\left(2 r_{0}\right)^{-1} \alpha_{0}^{-1 / 2}\|g\|_{L_{\infty}}^{1 / 2}\left\|g^{-1}\right\|_{L_{\infty}}^{1 / 2} .
$$

Relations (11.6)-(11.9) imply that

$$
\left\|e^{-\widehat{\mathcal{A}}_{\varepsilon} \tau}-\left(I+\varepsilon \Lambda^{\varepsilon} b(\mathbf{D}) \Pi_{\varepsilon}\right) e^{-\widehat{\mathcal{A}}^{0} \tau}\right\|_{L_{2}\left(\mathbb{R}^{d}\right) \rightarrow L_{2}\left(\mathbb{R}^{d}\right)} \leq \mathcal{C}_{9} \tau^{-1 / 2} \varepsilon, \quad \tau>0, \quad \varepsilon>0,
$$


where

$$
\mathcal{C}_{9}=\widehat{\mathcal{C}}_{0}+m^{1 / 2}\left(2 r_{0}\right)^{-1} \alpha_{0}^{-1 / 2} \alpha_{1}^{1 / 2}\left(\widehat{c}_{*}\right)^{-1 / 2}\|g\|_{L_{\infty}}^{1 / 2}\left\|g^{-1}\right\|_{L_{\infty}}^{1 / 2}
$$

Inequalities (11.5) and (11.10) lead to the following result.

Theorem 11.1. Let $\widehat{\mathcal{A}}_{\varepsilon}=b(\mathbf{D})^{*} g^{\varepsilon}(\mathbf{x}) b(\mathbf{D})$, and let $\widehat{\mathcal{A}}^{0}=b(\mathbf{D})^{*} g^{0} b(\mathbf{D})$ be the effective operator. Let $\Lambda(\mathbf{x})$ be the $\Gamma$-periodic solution of problem $(6.11)$, and let $\Lambda^{\varepsilon}(\mathbf{x})=\Lambda\left(\varepsilon^{-1} \mathbf{x}\right)$. Let $\Pi_{\varepsilon}$ be the operator (11.1). Then for $\tau>0$ and $\varepsilon>0$ we have

$$
\begin{gathered}
\left\|e^{-\widehat{\mathcal{A}}_{\varepsilon} \tau}-\left(I+\varepsilon \Lambda^{\varepsilon} b(\mathbf{D}) \Pi_{\varepsilon}\right) e^{-\widehat{\mathcal{A}}^{0} \tau}\right\|_{L_{2}\left(\mathbb{R}^{d}\right) \rightarrow H^{1}\left(\mathbb{R}^{d}\right)} \leq \Psi_{1}(\tau, \varepsilon), \\
\Psi_{1}(\tau, \varepsilon)=\left\{\begin{aligned}
\varepsilon\left(\check{\mathcal{C}}_{1} \tau^{-1}+\mathcal{C}_{9} \tau^{-1 / 2}\right), & \tau>0, \quad 0<\varepsilon \leq \tau^{1 / 2}, \\
\tau^{-1 / 2}\left(\check{\mathcal{C}}_{2}+\mathcal{C}_{9} \varepsilon\right), & \tau>0, \quad \varepsilon>\tau^{1 / 2} .
\end{aligned}\right.
\end{gathered}
$$

The constants $\check{\mathcal{C}}_{1}=\left(\widehat{\mathcal{C}}_{*}\right)^{-1 / 2} \mathcal{C}_{1}, \check{\mathcal{C}}_{2}=\left(\widehat{\mathcal{C}}_{*}\right)^{-1 / 2} \mathcal{C}_{2}$ and $\mathcal{C}_{9}$ depend only on $m, \alpha_{0}, \alpha_{1},\|g\|_{L_{\infty}}$, $\left\|g^{-1}\right\|_{L_{\infty}}$, and the parameters of the lattice $\Gamma$.

\subsection{Approximation of the operator $\exp \left(-\widehat{\mathcal{A}}_{\varepsilon} \tau\right)$ for $\varepsilon \leq \tau^{1 / 2}$}

Now we apply Theorem 9.4. Similarly to the proof of (11.3), by the scaling transformation, (9.17) implies that

$$
\left\|\widehat{\mathcal{A}}_{\varepsilon}^{1 / 2}\left(e^{-\widehat{\mathcal{A}}_{\varepsilon} \tau}-\left(I+\varepsilon \Lambda^{\varepsilon} b(\mathbf{D})\right) e^{-\widehat{\mathcal{A}}^{0} \tau}\right)\right\|_{L_{2}\left(\mathbb{R}^{d}\right) \rightarrow L_{2}\left(\mathbb{R}^{d}\right)} \leq \mathcal{C}_{1}^{\prime} \tau^{-1} \varepsilon, \quad \tau>0, \quad 0<\varepsilon \leq \tau^{1 / 2} .
$$

Combining this with (11.4), we obtain

$$
\left\|\mathbf{D}\left(e^{-\widehat{\mathcal{A}}_{\varepsilon} \tau}-\left(I+\varepsilon \Lambda^{\varepsilon} b(\mathbf{D})\right) e^{-\widehat{\mathcal{A}}^{0} \tau}\right)\right\|_{L_{2}\left(\mathbb{R}^{d}\right) \rightarrow L_{2}\left(\mathbb{R}^{d}\right)} \leq\left(\widehat{c}_{*}\right)^{-1 / 2} \mathcal{C}_{1}^{\prime} \tau^{-1} \varepsilon, \quad \tau>0, \quad 0<\varepsilon \leq \tau^{1 / 2} .
$$

Let us estimate the $\left(L_{2} \rightarrow L_{2}\right)$-norm of the corrector. By the scaling transformation,

$$
\varepsilon\left\|\Lambda^{\varepsilon} b(\mathbf{D}) e^{-\widehat{\mathcal{A}}^{0} \tau}\right\|_{L_{2}\left(\mathbb{R}^{d}\right) \rightarrow L_{2}\left(\mathbb{R}^{d}\right)}=\left\|\Lambda b(\mathbf{D}) e^{-\widehat{\mathcal{A}}^{0} \tau \varepsilon^{-2}}\right\|_{L_{2}\left(\mathbb{R}^{d}\right) \rightarrow L_{2}\left(\mathbb{R}^{d}\right)} .
$$

We need to estimate the operator $b(\mathbf{D}) e^{-\widehat{\mathcal{A}}^{0} \tau}$ in the $\left(L_{2} \rightarrow H^{s}\right)$-norm and the operator $[\Lambda]$ in the $\left(H^{s} \rightarrow L_{2}\right)$-norm (with appropriate $s$ ).

Proposition 11.2. For $\tau \geq 1$ the operator $b(\mathbf{D}) e^{-\widehat{\mathcal{A}}^{0} \tau}$ is a continuous mapping of $L_{2}\left(\mathbb{R}^{d} ; \mathbb{C}^{n}\right)$ to $H^{s}\left(\mathbb{R}^{d} ; \mathbb{C}^{m}\right)$ with any $s \geq 0$, and

$$
\left\|b(\mathbf{D}) e^{-\widehat{\mathcal{A}}^{0} \tau}\right\|_{L_{2}\left(\mathbb{R}^{d}\right) \rightarrow H^{s}\left(\mathbb{R}^{d}\right)} \leq C_{s}^{\circ} \tau^{-1 / 2}, \quad \tau \geq 1 .
$$

The constant $C_{s}^{\circ}$ is defined below in (11.17) and depends only on $s, \alpha_{0}, \alpha_{1},\left\|g^{-1}\right\|_{L_{\infty}}$.

Proof. By (5.2) and (6.16), we have

$$
\begin{aligned}
\left\|b(\mathbf{D}) e^{-\widehat{\mathcal{A}}^{0} \tau}\right\|_{L_{2}\left(\mathbb{R}^{d}\right) \rightarrow H^{s}\left(\mathbb{R}^{d}\right)} & \leq \sup _{\boldsymbol{\xi} \in \mathbb{R}^{d}}\left(1+|\boldsymbol{\xi}|^{2}\right)^{s / 2}\left|b(\boldsymbol{\xi}) \exp \left(-b(\boldsymbol{\xi})^{*} g^{0} b(\boldsymbol{\xi}) \tau\right)\right| \\
& \leq \alpha_{1}^{1 / 2} \sup _{\boldsymbol{\xi} \in \mathbb{R}^{d}}\left(1+|\boldsymbol{\xi}|^{2}\right)^{s / 2}|\boldsymbol{\xi}| \exp \left(-\widehat{c}_{*}|\boldsymbol{\xi}|^{2} \tau\right) .
\end{aligned}
$$


We denote $\widetilde{c}_{s}=\sup _{\alpha>0}(1+\alpha)^{s / 2} \alpha^{1 / 2} e^{-\alpha}$. Then for $\tau \geq 1$ we obtain

$$
\begin{aligned}
\left\|b(\mathbf{D}) e^{-\widehat{\mathcal{A}}^{0} \tau}\right\|_{L_{2}\left(\mathbb{R}^{d}\right) \rightarrow H^{s}\left(\mathbb{R}^{d}\right)} & \leq \widetilde{c}_{s} \alpha_{1}^{1 / 2} \sup _{\boldsymbol{\xi} \in \mathbb{R}^{d}}\left(\frac{1+|\boldsymbol{\xi}|^{2}}{1+\widehat{c}_{*}|\boldsymbol{\xi}|^{2}}\right)^{s / 2} \frac{|\boldsymbol{\xi}|}{\left(\widehat{c}_{*}\right)^{1 / 2}|\boldsymbol{\xi}| \tau^{1 / 2}} \\
& \leq \tau^{-1 / 2} \widetilde{c}_{s} \alpha_{1}^{1 / 2}\left(\widehat{c}_{*}\right)^{-1 / 2}\left(1+\left(\widehat{c}_{*}\right)^{-1}\right)^{s / 2}, \quad \tau \geq 1 .
\end{aligned}
$$

Thus, inequality (11.16) is valid with the constant

$$
C_{s}^{\circ}=\widetilde{c}_{s} \alpha_{1}^{1 / 2}\left(\widehat{c}_{*}\right)^{-1 / 2}\left(1+\left(\widehat{c}_{*}\right)^{-1}\right)^{s / 2} .
$$

Proposition 11.3. Let $s=0$ for $d=1, s>0$ for $d=2, s=d / 2-1$ for $d \geq 3$. Then the operator $[\Lambda]$ of multiplication by the matrix-valued function $\Lambda$ is a continuous mapping of $H^{s}\left(\mathbb{R}^{d} ; \mathbb{C}^{m}\right)$ to $L_{2}\left(\mathbb{R}^{d} ; \mathbb{C}^{n}\right)$, and

$$
\|[\Lambda]\|_{H^{s}\left(\mathbb{R}^{d}\right) \rightarrow L_{2}\left(\mathbb{R}^{d}\right)} \leq \widetilde{\mathfrak{C}}_{d}
$$

where the constant $\widetilde{\mathfrak{C}}_{d}$ depends only on $d, m, n, \alpha_{0},\|g\|_{L_{\infty}},\left\|g^{-1}\right\|_{L_{\infty}}$, and the parameters of the lattice $\Gamma$; in the case $d=2$ it depends also on $s$.

Proof. Let us assume that $0<s<1$ in the case $d=2$. Consider the operators $\left[\mathbf{v}_{j}\right]$ of multiplication by the vector-valued functions $\mathbf{v}_{j}(\mathbf{x})$ (the columns of $\Lambda(\mathbf{x})$ ). It suffices to estimate the norm of $\left[\mathbf{v}_{j}\right]$ as an operator from $H^{s}\left(\mathbb{R}^{d}\right)$ to $L_{2}\left(\mathbb{R}^{d} ; \mathbb{C}^{n}\right)$, for all $j=1, \ldots, m$. Let $c(d, n)$ be the norm of the embedding $H^{1}\left(\Omega ; \mathbb{C}^{n}\right) \subset L_{q}\left(\Omega ; \mathbb{C}^{n}\right)$, where $q=\infty$ for $d=1$, $q=2 / s$ for $d=2, q=2 d /(d-2)$ for $d \geq 3$. By $\check{c}_{d}$ we denote the norm of the embedding $H^{s}(\Omega) \subset L_{p}(\Omega)$, where $s=0$ and $p=2$ for $d=1 ; 0<s<1$ and $p=2 /(1-s)$ for $d=2$; $s=d / 2-1$ and $p=d$ for $d \geq 3$. Similarly to (9.12)-(9.16), we obtain

$$
\int_{\mathbb{R}^{d}}\left|\mathbf{v}_{j}(\mathbf{x})\right|^{2}|u|^{2} d \mathbf{x} \leq c(d, n)^{2} \check{c}_{d}^{2}\left\|\mathbf{v}_{j}\right\|_{H^{1}(\Omega)}^{2}\|u\|_{H^{s}\left(\mathbb{R}^{d}\right)}^{2} .
$$

Combining this with estimate (9.6), we obtain

$$
\left\|\left[v_{j}\right]\right\|_{H^{s}\left(\mathbb{R}^{d}\right) \rightarrow L_{2}\left(\mathbb{R}^{d}\right)} \leq c(d, n) \check{c}_{d}\left(1+1 / 4 r_{0}^{2}\right)^{1 / 2} \alpha_{0}^{-1 / 2}|\Omega|^{1 / 2}\|g\|_{L_{\infty}}^{1 / 2}\left\|g^{-1}\right\|_{L_{\infty}}^{1 / 2}=: \widetilde{\mathfrak{C}}_{d}^{\prime}, \quad j=1, \ldots, m .
$$

Estimate (11.19) implies (11.18) with $\widetilde{\mathfrak{C}}_{d}=m^{1 / 2} \widetilde{\mathfrak{C}}_{d}^{\prime}$.

Let $s$ be as in Proposition 11.3. In the case $d=2$, we fix $s$ (for instance, $s=1 / 2$ ). From (11.16) and (11.18) it follows that

$$
\left\|\Lambda b(\mathbf{D}) e^{-\widehat{\mathcal{A}}^{0} \tau}\right\|_{L_{2}\left(\mathbb{R}^{d}\right) \rightarrow L_{2}\left(\mathbb{R}^{d}\right)} \leq C_{s}^{\circ} \widetilde{\mathfrak{C}}_{d}^{\tau^{-1 / 2}}, \quad \tau \geq 1
$$

Relations (11.15) and (11.20) imply that

$$
\varepsilon\left\|\Lambda^{\varepsilon} b(\mathbf{D}) e^{-\widehat{\mathcal{A}}^{0} \tau}\right\|_{L_{2}\left(\mathbb{R}^{d}\right) \rightarrow L_{2}\left(\mathbb{R}^{d}\right)} \leq C_{s}^{\circ} \widetilde{\mathfrak{C}}_{d} \tau^{-1 / 2} \varepsilon, \quad \tau>0, \quad 0<\varepsilon \leq \tau^{1 / 2}
$$


The following result is a direct consequence of (11.14), (11.6), and (11.21).

Theorem 11.4. Suppose that the assumptions of Theorem 11.1 are satisfied. Then we have $\left\|e^{-\widehat{\mathcal{A}}_{\varepsilon} \tau}-\left(I+\varepsilon \Lambda^{\varepsilon} b(\mathbf{D})\right) e^{-\widehat{\mathcal{A}}^{0} \tau}\right\|_{L_{2}\left(\mathbb{R}^{d}\right) \rightarrow H^{1}\left(\mathbb{R}^{d}\right)} \leq \varepsilon\left(\mathcal{C}_{10}^{(1)} \tau^{-1}+\mathcal{C}_{10}^{(2)} \tau^{-1 / 2}\right), \quad \tau>0, \quad 0<\varepsilon \leq \tau^{1 / 2}$.

The constants $\mathcal{C}_{10}^{(1)}=\left(\widehat{c}_{*}\right)^{-1 / 2} \mathcal{C}_{1}^{\prime}, \mathcal{C}_{10}^{(2)}=\widehat{\mathcal{C}}_{0}+C_{s}^{\circ} \widetilde{\mathfrak{C}}_{d}$ depend only on $d, m, n, \alpha_{0}, \alpha_{1},\|g\|_{L_{\infty}}$, $\left\|g^{-1}\right\|_{L_{\infty}}$, and the parameters of the lattice $\Gamma$.

\subsection{Approximation of the operator $g^{\varepsilon} b(\mathbf{D}) \exp \left(-\widehat{\mathcal{A}}_{\varepsilon} \tau\right)$}

By the scaling transformation, Theorem 9.5 implies the following result.

Theorem 11.5. Suppose that the assumptions of Theorem 11.1 are satisfied. Let $\widetilde{g}(\mathbf{x})$ be the matrix (6.12), and let $\widetilde{g}^{\varepsilon}(\mathbf{x})=\widetilde{g}\left(\varepsilon^{-1} \mathbf{x}\right)$. Then for $\tau>0$ and $\varepsilon>0$ we have

$$
\begin{gathered}
\left\|g^{\varepsilon} b(\mathbf{D}) e^{-\widehat{\mathcal{A}}_{\varepsilon} \tau}-\widetilde{g}^{\varepsilon} b(\mathbf{D}) e^{-\widehat{\mathcal{A}}^{0} \tau} \Pi_{\varepsilon}\right\|_{L_{2}\left(\mathbb{R}^{d}\right) \rightarrow L_{2}\left(\mathbb{R}^{d}\right)} \leq \Sigma_{1}(\tau, \varepsilon), \\
\Sigma_{1}(\tau, \varepsilon)=\left\{\begin{array}{lc}
\mathcal{C}_{3} \tau^{-1} \varepsilon, & \tau>0, \quad 0<\varepsilon \leq \tau^{1 / 2} \\
\mathcal{C}_{4} \tau^{-1 / 2}, & \tau>0, \quad \varepsilon>\tau^{1 / 2}
\end{array}\right.
\end{gathered}
$$

The constants $\mathcal{C}_{3}, \mathcal{C}_{4}$ depend only on $d, m, \alpha_{0}, \alpha_{1},\|g\|_{L_{\infty}},\left\|g^{-1}\right\|_{L_{\infty}}$, and the parameters of the lattice $\Gamma$.

Similarly, Theorem 9.7 implies the following statement.

Theorem 11.6. Under the assumptions of Theorem 11.5, we have

$$
\left\|g^{\varepsilon} b(\mathbf{D}) e^{-\widehat{\mathcal{A}}_{\varepsilon} \tau}-\widetilde{g}^{\varepsilon} b(\mathbf{D}) e^{-\widehat{\mathcal{A}}^{0} \tau}\right\|_{L_{2}\left(\mathbb{R}^{d}\right) \rightarrow L_{2}\left(\mathbb{R}^{d}\right)} \leq \mathcal{C}_{3}^{\prime} \tau^{-1} \varepsilon, \quad \tau>0, \quad 0<\varepsilon \leq \tau^{1 / 2} .
$$

The constant $\mathcal{C}_{3}^{\prime}$ depends only on $d, m, n, \alpha_{0}, \alpha_{1},\|g\|_{L_{\infty}},\left\|g^{-1}\right\|_{L_{\infty}}$, and the parameters of the lattice $\Gamma$.

\subsection{Special cases}

Suppose that condition (7.41) is satisfied. Then, by the scaling transformation, from (9.22) it follows that

$$
\begin{gathered}
\left\|\widehat{\mathcal{A}}_{\varepsilon}^{1 / 2}\left(e^{-\widehat{\mathcal{A}}_{\varepsilon} \tau}-e^{-\widehat{\mathcal{A}}^{0} \tau}\right)\right\|_{L_{2}\left(\mathbb{R}^{d}\right) \rightarrow L_{2}\left(\mathbb{R}^{d}\right)} \leq \widetilde{\Psi}_{1}^{0}(\tau, \varepsilon), \quad \tau>0, \quad \varepsilon>0, \\
\widetilde{\Psi}_{1}^{0}(\tau, \varepsilon)=\left\{\begin{array}{c}
\mathcal{C}_{1}^{0} \tau^{-1} \varepsilon, \quad \tau>0, \quad 0<\varepsilon \leq \tau^{1 / 2} \\
\mathcal{C}_{2}^{0} \tau^{-1 / 2}, \quad \tau>0, \quad \varepsilon>\tau^{1 / 2}
\end{array}\right.
\end{gathered}
$$

Combining this with (11.4), we obtain

$$
\left\|\mathbf{D}\left(e^{-\widehat{\mathcal{A}}_{\varepsilon} \tau}-e^{-\widehat{\mathcal{A}}^{0} \tau}\right)\right\|_{L_{2}\left(\mathbb{R}^{d}\right) \rightarrow L_{2}\left(\mathbb{R}^{d}\right)} \leq\left(\widehat{c}_{*}\right)^{-1 / 2} \widetilde{\Psi}_{1}^{0}(\tau, \varepsilon), \quad \tau>0, \quad \varepsilon>0 .
$$


Together with (11.6) this leads to the following result.

Proposition 11.7. Suppose that the assumptions of Theorem 11.1 and also condition (7.41) are satisfied. Then for $\tau>0$ and $\varepsilon>0$ we have

$$
\begin{gathered}
\left\|e^{-\widehat{\mathcal{A}}_{\varepsilon} \tau}-e^{-\widehat{\mathcal{A}}^{0} \tau}\right\|_{L_{2}\left(\mathbb{R}^{d}\right) \rightarrow H^{1}\left(\mathbb{R}^{d}\right)} \leq \Psi_{1}^{0}(\tau, \varepsilon), \\
\Psi_{1}^{0}(\tau, \varepsilon)=\left\{\begin{aligned}
\varepsilon\left(\check{\mathcal{C}}_{1}^{0} \tau^{-1}+\widehat{\mathcal{C}}_{0} \tau^{-1 / 2}\right), & \tau>0, \quad 0<\varepsilon \leq \tau^{1 / 2}, \\
\tau^{-1 / 2}\left(\check{\mathcal{C}}_{2}^{0}+\widehat{\mathcal{C}}_{0} \varepsilon\right), & \tau>0, \quad \varepsilon>\tau^{1 / 2} .
\end{aligned}\right.
\end{gathered}
$$

The constants $\breve{\mathcal{C}}_{1}^{0}=\left(\widehat{\mathcal{C}}_{*}\right)^{-1 / 2} \mathcal{C}_{1}^{0}, \check{\mathcal{C}}_{2}^{0}=\left(\widehat{\mathcal{C}}_{*}\right)^{-1 / 2} \mathcal{C}_{2}^{0}, \widehat{\mathcal{C}}_{0}$ depend only on $\alpha_{0}, \alpha_{1},\|g\|_{L_{\infty}},\left\|g^{-1}\right\|_{L_{\infty}}$, and the parameters of the lattice $\Gamma$.

Finally, by the scaling transformation, Proposition 9.9 implies the following result.

Proposition 11.8. Suppose that the assumptions of Theorem 11.1 and also condition (6.20) are satisfied. Then for $\tau>0$ and $\varepsilon>0$ we have

$$
\begin{gathered}
\left\|g^{\varepsilon} b(\mathbf{D}) e^{-\widehat{\mathcal{A}}_{\varepsilon} \tau}-g^{0} b(\mathbf{D}) e^{-\widehat{\mathcal{A}}^{0} \tau}\right\|_{L_{2}\left(\mathbb{R}^{d}\right) \rightarrow L_{2}\left(\mathbb{R}^{d}\right)} \leq \Sigma_{1}^{0}(\tau, \varepsilon), \\
\Sigma_{1}^{0}(\tau, \varepsilon)=\left\{\begin{array}{c}
\mathcal{C}_{3}^{0} \tau^{-1} \varepsilon, \quad \tau>0, \quad 0<\varepsilon \leq \tau^{1 / 2}, \\
\mathcal{C}_{4}^{0} \tau^{-1 / 2}, \quad \tau>0, \quad \varepsilon>\tau^{1 / 2}
\end{array}\right.
\end{gathered}
$$

The constant $\mathcal{C}_{4}^{0}$ depends only on $\alpha_{0}, \alpha_{1},\|g\|_{L_{\infty}},\left\|g^{-1}\right\|_{L_{\infty}} ;$ the constant $\mathcal{C}_{3}^{0}$ depends on the same parameters and on $d, m$, and the parameters of the lattice $\Gamma$.

\section{Approximation of the bordered operator $e^{-\mathcal{A}_{\varepsilon} \tau}$}

\subsection{Main theorem}

By the scaling transformation, Theorem 10.1 implies the estimate

$$
\begin{gathered}
\left\|\widehat{\mathcal{A}}_{\varepsilon}^{1 / 2}\left(f^{\varepsilon} e^{-\mathcal{A}_{\varepsilon} \tau}\left(f^{\varepsilon}\right)^{*}-\left(I+\varepsilon \Lambda^{\varepsilon} b(\mathbf{D}) \Pi_{\varepsilon}\right) f_{0} e^{-\mathcal{A}^{0} \tau} f_{0}\right)\right\|_{L_{2}\left(\mathbb{R}^{d}\right) \rightarrow L_{2}\left(\mathbb{R}^{d}\right)} \leq \widetilde{\Psi}_{2}(\tau, \varepsilon), \\
\widetilde{\Psi}_{2}(\tau, \varepsilon)=\left\{\begin{array}{cc}
\mathcal{C}_{5} \tau^{-1} \varepsilon, & \tau>0, \quad 0<\varepsilon \leq \tau^{1 / 2}, \\
\mathcal{C}_{6} \tau^{-1 / 2}, & \tau>0, \quad \varepsilon>\tau^{1 / 2}
\end{array}\right.
\end{gathered}
$$

Combining this with (11.4), for $\tau>0$ and $\varepsilon>0$ we obtain

$$
\left\|\mathbf{D}\left(f^{\varepsilon} e^{-\mathcal{A}_{\varepsilon} \tau}\left(f^{\varepsilon}\right)^{*}-\left(I+\varepsilon \Lambda^{\varepsilon} b(\mathbf{D}) \Pi_{\varepsilon}\right) f_{0} e^{-\mathcal{A}^{0} \tau} f_{0}\right)\right\|_{L_{2}\left(\mathbb{R}^{d}\right) \rightarrow L_{2}\left(\mathbb{R}^{d}\right)} \leq\left(\widehat{c}_{*}\right)^{-1 / 2} \widetilde{\Psi}_{2}(\tau, \varepsilon) .
$$

According to [Su2, Theorem 7.3], we have

$$
\left\|f^{\varepsilon} e^{-\mathcal{A}_{\varepsilon} \tau}\left(f^{\varepsilon}\right)^{*}-f_{0} e^{-\mathcal{A}^{0} \tau} f_{0}\right\|_{L_{2}\left(\mathbb{R}^{d}\right) \rightarrow L_{2}\left(\mathbb{R}^{d}\right)} \leq \mathcal{C}_{0} \tau^{-1 / 2} \varepsilon, \quad \tau>0, \quad \varepsilon>0,
$$


where the constant $\mathcal{C}_{0}$ depends only on $\alpha_{0}, \alpha_{1},\|g\|_{L_{\infty}},\left\|g^{-1}\right\|_{L_{\infty}},\|f\|_{L_{\infty}},\left\|f^{-1}\right\|_{L_{\infty}}$, and the parameters of the lattice $\Gamma$.

Let us estimate the $\left(L_{2} \rightarrow L_{2}\right)$-norm of the corrector. By the scaling transformation,

$$
\begin{aligned}
& \varepsilon\left\|\Lambda^{\varepsilon} b(\mathbf{D}) \Pi_{\varepsilon} f_{0} e^{-\mathcal{A}^{0} \tau} f_{0}\right\|_{L_{2}\left(\mathbb{R}^{d}\right) \rightarrow L_{2}\left(\mathbb{R}^{d}\right)}=\left\|\Lambda b(\mathbf{D}) \Pi f_{0} e^{-\mathcal{A}^{0} \tau \varepsilon^{-2}} f_{0}\right\|_{L_{2}\left(\mathbb{R}^{d}\right) \rightarrow L_{2}\left(\mathbb{R}^{d}\right)} \\
& \leq\left\|\Lambda \Pi_{m}\right\|_{L_{2}\left(\mathbb{R}^{d}\right) \rightarrow L_{2}\left(\mathbb{R}^{d}\right)}\left\|b(\mathbf{D}) f_{0} e^{-\mathcal{A}^{0} \tau \varepsilon^{-2}} f_{0}\right\|_{L_{2}\left(\mathbb{R}^{d}\right) \rightarrow L_{2}\left(\mathbb{R}^{d}\right)} .
\end{aligned}
$$

Similarly to (11.8), taking (5.2), (8.3), and (8.6) into account, we obtain

$$
\begin{aligned}
& \left\|b(\mathbf{D}) f_{0} e^{-\mathcal{A}^{0} \tau \varepsilon^{-2}} f_{0}\right\|_{L_{2}\left(\mathbb{R}^{d}\right) \rightarrow L_{2}\left(\mathbb{R}^{d}\right)} \leq \alpha_{1}^{1 / 2}\|f\|_{L_{\infty}}^{2} \sup _{\boldsymbol{\xi} \in \mathbb{R}^{d}}|\boldsymbol{\xi}| \exp \left(-c_{*}|\boldsymbol{\xi}|^{2} \tau \varepsilon^{-2}\right) \\
& \quad \leq \varepsilon \tau^{-1 / 2} \alpha_{1}^{1 / 2}\|f\|_{L_{\infty}}^{2} c_{*}^{-1 / 2}, \quad \tau>0, \quad \varepsilon>0 .
\end{aligned}
$$

Relations (12.2)-(12.4) and (11.9) imply the estimate

$$
\left\|f^{\varepsilon} e^{-\mathcal{A}_{\varepsilon} \tau}\left(f^{\varepsilon}\right)^{*}-\left(I+\varepsilon \Lambda^{\varepsilon} b(\mathbf{D}) \Pi_{\varepsilon}\right) f_{0} e^{-\mathcal{A}^{0} \tau} f_{0}\right\|_{L_{2}\left(\mathbb{R}^{d}\right) \rightarrow L_{2}\left(\mathbb{R}^{d}\right)} \leq \mathcal{C}_{11} \tau^{-1 / 2} \varepsilon, \quad \tau>0, \quad \varepsilon>0,
$$

where

$$
\mathcal{C}_{11}=\mathcal{C}_{0}+m^{1 / 2}\left(2 r_{0}\right)^{-1} \alpha_{0}^{-1 / 2} \alpha_{1}^{1 / 2}\|f\|_{L_{\infty}}^{2}\|g\|_{L_{\infty}}^{1 / 2}\left\|g^{-1}\right\|_{L_{\infty}}^{1 / 2} c_{*}^{-1 / 2}
$$

The next theorem follows from (12.1) and (12.5).

Theorem 12.1. Let $\mathcal{A}_{\varepsilon}=\left(f^{\varepsilon}\right)^{*} b(\mathbf{D})^{*} g^{\varepsilon}(\mathbf{x}) b(\mathbf{D}) f^{\varepsilon}$, and let $\mathcal{A}^{0}=f_{0} b(\mathbf{D})^{*} g^{0} b(\mathbf{D}) f_{0}$, where $g^{0}$ is defined by (6.13), and $f_{0}$ is defined by (8.2). Let $\Lambda(\mathbf{x})$ be the $\Gamma$-periodic solution of equation (6.11), and let $\Lambda^{\varepsilon}(\mathbf{x})=\Lambda\left(\varepsilon^{-1} \mathbf{x}\right)$. Let $\Pi_{\varepsilon}$ be the operator (11.1). Then for $\tau>0$ and $\varepsilon>0$ we have

$$
\begin{gathered}
\left\|f^{\varepsilon} e^{-\mathcal{A}_{\varepsilon} \tau}\left(f^{\varepsilon}\right)^{*}-\left(I+\varepsilon \Lambda^{\varepsilon} b(\mathbf{D}) \Pi_{\varepsilon}\right) f_{0} e^{-\mathcal{A}^{0} \tau} f_{0}\right\|_{L_{2}\left(\mathbb{R}^{d}\right) \rightarrow H^{1}\left(\mathbb{R}^{d}\right)} \leq \Psi_{2}(\tau, \varepsilon), \\
\Psi_{2}(\tau, \varepsilon)=\left\{\begin{array}{cc}
\varepsilon\left(\check{\mathcal{C}}_{5} \tau^{-1}+\mathcal{C}_{11} \tau^{-1 / 2}\right), & \tau>0, \quad 0<\varepsilon \leq \tau^{1 / 2}, \\
\tau^{-1 / 2}\left(\check{\mathcal{C}}_{6}+\mathcal{C}_{11} \varepsilon\right), & \tau>0, \quad \varepsilon>\tau^{1 / 2}
\end{array}\right.
\end{gathered}
$$

The constants $\check{\mathcal{C}}_{5}=\left(\widehat{c}_{*}\right)^{-1 / 2} \mathcal{C}_{5}, \check{\mathcal{C}}_{6}=\left(\widehat{c}_{*}\right)^{-1 / 2} \mathcal{C}_{6}$, and $\mathcal{C}_{11}$ depend only on $m, \alpha_{0}, \alpha_{1},\|g\|_{L_{\infty}}$, $\left\|g^{-1}\right\|_{L_{\infty}},\|f\|_{L_{\infty}},\left\|f^{-1}\right\|_{L_{\infty}}$, and the parameters of the lattice $\Gamma$.

\subsection{Approximation of the operator $f^{\varepsilon} e^{-\mathcal{A}_{\varepsilon} \tau}\left(f^{\varepsilon}\right)^{*}$ for $\varepsilon \leq \tau^{1 / 2}$}

Now we apply Theorem 10.3. By the scaling transformation, (10.1) implies that

$$
\begin{array}{r}
\left\|\widehat{\mathcal{A}}_{\varepsilon}^{1 / 2}\left(f^{\varepsilon} e^{-\mathcal{A}_{\varepsilon} \tau}\left(f^{\varepsilon}\right)^{*}-\left(I+\varepsilon \Lambda^{\varepsilon} b(\mathbf{D})\right) f_{0} e^{-\mathcal{A}^{0} \tau} f_{0}\right)\right\|_{L_{2}\left(\mathbb{R}^{d}\right) \rightarrow L_{2}\left(\mathbb{R}^{d}\right)} \leq \mathcal{C}_{5}^{\prime} \tau^{-1} \varepsilon \\
\tau>0, \quad 0<\varepsilon \leq \tau^{1 / 2}
\end{array}
$$

Combining this with (11.4), we see that

$$
\begin{array}{r}
\left\|\mathbf{D}\left(f^{\varepsilon} e^{-\mathcal{A}_{\varepsilon} \tau}\left(f^{\varepsilon}\right)^{*}-\left(I+\varepsilon \Lambda^{\varepsilon} b(\mathbf{D})\right) f_{0} e^{-\mathcal{A}^{0} \tau} f_{0}\right)\right\|_{L_{2}\left(\mathbb{R}^{d}\right) \rightarrow L_{2}\left(\mathbb{R}^{d}\right)} \leq\left(\widehat{c}_{*}\right)^{-1 / 2} \mathcal{C}_{5}^{\prime} \tau^{-1} \varepsilon \\
\tau>0, \quad 0<\varepsilon \leq \tau^{1 / 2}
\end{array}
$$


Let us estimate the $\left(L_{2} \rightarrow L_{2}\right)$-norm of the corrector. By the scaling transformation,

$$
\varepsilon\left\|\Lambda^{\varepsilon} b(\mathbf{D}) f_{0} e^{-\mathcal{A}^{0} \tau} f_{0}\right\|_{L_{2}\left(\mathbb{R}^{d}\right) \rightarrow L_{2}\left(\mathbb{R}^{d}\right)}=\left\|\Lambda b(\mathbf{D}) f_{0} e^{-\mathcal{A}^{0} \tau \varepsilon^{-2}} f_{0}\right\|_{L_{2}\left(\mathbb{R}^{d}\right) \rightarrow L_{2}\left(\mathbb{R}^{d}\right)} .
$$

It is easy to check the following statement by analogy with Proposition 11.2.

Proposition 12.2. For $\tau \geq 1$ the operator $b(\mathbf{D}) f_{0} e^{-\mathcal{A}^{0} \tau} f_{0}$ is a continuous mapping of $L_{2}\left(\mathbb{R}^{d} ; \mathbb{C}^{n}\right)$ to $H^{s}\left(\mathbb{R}^{d} ; \mathbb{C}^{m}\right)$ for any $s \geq 0$, and

$$
\left\|b(\mathbf{D}) f_{0} e^{-\mathcal{A}^{0} \tau} f_{0}\right\|_{L_{2}\left(\mathbb{R}^{d}\right) \rightarrow H^{s}\left(\mathbb{R}^{d}\right)} \leq \check{C}_{s} \tau^{-1 / 2}, \quad \tau \geq 1
$$

The constant $\check{C}_{s}$ is defined by $\check{C}_{s}=\tilde{c}_{s} \alpha_{1}^{1 / 2} c_{*}^{-1 / 2}\left(1+c_{*}^{-1}\right)^{s / 2}\|f\|_{L_{\infty}}^{2}$, and depends only on $s, \alpha_{0}$, $\alpha_{1},\left\|g^{-1}\right\|_{L_{\infty}},\|f\|_{L_{\infty}},\left\|f^{-1}\right\|_{L_{\infty}}$.

Let $s$ satisfy the assumptions of Proposition 11.3; for $d=2$ we fix $s$ (for instance, $s=1 / 2$ ). Propositions 11.3 and 12.2 together with relation (12.7) imply that

$$
\varepsilon\left\|\Lambda^{\varepsilon} b(\mathbf{D}) f_{0} e^{-\mathcal{A}^{0} \tau} f_{0}\right\|_{L_{2}\left(\mathbb{R}^{d}\right) \rightarrow L_{2}\left(\mathbb{R}^{d}\right)} \leq \check{C}_{s} \widetilde{\mathfrak{C}}_{d} \tau^{-1 / 2} \varepsilon, \quad \tau>0, \quad 0<\varepsilon \leq \tau^{1 / 2} .
$$

The next result follows directly from (12.2), (12.6), and (12.8).

Theorem 12.3. Suppose that the assumptions of Theorem 12.1 are satisfied. Then we have

$$
\begin{array}{r}
\left\|f^{\varepsilon} e^{-\mathcal{A}_{\varepsilon} \tau}\left(f^{\varepsilon}\right)^{*}-\left(I+\varepsilon \Lambda^{\varepsilon} b(\mathbf{D})\right) f_{0} e^{-\mathcal{A}^{0} \tau} f_{0}\right\|_{L_{2}\left(\mathbb{R}^{d}\right) \rightarrow H^{1}\left(\mathbb{R}^{d}\right)} \leq \varepsilon\left(\mathcal{C}_{12}^{(1)} \tau^{-1}+\mathcal{C}_{12}^{(2)} \tau^{-1 / 2}\right), \\
\tau>0, \quad 0<\varepsilon \leq \tau^{1 / 2}
\end{array}
$$

The constants $\mathcal{C}_{12}^{(1)}=\left(\widehat{c}_{*}\right)^{-1 / 2} \mathcal{C}_{5}^{\prime}, \mathcal{C}_{12}^{(2)}=\mathcal{C}_{0}+\check{C}_{s} \widetilde{\mathfrak{C}}_{d}$ depend only on $d, m, n, \alpha_{0}, \alpha_{1},\|g\|_{L_{\infty}}$, $\left\|g^{-1}\right\|_{L_{\infty}},\|f\|_{L_{\infty}},\left\|f^{-1}\right\|_{L_{\infty}}$, and the parameters of the lattice $\Gamma$.

\subsection{Approximation of the operator $g^{\varepsilon} b(\mathbf{D}) f^{\varepsilon} e^{-\mathcal{A}_{\varepsilon} \tau}\left(f^{\varepsilon}\right)^{*}$}

By the scaling transformation, Theorem 10.4 implies the following statement.

Theorem 12.4. Suppose that the assumptions of Theorem 12.1 are satisfied. Let $\widetilde{g}(\mathbf{x})$ be the matrix (6.12), and let $\widetilde{g}^{\varepsilon}(\mathbf{x})=\widetilde{g}\left(\varepsilon^{-1} \mathbf{x}\right)$. Then for $\tau>0$ and $\varepsilon>0$ we have

$$
\begin{gathered}
\left\|g^{\varepsilon} b(\mathbf{D}) f^{\varepsilon} e^{-\mathcal{A}_{\varepsilon} \tau}\left(f^{\varepsilon}\right)^{*}-\widetilde{g}^{\varepsilon} b(\mathbf{D}) f_{0} e^{-\mathcal{A}^{0} \tau} f_{0} \Pi_{\varepsilon}\right\|_{L_{2}\left(\mathbb{R}^{d}\right) \rightarrow L_{2}\left(\mathbb{R}^{d}\right)} \leq \Sigma_{2}(\tau, \varepsilon), \\
\Sigma_{2}(\tau, \varepsilon)=\left\{\begin{array}{c}
\mathcal{C}_{7} \tau^{-1} \varepsilon, \tau>0, \quad 0<\varepsilon \leq \tau^{1 / 2} \\
\mathcal{C}_{8} \tau^{-1 / 2}, \quad \tau>0, \quad \varepsilon>\tau^{1 / 2}
\end{array}\right.
\end{gathered}
$$

The constants $\mathcal{C}_{7}, \mathcal{C}_{8}$ depend only on $d, m, \alpha_{0}, \alpha_{1},\|g\|_{L_{\infty}},\left\|g^{-1}\right\|_{L_{\infty}},\|f\|_{L_{\infty}},\left\|f^{-1}\right\|_{L_{\infty}}$, and the parameters of the lattice $\Gamma$.

Similarly, the following result is deduced from Theorem 10.5.

Theorem 12.5. Under the assumptions of Theorem 12.4, we have

$$
\left\|g^{\varepsilon} b(\mathbf{D}) f^{\varepsilon} e^{-\mathcal{A}_{\varepsilon} \tau}\left(f^{\varepsilon}\right)^{*}-\widetilde{g}^{\varepsilon} b(\mathbf{D}) f_{0} e^{-\mathcal{A}^{0} \tau} f_{0}\right\|_{L_{2}\left(\mathbb{R}^{d}\right) \rightarrow L_{2}\left(\mathbb{R}^{d}\right)} \leq \mathcal{C}_{7}^{\prime} \tau^{-1} \varepsilon, \quad \tau>0, \quad 0<\varepsilon \leq \tau^{1 / 2} .
$$

The constant $\mathcal{C}_{7}^{\prime}$ depends only on $d, m, n, \alpha_{0}, \alpha_{1},\|g\|_{L_{\infty}},\left\|g^{-1}\right\|_{L_{\infty}},\|f\|_{L_{\infty}},\left\|f^{-1}\right\|_{L_{\infty}}$, and the parameters of the lattice $\Gamma$. 


\subsection{Special cases}

Suppose that condition (7.41) is satisfied. Then, by the scaling transformation, (10.2) implies that

$$
\begin{gathered}
\left\|\widehat{\mathcal{A}}_{\varepsilon}^{1 / 2}\left(f^{\varepsilon} e^{-\mathcal{A}_{\varepsilon} \tau}\left(f^{\varepsilon}\right)^{*}-f_{0} e^{-\mathcal{A}^{0} \tau} f_{0}\right)\right\|_{L_{2}\left(\mathbb{R}^{d}\right) \rightarrow L_{2}\left(\mathbb{R}^{d}\right)} \leq \widetilde{\Psi}_{2}^{0}(\tau, \varepsilon), \quad \tau>0, \quad \varepsilon>0 \\
\widetilde{\Psi}_{2}^{0}(\tau, \varepsilon)=\left\{\begin{array}{c}
\mathcal{C}_{5}^{0} \tau^{-1} \varepsilon, \tau>0, \quad 0<\varepsilon \leq \tau^{1 / 2} \\
\mathcal{C}_{6}^{0} \tau^{-1 / 2}, \quad \tau>0, \quad \varepsilon>\tau^{1 / 2}
\end{array}\right.
\end{gathered}
$$

Combining this with (11.4), we obtain

$$
\left\|\mathbf{D}\left(f^{\varepsilon} e^{-\mathcal{A}_{\varepsilon} \tau}\left(f^{\varepsilon}\right)^{*}-f_{0} e^{-\mathcal{A}^{0} \tau} f_{0}\right)\right\|_{L_{2}\left(\mathbb{R}^{d}\right) \rightarrow L_{2}\left(\mathbb{R}^{d}\right)} \leq\left(\widehat{c}_{*}\right)^{-1 / 2} \widetilde{\Psi}_{2}^{0}(\tau, \varepsilon), \quad \tau>0, \quad \varepsilon>0 .
$$

Together with (12.2) this yields the following result.

Proposition 12.6. Suppose that the assumptions of Theorem 12.1 and also condition (7.41) are satisfied. Then for $\tau>0$ and $\varepsilon>0$ we have

$$
\begin{aligned}
& \left\|f^{\varepsilon} e^{-\mathcal{A}_{\varepsilon} \tau}\left(f^{\varepsilon}\right)^{*}-f_{0} e^{-\mathcal{A}^{0} \tau} f_{0}\right\|_{L_{2}\left(\mathbb{R}^{d}\right) \rightarrow H^{1}\left(\mathbb{R}^{d}\right)} \leq \Psi_{2}^{0}(\tau, \varepsilon), \\
& \Psi_{2}^{0}(\tau, \varepsilon)=\left\{\begin{aligned}
\varepsilon\left(\check{\mathcal{C}}_{5}^{0} \tau^{-1}+\mathcal{C}_{0} \tau^{-1 / 2}\right), & \tau>0, \quad 0<\varepsilon \leq \tau^{1 / 2} \\
\tau^{-1 / 2}\left(\check{\mathcal{C}}_{6}^{0}+\mathcal{C}_{0} \varepsilon\right), & \tau>0, \quad \varepsilon>\tau^{1 / 2}
\end{aligned}\right.
\end{aligned}
$$

The constants $\check{\mathcal{C}}_{5}^{0}=\left(\widehat{c}_{*}\right)^{-1 / 2} \mathcal{C}_{5}^{0}, \check{\mathcal{C}}_{6}^{0}=\left(\widehat{c}_{*}\right)^{-1 / 2} \mathcal{C}_{6}^{0}, \mathcal{C}_{0}$ depend only on $\alpha_{0}, \alpha_{1},\|g\|_{L_{\infty}},\left\|g^{-1}\right\|_{L_{\infty}}$, $\|f\|_{L_{\infty}},\left\|f^{-1}\right\|_{L_{\infty}}$, and the parameters of the lattice $\Gamma$.

Finally, by the scaling transformation, Proposition 10.7 implies the following statement.

Proposition 12.7. Suppose that the assumptions of Theorem 12.1 and also condition (6.20) are satisfied. Then for $\tau>0$ and $\varepsilon>0$ we have

$$
\begin{gathered}
\left\|g^{\varepsilon} b(\mathbf{D}) f^{\varepsilon} e^{-\mathcal{A}_{\varepsilon} \tau}\left(f^{\varepsilon}\right)^{*}-g^{0} b(\mathbf{D}) f_{0} e^{-\mathcal{A}^{0} \tau} f_{0}\right\|_{L_{2}\left(\mathbb{R}^{d}\right) \rightarrow L_{2}\left(\mathbb{R}^{d}\right)} \leq \Sigma_{2}^{0}(\tau, \varepsilon), \\
\Sigma_{2}^{0}(\tau, \varepsilon)=\left\{\begin{array}{c}
\mathcal{C}_{7}^{0} \tau^{-1} \varepsilon, \quad \tau>0, \quad 0<\varepsilon \leq \tau^{1 / 2} \\
\mathcal{C}_{8}^{0} \tau^{-1 / 2}, \quad \tau>0, \quad \varepsilon>\tau^{1 / 2}
\end{array}\right.
\end{gathered}
$$

The constant $\mathcal{C}_{8}^{0}$ depends only on $\alpha_{0}, \alpha_{1},\|g\|_{L_{\infty}},\left\|g^{-1}\right\|_{L_{\infty}},\|f\|_{L_{\infty}},\left\|f^{-1}\right\|_{L_{\infty}} ;$ the constant $\mathcal{C}_{7}^{0}$ depends on the same parameters and on $d, m$, and the parameters of the lattice $\Gamma$.

\section{Homogenization of a parabolic Cauchy problem}

\subsection{The homogeneous Cauchy problem}

Now we apply the results of Sec. 11, 12 to investigate the behavior of the solutions of a parabolic Cauchy problem in the small period limit. 
Let $\mathbf{u}_{\varepsilon}(\mathbf{x}, \tau), \mathbf{x} \in \mathbb{R}^{d}, \tau \geq 0$, be the solution of the problem

$$
\frac{\partial \mathbf{u}_{\varepsilon}}{\partial \tau}=-b(\mathbf{D})^{*} g^{\varepsilon}(\mathbf{x}) b(\mathbf{D}) \mathbf{u}_{\varepsilon}, \quad \mathbf{u}_{\varepsilon}(\mathbf{x}, 0)=\phi(\mathbf{x})
$$

where $\phi \in L_{2}\left(\mathbb{R}^{d} ; \mathbb{C}^{n}\right)$. The solution $\mathbf{u}_{\varepsilon}$ can be represented in terms of the operator exponential: $\mathbf{u}_{\varepsilon}=\exp \left(-\widehat{\mathcal{A}}_{\varepsilon} \tau\right) \phi$.

Let $\mathbf{u}_{0}$ be the solution of the "homogenized" problem

$$
\frac{\partial \mathbf{u}_{0}}{\partial \tau}=-b(\mathbf{D})^{*} g^{0} b(\mathbf{D}) \mathbf{u}_{0}, \quad \mathbf{u}_{0}(\mathbf{x}, 0)=\phi(\mathbf{x}),
$$

where $g^{0}$ is the effective matrix defined by (6.13). Then $\mathbf{u}_{0}=\exp \left(-\widehat{\mathcal{A}}^{0} \tau\right) \boldsymbol{\phi}$.

We obtain approximation of the solution $\mathbf{u}_{\varepsilon}$ in the norm of $H^{1}\left(\mathbb{R}^{d} ; \mathbb{C}^{n}\right)$ and approximation of the so called "flux" $\mathbf{p}_{\varepsilon}=g^{\varepsilon} b(\mathbf{D}) \mathbf{u}_{\varepsilon}$ in the norm of $L_{2}\left(\mathbb{R}^{d} ; \mathbb{C}^{m}\right)$, for a fixed $\tau>0$ and small $\varepsilon$. In all the statements of this section, we assume that $\Lambda(\mathbf{x})$ is the $\Gamma$-periodic solution of problem (6.11), and $\Lambda^{\varepsilon}(\mathbf{x})=\Lambda\left(\varepsilon^{-1} \mathbf{x}\right) ; \widetilde{g}(\mathbf{x})$ is the matrix (6.12), and $\widetilde{g}^{\varepsilon}(\mathbf{x})=\widetilde{g}\left(\varepsilon^{-1} \mathbf{x}\right)$.

Applying Theorems 11.4 and 11.6, we obtain the following result.

Theorem 13.1. Let $\mathbf{u}_{\varepsilon}$ be the solution of problem (13.1), and let $\mathbf{u}_{0}$ be the solution of problem (13.2). Let $\mathbf{p}_{\varepsilon}=g^{\varepsilon} b(\mathbf{D}) \mathbf{u}_{\varepsilon}$. Then for $\tau>0$ and $0<\varepsilon \leq \tau^{1 / 2}$ we have

$$
\begin{gathered}
\left\|\mathbf{u}_{\varepsilon}(\cdot, \tau)-\mathbf{u}_{0}(\cdot, \tau)-\varepsilon \Lambda^{\varepsilon} b(\mathbf{D}) \mathbf{u}_{0}(\cdot, \tau)\right\|_{H^{1}\left(\mathbb{R}^{d}\right)} \leq C(\tau) \varepsilon\|\boldsymbol{\phi}\|_{L_{2}\left(\mathbb{R}^{d}\right)}, \\
\left\|\mathbf{p}_{\varepsilon}(\cdot, \tau)-\widetilde{g}^{\varepsilon} b(\mathbf{D}) \mathbf{u}_{0}(\cdot, \tau)\right\|_{L_{2}\left(\mathbb{R}^{d}\right)} \leq \mathcal{C}_{3}^{\prime} \tau^{-1} \varepsilon\|\boldsymbol{\phi}\|_{L_{2}\left(\mathbb{R}^{d}\right)} .
\end{gathered}
$$

Here $C(\tau)=\mathcal{C}_{10}^{(1)} \tau^{-1}+\mathcal{C}_{10}^{(2)} \tau^{-1 / 2}$. The constants $\mathcal{C}_{10}^{(1)}, \mathcal{C}_{10}^{(2)}, \mathcal{C}_{3}^{\prime}$ depend only on $d, m, n, \alpha_{0}$, $\alpha_{1},\|g\|_{L_{\infty}},\left\|g^{-1}\right\|_{L_{\infty}}$, and the parameters of the lattice $\Gamma$.

\subsection{The nonhomogeneous Cauchy problem}

Now we consider the following Cauchy problem for a nonhomogeneous equation:

$$
\begin{aligned}
& \frac{\partial \mathbf{u}_{\varepsilon}}{\partial \tau}=-b(\mathbf{D})^{*} g^{\varepsilon}(\mathbf{x}) b(\mathbf{D}) \mathbf{u}_{\varepsilon}+\mathbf{F}(\mathbf{x}, \tau), \quad \mathbf{x} \in \mathbb{R}^{d}, \quad 0<\tau \leq T, \\
& \mathbf{u}_{\varepsilon}(\mathbf{x}, 0)=\boldsymbol{\phi}(\mathbf{x}) .
\end{aligned}
$$

Here $0<T \leq \infty, \phi \in L_{2}\left(\mathbb{R}^{d} ; \mathbb{C}^{n}\right), \mathbf{F} \in \mathcal{H}_{p}(T):=L_{p}\left((0, T) ; L_{2}\left(\mathbb{R}^{d} ; \mathbb{C}^{n}\right)\right), 2<p \leq \infty$. (If $T=\infty$, then $0<\tau<\infty$ in the equation.) We denote

$$
\begin{aligned}
\|\mathbf{F}\|_{\mathcal{H}_{p}(\tau)} & :=\left(\int_{0}^{\tau}\|\mathbf{F}(\cdot, \tilde{\tau})\|_{L_{2}\left(\mathbb{R}^{d}\right)}^{p} d \tilde{\tau}\right)^{1 / p}, \quad 1 \leq p<\infty, \\
\|\mathbf{F}\|_{\mathcal{H}_{\infty}(\tau)} & :=\operatorname{ess}-\sup _{0<\tilde{\tau}<\tau}\|\mathbf{F}(\cdot, \tilde{\tau})\|_{L_{2}\left(\mathbb{R}^{d}\right)} .
\end{aligned}
$$

The solution $\mathbf{u}_{\varepsilon}$ of problem (13.3) is represented as

$$
\mathbf{u}_{\varepsilon}(\cdot, \tau)=e^{-\widehat{\mathcal{A}}_{\varepsilon} \tau} \boldsymbol{\phi}+\int_{0}^{\tau} e^{-\widehat{\mathcal{A}}_{\varepsilon}(\tau-\tilde{\tau})} \mathbf{F}(\cdot, \tilde{\tau}) d \tilde{\tau} .
$$


Let $\mathbf{u}_{0}$ be the solution of the "homogenized" problem

$$
\begin{aligned}
& \frac{\partial \mathbf{u}_{0}}{\partial \tau}=-b(\mathbf{D})^{*} g^{0} b(\mathbf{D}) \mathbf{u}_{0}+\mathbf{F}(\mathbf{x}, \tau), \quad \mathbf{x} \in \mathbb{R}^{d}, \quad 0<\tau \leq T \\
& \mathbf{u}_{0}(\mathbf{x}, 0)=\boldsymbol{\phi}(\mathbf{x})
\end{aligned}
$$

Then

$$
\mathbf{u}_{0}(\cdot, \tau)=e^{-\widehat{\mathcal{A}}^{0} \tau} \boldsymbol{\phi}+\int_{0}^{\tau} e^{-\widehat{\mathcal{A}}^{0}(\tau-\tilde{\tau})} \mathbf{F}(\cdot, \tilde{\tau}) d \tilde{\tau} .
$$

Now we rely on Theorems 11.1 and 11.5 , since in order to approximate the solution $\mathbf{u}_{\varepsilon}$ in $H^{1}\left(\mathbb{R}^{d} ; \mathbb{C}^{n}\right)$ for a fixed $\tau>0$, we need to use approximations for the operator exponential $\exp \left(-\widehat{\mathcal{A}}_{\varepsilon} s\right)$ for all values $s \in(0, \tau]$.

Theorem 13.2. Let $\mathbf{u}_{\varepsilon}$ be the solution of problem (13.3), where $\boldsymbol{\phi} \in L_{2}\left(\mathbb{R}^{d} ; \mathbb{C}^{n}\right)$ and $\mathbf{F} \in$ $\mathcal{H}_{p}(T), 2<p \leq \infty$. Let $\mathbf{u}_{0}$ be the solution of problem $(13.5)$. Let $\mathbf{p}_{\varepsilon}=g^{\varepsilon} b(\mathbf{D}) \mathbf{u}_{\varepsilon}$. Let $\Pi_{\varepsilon}$ be the operator (11.1). Then for $0<\tau \leq T$ and $0<\varepsilon \leq \tau^{1 / 2}$ we have

$$
\left\|\mathbf{u}_{\varepsilon}(\cdot, \tau)-\mathbf{u}_{0}(\cdot, \tau)-\varepsilon \Lambda^{\varepsilon} b(\mathbf{D}) \Pi_{\varepsilon} \mathbf{u}_{0}(\cdot, \tau)\right\|_{H^{1}\left(\mathbb{R}^{d}\right)} \leq C_{0}(\tau) \varepsilon\|\boldsymbol{\phi}\|_{L_{2}\left(\mathbb{R}^{d}\right)}+\rho_{1}(\tau, \varepsilon ; p)\|\mathbf{F}\|_{\mathcal{H}_{p}(\tau)},
$$

where $C_{0}(\tau)=\check{\mathcal{C}}_{1} \tau^{-1}+\mathcal{C}_{9} \tau^{-1 / 2}$,

$$
\begin{aligned}
\rho_{1}(\tau, \varepsilon ; p) & =\left\{\begin{array}{c}
c_{p}^{\prime} \varepsilon^{1-2 / p}+c_{p}^{\prime \prime} \varepsilon \tau^{1 / 2-1 / p}, \quad 2<p<\infty, \\
\check{\mathcal{C}}_{1} \varepsilon \ln \left(\tau \varepsilon^{-2}\right)+2 \check{\mathcal{C}}_{2} \varepsilon+2 \mathcal{C}_{9} \varepsilon \tau^{1 / 2}, \quad p=\infty,
\end{array}\right. \\
c_{p}^{\prime} & =\check{\mathcal{C}}_{1}\left(p^{\prime}-1\right)^{-1 / p^{\prime}}+\check{\mathcal{C}}_{2}\left(1-p^{\prime} / 2\right)^{-1 / p^{\prime}}, \quad c_{p}^{\prime \prime}=\mathcal{C}_{9}\left(1-p^{\prime} / 2\right)^{-1 / p^{\prime}} .
\end{aligned}
$$

Here $p^{-1}+\left(p^{\prime}\right)^{-1}=1$. For $0<\tau \leq T$ and $0<\varepsilon \leq \tau^{1 / 2}$ we have

$$
\begin{gathered}
\left\|\mathbf{p}_{\varepsilon}(\cdot, \tau)-\widetilde{g}^{\varepsilon} b(\mathbf{D}) \Pi_{\varepsilon} \mathbf{u}_{0}(\cdot, \tau)\right\|_{L_{2}\left(\mathbb{R}^{d}\right)} \leq \mathcal{C}_{3} \tau^{-1} \varepsilon\|\phi\|_{L_{2}\left(\mathbb{R}^{d}\right)}+\rho_{2}(\tau, \varepsilon ; p)\|\mathbf{F}\|_{\mathcal{H}_{p}(\tau)}, \\
\rho_{2}(\tau, \varepsilon ; p)=\left\{\begin{array}{c}
\check{c}_{p} \varepsilon^{1-2 / p}, \quad 2<p<\infty, \\
\mathcal{C}_{3} \varepsilon \ln \left(\tau \varepsilon^{-2}\right)+2 \mathcal{C}_{4} \varepsilon, \quad p=\infty, \\
\check{c}_{p}=\mathcal{C}_{3}\left(p^{\prime}-1\right)^{-1 / p^{\prime}}+\mathcal{C}_{4}\left(1-p^{\prime} / 2\right)^{-1 / p^{\prime}}
\end{array}\right.
\end{gathered}
$$

Proof. Let $0<\tau \leq T$ and $0<\varepsilon \leq \tau^{1 / 2}$. By (11.12), (13.4), (13.6), we have

$$
\begin{aligned}
& \left\|\mathbf{u}_{\varepsilon}(\cdot, \tau)-\mathbf{u}_{0}(\cdot, \tau)-\varepsilon \Lambda^{\varepsilon} b(\mathbf{D}) \Pi_{\varepsilon} \mathbf{u}_{0}(\cdot, \tau)\right\|_{H^{1}\left(\mathbb{R}^{d}\right)} \\
& \quad \leq C_{0}(\tau) \varepsilon\|\phi\|_{L_{2}\left(\mathbb{R}^{d}\right)}+\int_{0}^{\tau} \Psi_{1}(\tau-\tilde{\tau}, \varepsilon)\|\mathbf{F}(\cdot, \tilde{\tau})\|_{L_{2}\left(\mathbb{R}^{d}\right)} d \tilde{\tau}
\end{aligned}
$$

where $C_{0}(\tau)=\check{\mathcal{C}}_{1} \tau^{-1}+\mathcal{C}_{9} \tau^{-1 / 2}$, and $\Psi_{1}(\tau, \varepsilon)$ is defined by (11.13). The integral in the right-hand side of (13.10) can be written as

$$
\begin{aligned}
& \int_{0}^{\tau} \Psi_{1}(\tau-\tilde{\tau}, \varepsilon)\|\mathbf{F}(\cdot, \tilde{\tau})\|_{L_{2}\left(\mathbb{R}^{d}\right)} d \tilde{\tau}=\check{\mathcal{C}}_{1} \varepsilon \int_{0}^{\tau-\varepsilon^{2}}(\tau-\tilde{\tau})^{-1}\|\mathbf{F}(\cdot, \tilde{\tau})\|_{L_{2}\left(\mathbb{R}^{d}\right)} d \tilde{\tau} \\
& +\check{\mathcal{C}}_{2} \int_{\tau-\varepsilon^{2}}^{\tau}(\tau-\tilde{\tau})^{-1 / 2}\|\mathbf{F}(\cdot, \tilde{\tau})\|_{L_{2}\left(\mathbb{R}^{d}\right)} d \tilde{\tau}+\mathcal{C}_{9} \varepsilon \int_{0}^{\tau}(\tau-\tilde{\tau})^{-1 / 2}\|\mathbf{F}(\cdot, \tilde{\tau})\|_{L_{2}\left(\mathbb{R}^{d}\right)} d \tilde{\tau}
\end{aligned}
$$


For $2<p<\infty$ we apply the Hölder inequality. Hence,

$$
\begin{aligned}
& \int_{0}^{\tau} \Psi_{1}(\tau-\tilde{\tau}, \varepsilon)\|\mathbf{F}(\cdot, \tilde{\tau})\|_{L_{2}\left(\mathbb{R}^{d}\right)} d \tilde{\tau} \leq\|\mathbf{F}\|_{\mathcal{H}_{p}(\tau)} \check{\mathcal{C}}_{1} \varepsilon\left(\int_{0}^{\tau-\varepsilon^{2}}(\tau-\tilde{\tau})^{-p^{\prime}} d \tilde{\tau}\right)^{1 / p^{\prime}} \\
& +\|\mathbf{F}\|_{\mathcal{H}_{p}(\tau)} \check{\mathcal{C}}_{2}\left(\int_{\tau-\varepsilon^{2}}^{\tau}(\tau-\tilde{\tau})^{-p^{\prime} / 2} d \tilde{\tau}\right)^{1 / p^{\prime}}+\|\mathbf{F}\|_{\mathcal{H}_{p}(\tau)} \mathcal{C}_{9} \varepsilon\left(\int_{0}^{\tau}(\tau-\tilde{\tau})^{-p^{\prime} / 2} d \tilde{\tau}\right)^{1 / p^{\prime}} \\
& \leq\|\mathbf{F}\|_{\mathcal{H}_{p}(\tau)}\left(\check{\mathcal{C}}_{1}\left(p^{\prime}-1\right)^{-1 / p^{\prime}} \varepsilon^{1-2 / p}+\check{\mathcal{C}}_{2}\left(1-p^{\prime} / 2\right)^{-1 / p^{\prime}} \varepsilon^{1-2 / p}+\mathcal{C}_{9} \varepsilon\left(1-p^{\prime} / 2\right)^{-1 / p^{\prime}} \tau^{1 / 2-1 / p}\right) .
\end{aligned}
$$

Convergence of all the integrals under consideration is ensured by the condition $2<p<\infty$, whence $1<p^{\prime}<2$. Together with (13.10) this implies (13.7) in the case where $2<p<\infty$.

If $p=\infty,(13.11)$ implies the estimate

$$
\begin{aligned}
& \int_{0}^{\tau} \Psi_{1}(\tau-\tilde{\tau}, \varepsilon)\|\mathbf{F}(\cdot, \tilde{\tau})\|_{L_{2}\left(\mathbb{R}^{d}\right)} d \tilde{\tau} \\
& \leq\|\mathbf{F}\|_{\mathcal{H}_{\infty}(\tau)}\left(\check{\mathcal{C}_{1} \varepsilon} \int_{0}^{\tau-\varepsilon^{2}}(\tau-\tilde{\tau})^{-1} d \tilde{\tau}+\check{\mathcal{C}}_{2} \int_{\tau-\varepsilon^{2}}^{\tau}(\tau-\tilde{\tau})^{-1 / 2} d \tilde{\tau}+\mathcal{C}_{9} \varepsilon \int_{0}^{\tau}(\tau-\tilde{\tau})^{-1 / 2} d \tilde{\tau}\right) \\
& =\|\mathbf{F}\|_{\mathcal{H}_{\infty}(\tau)}\left(\check{\mathcal{C}}_{1} \varepsilon \ln \left(\tau \varepsilon^{-2}\right)+2 \check{\mathcal{C}}_{2} \varepsilon+2 \mathcal{C}_{9} \varepsilon \tau^{1 / 2}\right) .
\end{aligned}
$$

Combining this with (13.10), we arrive at (13.7) in the case $p=\infty$.

In order to prove (13.9), we use (11.22), (13.4), (13.6). Then

$$
\left\|\mathbf{p}_{\varepsilon}(\cdot, \tau)-\widetilde{g}^{\varepsilon} b(\mathbf{D}) \Pi_{\varepsilon} \mathbf{u}_{0}(\cdot, \tau)\right\|_{L_{2}\left(\mathbb{R}^{d}\right)} \leq \mathcal{C}_{3} \tau^{-1} \varepsilon\|\phi\|_{L_{2}\left(\mathbb{R}^{d}\right)}+\int_{0}^{\tau} \Sigma_{1}(\tau-\tilde{\tau}, \varepsilon)\|\mathbf{F}(\cdot, \tilde{\tau})\|_{L_{2}\left(\mathbb{R}^{d}\right)} d \tilde{\tau} .
$$

Using (11.23), we rewrite the integral in (13.12) as

$$
\begin{aligned}
& \int_{0}^{\tau} \Sigma_{1}(\tau-\tilde{\tau}, \varepsilon)\|\mathbf{F}(\cdot, \tilde{\tau})\|_{L_{2}\left(\mathbb{R}^{d}\right)} d \tilde{\tau} \\
& =\mathcal{C}_{3} \varepsilon \int_{0}^{\tau-\varepsilon^{2}}(\tau-\tilde{\tau})^{-1}\|\mathbf{F}(\cdot, \tilde{\tau})\|_{L_{2}\left(\mathbb{R}^{d}\right)} d \tilde{\tau}+\mathcal{C}_{4} \int_{\tau-\varepsilon^{2}}^{\tau}(\tau-\tilde{\tau})^{-1 / 2}\|\mathbf{F}(\cdot, \tilde{\tau})\|_{L_{2}\left(\mathbb{R}^{d}\right)} d \tilde{\tau}
\end{aligned}
$$

If $2<p<\infty$, by the Hölder inequality, we obtain

$$
\int_{0}^{\tau} \Sigma_{1}(\tau-\tilde{\tau}, \varepsilon)\|\mathbf{F}(\cdot, \tilde{\tau})\|_{L_{2}\left(\mathbb{R}^{d}\right)} d \tilde{\tau} \leq\|\mathbf{F}\|_{\mathcal{H}_{p}(\tau)}\left(\mathcal{C}_{3}\left(p^{\prime}-1\right)^{-1 / p^{\prime}}+\mathcal{C}_{4}\left(1-p^{\prime} / 2\right)^{-1 / p^{\prime}}\right) \varepsilon^{1-2 / p}
$$

Together with (13.12) this proves (13.9) in the case $2<p<\infty$.

If $p=\infty$, it follows from (13.13) that

$$
\int_{0}^{\tau} \Sigma_{1}(\tau-\tilde{\tau}, \varepsilon)\|\mathbf{F}(\cdot, \tilde{\tau})\|_{L_{2}\left(\mathbb{R}^{d}\right)} d \tilde{\tau} \leq\|\mathbf{F}\|_{\mathcal{H}_{\infty}(\tau)}\left(\mathcal{C}_{3} \varepsilon \ln \left(\tau \varepsilon^{-2}\right)+2 \mathcal{C}_{4} \varepsilon\right) .
$$

Together with (13.12), this yields (13.9) if $p=\infty$. 


\subsection{Special cases for problem (13.3)}

The case where the corrector in (13.7) is equal to zero is considered with the help of Proposition 11.7. It is easy to deduce the following statement by analogy with the proof of inequality (13.7).

Proposition 13.3. Suppose that the assumptions of Theorem 13.2 and also condition (7.41) are satisfied. Then for $0<\tau \leq T$ and $0<\varepsilon \leq \tau^{1 / 2}$ we have

$$
\left\|\mathbf{u}_{\varepsilon}(\cdot, \tau)-\mathbf{u}_{0}(\cdot, \tau)\right\|_{H^{1}\left(\mathbb{R}^{d}\right)} \leq C_{*}(\tau) \varepsilon\|\phi\|_{L_{2}\left(\mathbb{R}^{d}\right)}+\rho_{3}(\tau, \varepsilon ; p)\|\mathbf{F}\|_{\mathcal{H}_{p}(\tau)},
$$

where $C_{*}(\tau)=\check{\mathcal{C}}_{1}^{0} \tau^{-1}+\widehat{\mathcal{C}}_{0} \tau^{-1 / 2}$,

$$
\begin{aligned}
\rho_{3}(\tau, \varepsilon ; p) & =\left\{\begin{array}{c}
\tilde{c}_{p}^{\prime} \varepsilon \varepsilon^{1-2 / p}+\tilde{c}_{p}^{\prime \prime} \varepsilon \tau^{1 / 2-1 / p}, \quad 2<p<\infty, \\
\check{\mathcal{C}}_{1}^{0} \varepsilon \ln \left(\tau \varepsilon^{-2}\right)+2 \check{\mathcal{C}}_{2}^{0} \varepsilon+2 \widehat{\mathcal{C}}_{0} \varepsilon \tau^{1 / 2}, \quad p=\infty,
\end{array}\right. \\
\tilde{c}_{p}^{\prime} & =\check{\mathcal{C}}_{1}^{0}\left(p^{\prime}-1\right)^{-1 / p^{\prime}}+\check{\mathcal{C}}_{2}^{0}\left(1-p^{\prime} / 2\right)^{-1 / p^{\prime}}, \quad \tilde{c}_{p}^{\prime \prime}=\widehat{\mathcal{C}}_{0}\left(1-p^{\prime} / 2\right)^{-1 / p^{\prime}} .
\end{aligned}
$$

Now we distinguish the special case where approximation of the flux $\mathbf{p}_{\varepsilon}$ does not depend on $\varepsilon$. Using Proposition 11.8, it is easy to check the following statement by analogy with the proof of inequality (13.9).

Proposition 13.4. Suppose that the assumptions of Theorem 13.2 and also condition (6.20) are satisfied. Then for $0<\tau \leq T$ and $0<\varepsilon \leq \tau^{1 / 2}$ we have

$$
\left\|\mathbf{p}_{\varepsilon}(\cdot, \tau)-g^{0} b(\mathbf{D}) \mathbf{u}_{0}(\cdot, \tau)\right\|_{L_{2}\left(\mathbb{R}^{d}\right)} \leq \mathcal{C}_{3}^{0} \tau^{-1} \varepsilon\|\phi\|_{L_{2}\left(\mathbb{R}^{d}\right)}+\rho_{4}(\tau, \varepsilon ; p)\|\mathbf{F}\|_{\mathcal{H}_{p}(\tau)},
$$

where

$$
\begin{aligned}
\rho_{4}(\tau, \varepsilon ; p) & =\left\{\begin{array}{c}
c_{p}^{0} \varepsilon^{1-2 / p}, \quad 2<p<\infty, \\
\mathcal{C}_{3}^{0} \varepsilon \ln \left(\tau \varepsilon^{-2}\right)+2 \mathcal{C}_{4}^{0} \varepsilon, \quad p=\infty,
\end{array}\right. \\
c_{p}^{0} & =\mathcal{C}_{3}^{0}\left(p^{\prime}-1\right)^{-1 / p^{\prime}}+\mathcal{C}_{4}^{0}\left(1-p^{\prime} / 2\right)^{-1 / p^{\prime}}
\end{aligned}
$$

\subsection{More general homogeneous Cauchy problem}

Now we consider more general Cauchy problem. We start with the case of homogeneous equation. Let $Q(\mathbf{x})$ be a measurable $\Gamma$-periodic $(n \times n)$-matrix-valued function in $\mathbb{R}^{d}$ such that $Q, Q^{-1} \in L_{\infty}$ and $Q(\mathbf{x})>0$. Let $\mathbf{v}_{\varepsilon}(\mathbf{x}, \tau), \mathbf{x} \in \mathbb{R}^{d}, \tau \geq 0$, be the solution of the problem

$$
Q^{\varepsilon}(\mathbf{x}) \frac{\partial \mathbf{v}_{\varepsilon}}{\partial \tau}=-b(\mathbf{D})^{*} g^{\varepsilon}(\mathbf{x}) b(\mathbf{D}) \mathbf{v}_{\varepsilon}, \quad Q^{\varepsilon}(\mathbf{x}) \mathbf{v}_{\varepsilon}(\mathbf{x}, 0)=\phi(\mathbf{x})
$$

where $\boldsymbol{\phi} \in L_{2}\left(\mathbb{R}^{d} ; \mathbb{C}^{n}\right)$. We write $Q(\mathbf{x})^{-1}$ in a factorized form $Q(\mathbf{x})^{-1}=f(\mathbf{x}) f(\mathbf{x})^{*}$, where $f(\mathbf{x})$ is a $\Gamma$-periodic $(n \times n)$-matrix-valued function such that $f, f^{-1} \in L_{\infty}$. (For instance, 
one can put $f(\mathbf{x})=Q(\mathbf{x})^{-1 / 2}$.) We put $\mathbf{w}_{\varepsilon}=\left(f^{\varepsilon}\right)^{-1} \mathbf{v}_{\varepsilon}$. Then $\mathbf{w}_{\varepsilon}$ is the solution of the problem

$$
\frac{\partial \mathbf{w}_{\varepsilon}}{\partial \tau}=-\left(f^{\varepsilon}(\mathbf{x})\right)^{*} b(\mathbf{D})^{*} g^{\varepsilon}(\mathbf{x}) b(\mathbf{D}) f^{\varepsilon}(\mathbf{x}) \mathbf{w}_{\varepsilon}, \quad \mathbf{w}_{\varepsilon}(\mathbf{x}, 0)=\left(f^{\varepsilon}(\mathbf{x})\right)^{*} \boldsymbol{\phi}(\mathbf{x}) .
$$

The solution of problem (13.15) is represented as $\mathbf{w}_{\varepsilon}=e^{-\mathcal{A}_{\varepsilon} \tau}\left(f^{\varepsilon}\right)^{*} \boldsymbol{\phi}$. Then

$$
\mathbf{v}_{\varepsilon}=f^{\varepsilon} e^{-\mathcal{A}_{\varepsilon} \tau}\left(f^{\varepsilon}\right)^{*} \phi
$$

Let $\mathbf{v}_{0}$ be the solution of the "homogenized" problem

$$
\bar{Q} \frac{\partial \mathbf{v}_{0}}{\partial \tau}=-b(\mathbf{D})^{*} g^{0} b(\mathbf{D}) \mathbf{v}_{0}, \quad \bar{Q} \mathbf{v}_{0}(\mathbf{x}, 0)=\phi(\mathbf{x})
$$

Here $\bar{Q}$ is the mean value of $Q(\mathbf{x})$ over the cell $\Omega$. Let $f_{0}$ be defined by $(8.2)$, i. e., $f_{0}=(\bar{Q})^{-1 / 2}$. Then the solution of problem (13.17) can be written as

$$
\mathbf{v}_{0}=f_{0} e^{-\mathcal{A}^{0} \tau} f_{0} \phi
$$

Approximations of the solution of problem (13.14) and the corresponding flux follow directly from Theorems 12.3, 12.5 and representations (13.16), (13.18).

Theorem 13.5. Let $\mathbf{v}_{\varepsilon}$ be the solution of problem (13.14), and let $\mathbf{v}_{0}$ be the solution of problem (13.17). Let $\mathbf{q}_{\varepsilon}=g^{\varepsilon} b(\mathbf{D}) \mathbf{v}_{\varepsilon}$. Then for $\tau>0$ and $0<\varepsilon \leq \tau^{1 / 2}$ we have

$$
\begin{gathered}
\left\|\mathbf{v}_{\varepsilon}(\cdot, \tau)-\mathbf{v}_{0}(\cdot, \tau)-\varepsilon \Lambda^{\varepsilon} b(\mathbf{D}) \mathbf{v}_{0}(\cdot, \tau)\right\|_{H^{1}\left(\mathbb{R}^{d}\right)} \leq \widetilde{C}(\tau) \varepsilon\|\phi\|_{L_{2}\left(\mathbb{R}^{d}\right)} \\
\left\|\mathbf{q}_{\varepsilon}(\cdot, \tau)-\widetilde{g}^{\varepsilon} b(\mathbf{D}) \mathbf{v}_{0}(\cdot, \tau)\right\|_{L_{2}\left(\mathbb{R}^{d}\right)} \leq \mathcal{C}_{7}^{\prime} \tau^{-1} \varepsilon\|\boldsymbol{\phi}\|_{L_{2}\left(\mathbb{R}^{d}\right)} .
\end{gathered}
$$

Here $\widetilde{C}(\tau)=\mathcal{C}_{12}^{(1)} \tau^{-1}+\mathcal{C}_{12}^{(2)} \tau^{-1 / 2}$. The constants $\mathcal{C}_{12}^{(1)}, \mathcal{C}_{12}^{(2)}, \mathcal{C}_{7}^{\prime}$ depend only on $d, m, n, \alpha_{0}$, $\alpha_{1},\|g\|_{L_{\infty}},\left\|g^{-1}\right\|_{L_{\infty}},\|Q\|_{L_{\infty}},\left\|Q^{-1}\right\|_{L_{\infty}}$, and the parameters of the lattice $\Gamma$.

\subsection{More general nonhomogeneous Cauchy problem}

Now we consider the Cauchy problem for a nonhomogeneous equation:

$$
\begin{aligned}
& Q^{\varepsilon}(\mathbf{x}) \frac{\partial \mathbf{v}_{\varepsilon}}{\partial \tau}=-b(\mathbf{D})^{*} g^{\varepsilon}(\mathbf{x}) b(\mathbf{D}) \mathbf{v}_{\varepsilon}+\mathbf{F}(\mathbf{x}, \tau), \quad \mathbf{x} \in \mathbb{R}^{d}, \quad 0<\tau \leq T \\
& Q^{\varepsilon}(\mathbf{x}) \mathbf{v}_{\varepsilon}(\mathbf{x}, 0)=\boldsymbol{\phi}(\mathbf{x})
\end{aligned}
$$

where $\phi \in L_{2}\left(\mathbb{R}^{d} ; \mathbb{C}^{n}\right), \mathbf{F} \in \mathcal{H}_{p}(T), 2<p \leq \infty$. The solution $\mathbf{v}_{\varepsilon}$ is represented as

$$
\mathbf{v}_{\varepsilon}=f^{\varepsilon} e^{-\mathcal{A}_{\varepsilon} \tau}\left(f^{\varepsilon}\right)^{*} \phi+\int_{0}^{\tau} f^{\varepsilon} e^{-\mathcal{A}_{\varepsilon}(\tau-\tilde{\tau})}\left(f^{\varepsilon}\right)^{*} \mathbf{F}(\cdot, \tilde{\tau}) d \tilde{\tau}
$$


Let $\mathbf{v}_{0}$ be the solution of the "homogenized" problem

$$
\begin{aligned}
& \bar{Q} \frac{\partial \mathbf{v}_{0}}{\partial \tau}=-b(\mathbf{D})^{*} g^{0} b(\mathbf{D}) \mathbf{v}_{0}+\mathbf{F}(\mathbf{x}, \tau), \quad \mathbf{x} \in \mathbb{R}^{d}, \quad 0<\tau \leq T \\
& \bar{Q} \mathbf{v}_{0}(\mathbf{x}, 0)=\boldsymbol{\phi}(\mathbf{x})
\end{aligned}
$$

The solution $\mathbf{v}_{0}$ is represented as

$$
\mathbf{v}_{0}=f_{0} e^{-\mathcal{A}^{0} \tau} f_{0} \boldsymbol{\phi}+\int_{0}^{\tau} f_{0} e^{-\mathcal{A}^{0}(\tau-\tilde{\tau})} f_{0} \mathbf{F}(\cdot, \tilde{\tau}) d \tilde{\tau}
$$

Using Theorems 12.1 and 12.4 and representations (13.20), (13.22), it is easy to prove the following result by analogy with the proof of Theorem 13.2.

Theorem 13.6. Let $\mathbf{v}_{\varepsilon}$ be the solution of problem (13.19), where $\boldsymbol{\phi} \in L_{2}\left(\mathbb{R}^{d} ; \mathbb{C}^{n}\right)$ and $\mathbf{F} \in \mathcal{H}_{p}(T), 2<p \leq \infty$. Let $\mathbf{v}_{0}$ be the solution of problem $(13.21)$. Let $\mathbf{q}_{\varepsilon}=g^{\varepsilon} b(\mathbf{D}) \mathbf{v}_{\varepsilon}$. Let $\Pi_{\varepsilon}$ be the operator (11.1). Then for $0<\tau \leq T$ and $0<\varepsilon \leq \tau^{1 / 2}$ we have

$$
\left\|\mathbf{v}_{\varepsilon}(\cdot, \tau)-\mathbf{v}_{0}(\cdot, \tau)-\varepsilon \Lambda^{\varepsilon} b(\mathbf{D}) \Pi_{\varepsilon} \mathbf{v}_{0}(\cdot, \tau)\right\|_{H^{1}\left(\mathbb{R}^{d}\right)} \leq \widetilde{C}_{0}(\tau) \varepsilon\|\phi\|_{L_{2}\left(\mathbb{R}^{d}\right)}+\rho_{5}(\tau, \varepsilon ; p)\|\mathbf{F}\|_{\mathcal{H}_{p}(\tau)},
$$

where $\widetilde{C}_{0}(\tau)=\check{\mathcal{C}}_{5} \tau^{-1}+\mathcal{C}_{11} \tau^{-1 / 2}$,

$$
\begin{aligned}
\rho_{5}(\tau, \varepsilon ; p) & =\left\{\begin{array}{c}
c_{p}^{(1)} \varepsilon^{1-2 / p}+c_{p}^{(2)} \varepsilon \tau^{1 / 2-1 / p}, \quad 2<p<\infty, \\
\check{\mathcal{C}}_{5} \varepsilon \ln \left(\tau \varepsilon^{-2}\right)+2 \check{\mathcal{C}}_{6} \varepsilon+2 \mathcal{C}_{11} \varepsilon \tau^{1 / 2}, \quad p=\infty,
\end{array}\right. \\
c_{p}^{(1)} & =\check{\mathcal{C}}_{5}\left(p^{\prime}-1\right)^{-1 / p^{\prime}}+\check{\mathcal{C}}_{6}\left(1-p^{\prime} / 2\right)^{-1 / p^{\prime}}, \quad c_{p}^{(2)}=\mathcal{C}_{11}\left(1-p^{\prime} / 2\right)^{-1 / p^{\prime}} .
\end{aligned}
$$

Here $p^{-1}+\left(p^{\prime}\right)^{-1}=1$. For $0<\tau \leq T$ and $0<\varepsilon \leq \tau^{1 / 2}$ we have

$$
\begin{gathered}
\left\|\mathbf{q}_{\varepsilon}(\cdot, \tau)-\widetilde{g}^{\varepsilon} b(\mathbf{D}) \Pi_{\varepsilon} \mathbf{v}_{0}(\cdot, \tau)\right\|_{L_{2}\left(\mathbb{R}^{d}\right)} \leq \mathcal{C}_{7} \tau^{-1} \varepsilon\|\boldsymbol{\phi}\|_{L_{2}\left(\mathbb{R}^{d}\right)}+\rho_{6}(\tau, \varepsilon ; p)\|\mathbf{F}\|_{\mathcal{H}_{p}(\tau)} \\
\rho_{6}(\tau, \varepsilon ; p)=\left\{\begin{array}{c}
c_{p}^{(3)} \varepsilon^{1-2 / p}, \quad 2<p<\infty \\
\mathcal{C}_{7} \varepsilon \ln \left(\tau \varepsilon^{-2}\right)+2 \mathcal{C}_{8} \varepsilon, \quad p=\infty
\end{array}\right. \\
c_{p}^{(3)}=\mathcal{C}_{7}\left(p^{\prime}-1\right)^{-1 / p^{\prime}}+\mathcal{C}_{8}\left(1-p^{\prime} / 2\right)^{-1 / p^{\prime}}
\end{gathered}
$$

\subsection{Special cases for problem (13.19)}

The case of zero corrector can be easily considered on the basis of Proposition 12.6.

Proposition 13.7. Suppose that the assumptions of Theorem 13.6 and also condition (7.41) are satisfied. Then for $0<\tau \leq T$ and $0<\varepsilon \leq \tau^{1 / 2}$ we have

$$
\left\|\mathbf{v}_{\varepsilon}(\cdot, \tau)-\mathbf{v}_{0}(\cdot, \tau)\right\|_{H^{1}\left(\mathbb{R}^{d}\right)} \leq \widetilde{C}_{*}(\tau) \varepsilon\|\phi\|_{L_{2}\left(\mathbb{R}^{d}\right)}+\rho_{7}(\tau, \varepsilon ; p)\|\mathbf{F}\|_{\mathcal{H}_{p}(\tau)},
$$


where $\widetilde{C}_{*}(\tau)=\check{\mathcal{C}}_{5}^{0} \tau^{-1}+\mathcal{C}_{0} \tau^{-1 / 2}$,

$$
\begin{aligned}
\rho_{7}(\tau, \varepsilon ; p) & =\left\{\begin{array}{c}
c_{p}^{(4)} \varepsilon^{1-2 / p}+c_{p}^{(5)} \varepsilon \tau^{1 / 2-1 / p}, \quad 2<p<\infty \\
\check{\mathcal{C}}_{5}^{0} \varepsilon \ln \left(\tau \varepsilon^{-2}\right)+2 \check{\mathcal{C}}_{6}^{0} \varepsilon+2 \mathcal{C}_{0} \varepsilon \tau^{1 / 2}, \quad p=\infty
\end{array}\right. \\
c_{p}^{(4)} & =\check{\mathcal{C}}_{5}^{0}\left(p^{\prime}-1\right)^{-1 / p^{\prime}}+\check{\mathcal{C}}_{6}^{0}\left(1-p^{\prime} / 2\right)^{-1 / p^{\prime}}, \quad c_{p}^{(5)}=\mathcal{C}_{0}\left(1-p^{\prime} / 2\right)^{-1 / p^{\prime}}
\end{aligned}
$$

Finally, using Proposition 12.7, we consider the case where approximation of the flux $\mathbf{q}_{\varepsilon}$ does not depend on $\varepsilon$.

Proposition 13.8. Suppose that the assumptions of Theorem 13.6 and also condition (6.20) are satisfied. Then for $0<\tau \leq T$ and $0<\varepsilon \leq \tau^{1 / 2}$ we have

$$
\left\|\mathbf{q}_{\varepsilon}(\cdot, \tau)-g^{0} b(\mathbf{D}) \mathbf{v}_{0}(\cdot, \tau)\right\|_{L_{2}\left(\mathbb{R}^{d}\right)} \leq \mathcal{C}_{7}^{0} \tau^{-1} \varepsilon\|\phi\|_{L_{2}\left(\mathbb{R}^{d}\right)}+\rho_{8}(\tau, \varepsilon ; p)\|\mathbf{F}\|_{\mathcal{H}_{p}(\tau)}
$$

where

$$
\begin{aligned}
\rho_{8}(\tau, \varepsilon ; p) & =\left\{\begin{array}{c}
c_{p}^{(6)} \varepsilon^{1-2 / p}, \quad 2<p<\infty, \\
\mathcal{C}_{7}^{0} \varepsilon \ln \left(\tau \varepsilon^{-2}\right)+2 \mathcal{C}_{8}^{0} \varepsilon, \quad p=\infty,
\end{array}\right. \\
c_{p}^{(6)} & =\mathcal{C}_{7}^{0}\left(p^{\prime}-1\right)^{-1 / p^{\prime}}+\mathcal{C}_{8}^{0}\left(1-p^{\prime} / 2\right)^{-1 / p^{\prime}}
\end{aligned}
$$

\section{References}

[BaPa] N. S. Bakhvalov, G. P. Panasenko. Homogenization: averaging processes in periodic media. Mathematical problems in the mechanics of composite materials. "Nauka", Moscow, 1984; English transl., Math. Appl. (Soviet Ser.), vol. 36, Kluwer Acad. Publishers Group, Dordrecht, 1989.

[BeLP] A. Bensoussan, J. L. Lions, G. Papanicolaou. Asymptotic analysis for periodic structures. Stud. Math. Appl., vol. 5, North-Holland Publishing Company, Amsterdam-New York, 1978, 700 pp.

[BSu1] M. Birman, T. Suslina. Threshold effects near the lower edge of the spectrum for periodic differential operators of mathematical physics. Systems, Approximation, Singular Integral Operators, and Related Topics (Bordeaux, 2000), Oper. Theory Adv. Appl., vol. 129, Birkhäuser, Basel, 2001, pp. 71-107.

[BSu2] M. Sh. Birman, T. A. Suslina. Second order periodic differential operators. Threshold properties and homogenization. Algebra i Analiz, 15 (2003), no. 5, 1-108; English transl., St. Petersburg Math. J., 15 (2004), no. 5, 639-714.

[BSu3] M. Sh. Birman, T. A. Suslina. Threshold approximations with corrector term for the resolvent of a factorized selfadjoint operator family. Algebra i Analiz, 17 (2005), no. 5, 69-90; English transl., St. Petersburg Math. J., 17 (2006), no. 5, 745-762. 
[BSu4] M. Sh. Birman, T. A. Suslina. Homogenization with corrector term for periodic elliptic differential operators, Algebra i Analiz, 17 (2005), no. 6, 1-104; English transl., St. Petersburg Math. J., 17 (2006), no. 6, 897-973.

[BSu5] M. Sh. Birman, T. A. Suslina. Homogenization with corrector for periodic differential operators. Approximation of solutions in the Sobolev class $H^{1}\left(\mathbb{R}^{d}\right)$, Algebra i Analiz, 18 (2006), no. 6, 1-130; English transl., St. Petersburg Math. J., 18 (2007), no. 6, 857-955.

[Ka] T. Kato. Perturbation theory for linear operators, 2nd ed. Grundlehren Math. Wiss., vol. 132, Springer-Verlag, Berlin-New York, 1976.

[Sa] E. Sanchez-Palencia. Nonhomogeneous media and vibration theory. Lecture Notes in Phys., vol. 127, Springer-Verlag, Berlin-New York, 1980.

[Su1] T. A. Suslina. Homogenization of periodic parabolic systems. Funktsional. Anal. i Prilozhen., 38 (2004), no. 4, 86-90; English transl., Funct. Anal. Appl., 38 (2004), no. 4, 309-312.

[Su2] T. A. Suslina. Homogenization of periodic parabolic Cauchy problem. Amer. Math. Soc. Transl., ser. 2, 220 (2007), 201-233.

[V] E. S. Vasilevskaya. A periodic parabolic Cauchy problem: homogenization with corrector. Algebra i Analiz, 20 (2009), no. 1, 3-60; English transl., St. Petersburg Math. J., 20 (2010), no. 1.

[Zh] V. V. Zhikov, On some estimates of homogenization theory. Dokl. Ros. Akad. Nauk, 406 (2006), no. 5, 597-601; English transl., Dokl. Math., 73 (2006), 96-99.

[ZhKO] V. V. Zhikov, S. M. Kozlov, O. A. Oleinik. Homogenization of differential operators. "Nauka", Moscow, 1993; English transl., Springer-Verlag, Berlin, 1994.

[ZhPas1] V. V. Zhikov, S. E. Pastukhova. On operator estimates for some problems in homogenization theory. Russ. J. Math. Phys., 12 (2005), no. 4, 515-524.

[ZhPas2] V. V. Zhikov, S. E. Pastukhova. Estimates of homogenization for a parabolic equation with periodic coefficients. Russ. J. Math. Phys., 13 (2006), no. 2, 251-265. 